\title{
An Overview on Numerical Analyses of Nematic Liquid Crystal Flows
}

\author{
S. Badia · F. Guillén-Gónzalez • \\ J.V. Gutiérrez-Santacreu
}

Published online: 27 July 2011

(C) CIMNE, Barcelona, Spain 2011

\begin{abstract}
The purpose of this work is to provide an overview of the most recent numerical developments in the field of nematic liquid crystals. The Ericksen-Leslie equations govern the motion of a nematic liquid crystal. This system, in its simplest form, consists of the Navier-Stokes equations coupled with an extra anisotropic stress tensor, which represents the effect of the nematic liquid crystal on the fluid, and a convective harmonic map equation. The sphere constraint must be enforced almost everywhere in order to obtain an energy estimate. Since an almost everywhere satisfaction of this restriction is not appropriate at a numerical level, two alternative approaches have been introduced: a penalty method and a saddle-point method. These approaches are suitable for their numerical approximation by finite elements, since a discrete version of the restriction is enough to prove the desired energy estimate.

The Ginzburg-Landau penalty function is usually used to enforce the sphere constraint. Finite element methods of
\end{abstract}

F. Guillén-Gónzalez and J.V. Gutiérrez-Santacreu work was partially supported by project MTM2009-12927, Spain.

\author{
S. Badia $(\bowtie)$ \\ International Center for Numerical Methods in Engineering \\ (CIMNE), Universitat Politècnica de Catalunya, Jordi Girona 1-3, \\ Edifici C1, 08034 Barcelona, Spain \\ e-mail: sbadia@cimne.upc.edu \\ F. Guillén-Gónzalez \\ Dpto. E.D.A.N., University of Sevilla, Aptdo. 1160, \\ 41080 Sevilla, Spain \\ e-mail: guillen@us.es \\ J.V. Gutiérrez-Santacreu \\ Dpto. de Matemática Aplicada I, University of Sevilla, \\ E.T.S.I. Informática, Avda. Reina Mercedes, 41012 Sevilla, Spain \\ e-mail: juanvi@us.es
}

mixed type will play an important role when designing numerical approximations for the penalty method in order to preserve the intrinsic energy estimate.

The inf-sup condition that makes the saddle-point method well-posed is not clear yet. The only inf-sup condition for the Lagrange multiplier is obtained in the dual space of $H^{1}(\Omega)$. But such an inf-sup condition requires more regularity for the director vector than the one provided by the energy estimate. Herein, we will present an alternative infsup condition whose proof for its discrete counterpart with finite elements is still open.

\section{Introduction}

Liquid crystals are commonly considered as the fourth state of matter, different to gases, liquids, and solids. This is due to the fact that liquid crystals exhibit phases between a liquid and a crystalline solid which are known as mesophases. There are various types of liquid crystals, according to the degree of positional or orientational ordering shown by the molecules that compose them. Different degrees of ordering can be achieved, depending on the temperature (thermotropic) and/or the concentration of a solute in a solvent (lyotropic). The simplest liquid crystal phase is the nematic one, which is made of elongated rod-like molecules with similar size, whose centers of mass have no positional order (as in an isotropic liquid), but tending to align along certain locally preferred directions, confering the anisotropic structure. The orientational order is typically modeled by a unit vector field, the director field $\boldsymbol{d}$, with $|\boldsymbol{d}|=1$, which represents the average orientation of the long axes of the molecules in a volume element at a point. As the temperature is lowered in the thermotropic case, or the concentration is increased in the lyotropic case, new thermodynamical 
states appear, and the molecules begin to separate into parallel, equally spaced layers. This is the smectic liquid crystal phase. In a smectic liquid crystal, the positions of the molecular centers of mass flow freely in each layer without correlation from one layer to the next. The angle between the director field $\boldsymbol{d}$ and the local smectic layer normal $\boldsymbol{n}$ is denoted by $\theta$. When $\theta=0$, the structure is called a smectic- $A$ liquid crystal, otherwise it is called a smectic- $C$ liquid crystal. On some occasions there are transitions from smectic-A to the smectic-C phase, i.e. the angle $\theta$ grows smoothly from zero in response to a decrease of temperature or an increase of concentration.

In the last years, the study of liquid crystals has aroused an increasing interest in biology (cell membranes), physics and engineering (in the growing technological industry of electronic devices) owing to their optical properties. The alignment of the director field $d$ is affected by applied electric or magnetic fields, that can rotate the director so that it is aligned parallel to them; the molecules of a liquid crystal exhibit dielectric or diamagnetic properties. The director field can also be affected by boundary conditions.

A usual geometry for liquid crystal display (LCD) de-

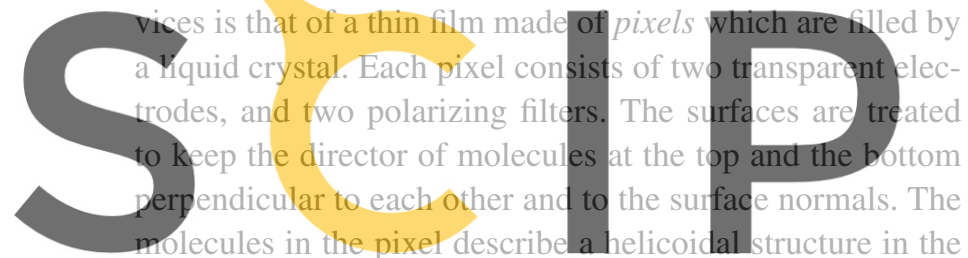
transition between surfaces. If a polarized ray of light paral-

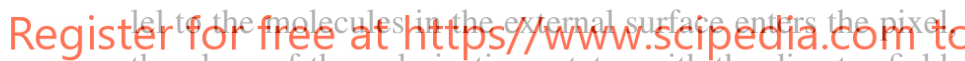
the plane of the polarization rotates with the director field. Under external electric fields normal to the surfaces, the helicoidal structure is broken, the rotation is not possible, and light is blocked by the polarizer. On the other hand, liquid crystals also provide a description of some interesting materials, as DNA or petroleum.

The two main phenomenological theories describing spatial configurations in nematic liquid crystals are the OseenFrank [21, 49] and Landau-de Gennes [16, 17] theories. Both approaches consist in modeling equilibrium states as minima of a free-energy functional. Such functionals are constructed subject to symmetry and invariance principles, to capture some properties observed from experiments.

The Oseen-Frank free energy is considered as a functional of the director vector $\boldsymbol{d}$. In its most basic form, the free energy functional is given by

$$
\begin{aligned}
E(\boldsymbol{d})= & \int_{\Omega}\left\{K_{1}|\nabla \cdot \boldsymbol{d}|^{2}+K_{2}(\boldsymbol{d} \cdot(\nabla \times \boldsymbol{d}))^{2}\right. \\
& \left.+K_{3}(\boldsymbol{d} \times(\nabla \times \boldsymbol{d}))^{2}\right\},
\end{aligned}
$$

where $K_{1}, K_{2}$, and $K_{3}$ are the splay, twist, and bend elastic constants, respectively. Note that when these constants are equal, the Dirichlet energy becomes

$E(\boldsymbol{d})=K \int_{\Omega}|\nabla \boldsymbol{d}|^{2}$.

Upon minimizing this energy subject to the sphere constraint $|\boldsymbol{d}|=1$, the following optimality system appears

$-\Delta \boldsymbol{d}-|\nabla \boldsymbol{d}|^{2} \boldsymbol{d}=0 \quad$ in $\Omega$.

The Oseen-Frank theory is limited in the sense that it can only explain point defects in liquid crystal materials but not the more complicated line and surface defects that are also observed experimentally. The defect points or singularities in liquid crystals are regions where the anisotropic properties of molecules are broken. That is, the liquid crystal behaves as an isotropic fluid. Therefore, the director field cannot be defined. Mathematically, they are modeled by $|\boldsymbol{d}|=0$. The defect points can be achieved by means of the boundaries conditions.

The Landau-de Gennes functional is established in terms of the order parameter tensor $Q$ (traceless and symmetric)
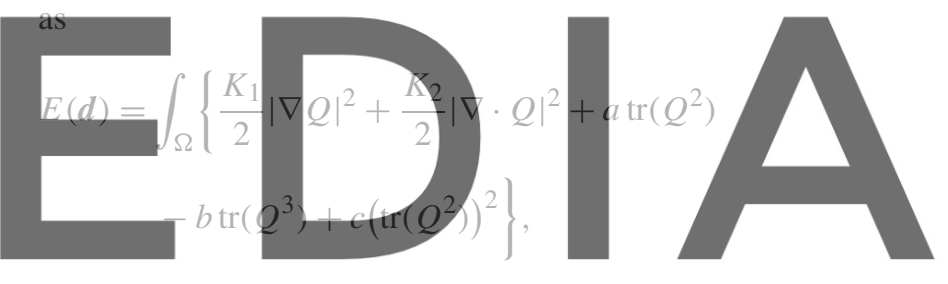

where $Q$ depends on the components of the director vector døurdoad the versilon waitholdktheitwaternark space dimension and $\operatorname{tr}(\cdot)$ indicates the trace of the matrix. This theory was one of the major achievements of P.G. de Gennes, who was awarded a Nobel prize in physics in 1991.

An alternative strategy to study the motion of defect points in liquid crystals is to consider the long-time behavior of the harmonic map flow for which it is also interesting to incorporate the influence of the velocity. On the contrary, in many situations, the anisotropic local orientation of the director field influences the stress tensors that govern the fluid velocity. The hydrodynamic theory of nematic liquid crystals was established by Ericksen [18, 19] and Leslie [35, 36]. The fundamental system consists of a set of fully coupled, macroscopic equations, that contains the Oseen-Frank elastic theory governing the steady state, equilibrium solutions. A variant of the Ericksen-Leslie equations was proposed by Lin in [37]. The equation therein is written in such a way that the sphere constraint is not explicitly enforced. The obtention of the energy law for this equation uses the fact that the director field satisfies the constraint everywhere. So, it is not suitable for numerical purposes, since the numerical approximation does not satisfy the constraint everywhere, and so, 
does not have an associated energy law. Then, some alternatives are introduced. In order to get numerical methods with an associated energy law, the sphere constraint is usually penalized with the Ginzburg-Landau penalty function such as in the works of Becker, Feng and Prohl [8], and Lin, Liu, and Zhang [41]. An alternative recently proposed by Badia, Guillén-González and Gutiérrez-Santacreu in [5] is to use an equivalent saddle-point formulation of the system proposed by Lin in [37]. It provides a system of partial differential equations equivalent to the one in [37] that also leads to numerical methods with an energy law. Furthermore, a Ginzburg-Landau-type penalization can be introduced in the saddle-point version, and treat both the original and penalized problems using a unified numerical approach.

The goal of this paper is to present the different approaches used so far for the numerical approximation of nematic liquid crystal flows. Section 2 begins with a description of the function spaces which we will draw on throughout this work. We then describe the differential approaches commented above. In Sect. 3, we will analyze the advantages and disadvantages of existing finite element approximations in the literature for the Ginzburg-Landau penalization in terms of stability and convergence. We will make

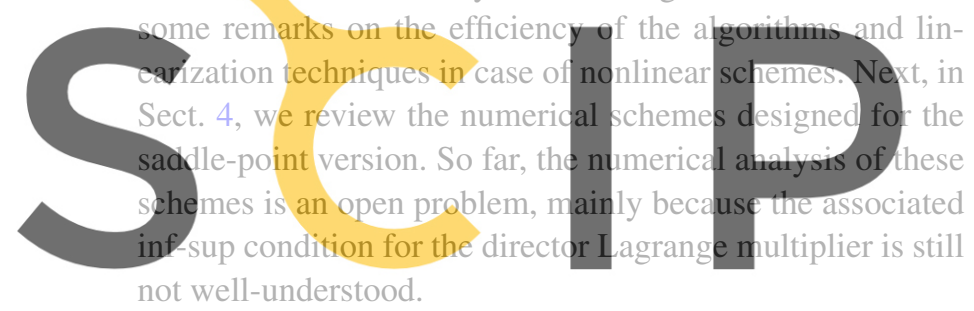

\section{Register for free at https//www.scipedia.com to}

\section{Problem Statement}

\subsection{Some Function Spaces}

We will assume the following notation throughout this paper. Let $\Omega \subset \mathbb{R}^{d}$, with $d=2$ or 3 , be a Lebesgue-measurable domain and let $1 \leq p \leq \infty$. We denote by $L^{p}(\Omega)$ the space of all Lesbegue-measurable real-valued functions, $f: \Omega \rightarrow$ $\mathbb{R}$, being $p$ th-summable in $\Omega$ for $p<\infty$ or essentially bounded for $p=\infty$, and by $\|f\|_{L^{p}(\Omega)}$ its norm. When $p=2$, the $L^{2}(\Omega)$ space is a Hilbert space whose inner product is denoted by $(\cdot, \cdot)$.

Let $\alpha=\left(\alpha_{1}, \alpha_{2}, \ldots, \alpha_{d}\right) \in \mathbb{N}^{d}$ be a multi-index with $|\alpha|=\alpha_{1}+\alpha_{2}+\cdots+\alpha_{d}$, and let $\partial^{\alpha}$ be the differential operator such that

$\partial^{\alpha}=\left(\frac{\partial}{\partial x_{1}}\right)^{\alpha_{1}} \cdots\left(\frac{\partial}{\partial x_{d}}\right)^{\alpha_{d}}$.

For $m \geq 0$ and $1 \leq p \leq \infty$, we define $W^{m, p}(\Omega)$ (see $[1,48])$ to be the Sobolev space of $m$ derivatives in $L^{p}(\Omega)$ whose norm is defined by

$$
\begin{aligned}
& \|f\|_{W^{m, p}(\Omega)}=\left(\sum_{|\alpha| \leq m}\left\|\partial^{\alpha} f\right\|_{L^{p}(\Omega)}^{p}\right)^{1 / p} \text { for } 1 \leq p<\infty, \\
& \|f\|_{W^{m, p}(\Omega)}=\max _{|\alpha| \leq m}\left\|\partial^{\alpha} f\right\|_{L^{\infty}(\Omega)}, \quad \text { for } p=\infty,
\end{aligned}
$$

where $\partial^{\alpha}$ is understood in the distributional sense. In the particular case of $p=2, W^{m, p}(\Omega)=H^{m}(\Omega)$. Let $\mathcal{C}_{c}^{\infty}(\Omega)$ be the space of infinitely differentiable functions with compact support in $\Omega$. Then $W_{0}^{m, p}(\Omega)$ (analogously, $H_{0}^{m}(\Omega)$ when $\left.p=2\right)$ is defined as the closure of $\mathcal{C}_{c}^{\infty}(\Omega)$ in $W^{m, p}(\Omega)$. The dual spaces of $H^{s}(\Omega)$ and $H_{0}^{s}(\Omega)$ will be denoted by $\left(H^{s}(\Omega)\right)^{\prime}$ and $H^{-s}(\Omega)$, respectively. For any space $X$, we shall denote the vector space $X^{d}$ by its bold letter $\boldsymbol{X}$. For example, $\left(L^{2}(\Omega)\right)^{d}$ is denoted by $L^{2}(\Omega),\left(H^{m}(\Omega)\right)^{d}$ by $H^{m}(\Omega)$, etc. Consequently, in order to distinguish scalarvalued fields, such as the pressure $p$, from vector-valued fields, such as the velocity $u$, we denote them by roman letters and bold-face letters, respectively.

For a real Banach space $X, L^{p}(0, T ; X)$ denotes the space of $X$-valued functions $f$ defined on $(0, T)$ such that $\|\cdot\|_{L^{p}(0, T ; X)}=\left(\int_{0}^{T}\|f\|_{X}^{p}\right)^{1 / p}<\infty \cdot C^{1}([0, T] ; X)$ is the

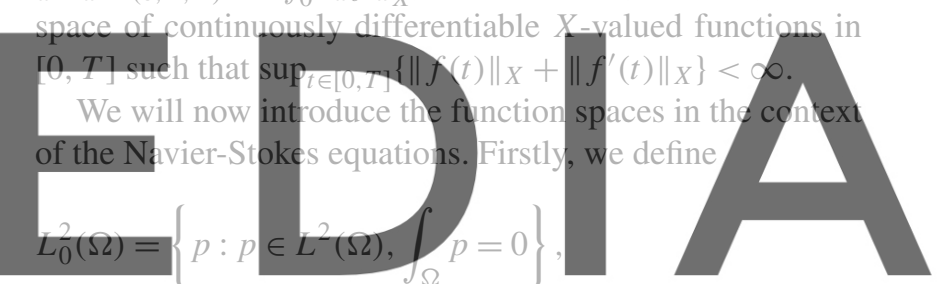

$\vartheta=\left\{v \in C^{\infty}(\Omega) ; \nabla \cdot v=0\right\}$.

download the version without the watermark Then, let $\boldsymbol{H}$ and $\boldsymbol{V}$ be the closure of $\boldsymbol{\vartheta}$ in $\boldsymbol{L}^{2}(\Omega)$ and $\boldsymbol{H}_{0}^{1}(\Omega)$, respectively, characterized by

$$
\begin{aligned}
& \boldsymbol{H}=\left\{\boldsymbol{u} \in \boldsymbol{L}^{2}(\Omega) ; \nabla \cdot \boldsymbol{u}=0, \boldsymbol{u} \cdot \mathbf{n}=0 \text { on } \partial \Omega\right\}, \\
& \boldsymbol{V}=\left\{\boldsymbol{u} \in \boldsymbol{H}^{1}(\Omega) ; \nabla \cdot \boldsymbol{u}=0, \boldsymbol{u}=\mathbf{0} \text { on } \partial \Omega\right\}
\end{aligned}
$$

provided that $\Omega$ is Lipschitz [55].

\subsection{The Ericksen-Leslie Equations}

Let $\Omega$ be a bounded open subset of $\mathbb{R}^{d}(d=2$ or 3 ) with boundary $\partial \Omega$, and $T>0$ the final time of observation. We will use the notation $Q=\Omega \times(0, T), \Sigma=\partial \Omega \times(0, T)$, and $\boldsymbol{n}$ the unit outward normal to $\partial \Omega$. The Ericksen-Leslie equations for the flow of a nematic liquid crystal can be written as

$\begin{cases}\partial_{t} \boldsymbol{u}+(\boldsymbol{u} \cdot \nabla) \boldsymbol{u}-v \Delta \boldsymbol{u} & \\ \quad+\nabla p+\lambda \nabla \cdot\left((\nabla \boldsymbol{d})^{t} \nabla \boldsymbol{d}\right)=\mathbf{0} & \text { in } Q, \\ \nabla \cdot \boldsymbol{u}=0 & \text { in } Q, \\ \partial_{t} \boldsymbol{d}+(\boldsymbol{u} \cdot \nabla) \boldsymbol{d}-\gamma \Delta \boldsymbol{d}-\gamma|\nabla \boldsymbol{d}|^{2} \boldsymbol{d}=\mathbf{0} & \text { in } Q, \\ |\boldsymbol{d}|=1 & \text { in } Q,\end{cases}$ 
where $\boldsymbol{u}: Q \rightarrow \mathbb{R}^{d}$ denotes the solenoidal velocity field, $p: Q \rightarrow \mathbb{R}$ denotes the pressure, and $\boldsymbol{d}: Q \rightarrow \mathbb{R}^{d}$ represents the director field that describes the average molecular alignment.

The parameter $v>0$ is a constant depending on the fluid viscosity, $\lambda>0$ is an elastic constant and $\gamma>0$ is a relaxation time constant. The expression $(\boldsymbol{u} \cdot \nabla) \boldsymbol{u}$ is the vector function whose $i$ th component is $\sum_{j=1}^{d} \boldsymbol{u}_{j} \cdot \partial_{j} \boldsymbol{u}_{i}, \nabla \boldsymbol{d}$ is the gradient operator $\left(\partial_{j} \boldsymbol{d}_{i}\right),(\nabla \boldsymbol{d})^{t}$ denotes its transpose and $|\boldsymbol{d}|=|\boldsymbol{d}(\boldsymbol{x}, t)|$ is the Euclidean norm in $\mathbb{R}^{d}$.

From the point of view of continuum mechanics, system (3) is essentially the simplest set of equations describing the motion of a nematic liquid crystal. System (3) was proposed by Lin in [37] from the macroscopic hydrodynamic theory of nematic liquid crystals established by Ericksen [18, 19] and Leslie $[35,36]$. Since the original Ericksen-Leslie equations are mathematically untractable, further simplifications must be done in order to reduce the many reactive coupling terms between the fields $\boldsymbol{d}$ and $\boldsymbol{u}$ in the Oseen-Frank free energy functional. Although one could argue that system (3) is over-simplified, it keeps the core of the mathematical structure, such as strong nonlinearities and constraints, as well as the physical structure, such as the anisotropic effect of elasticity on the velocity vector field

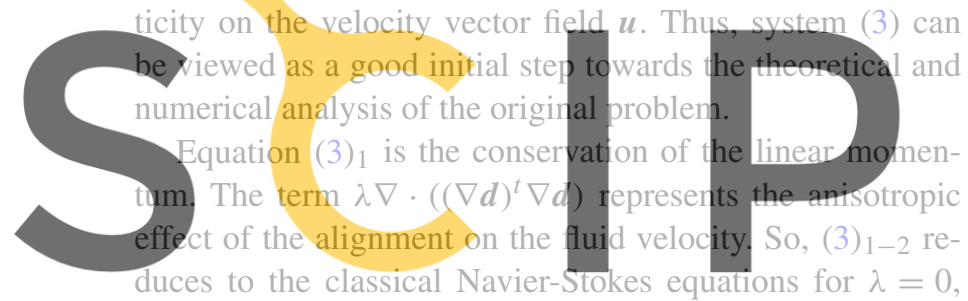

which indicates that the molecular centers of mass have no

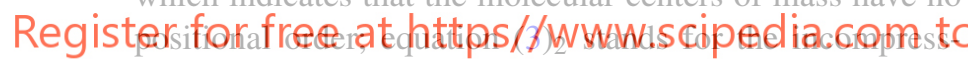
ibility of the fluid. Equation (3) 3 is the conservation of the angular momentum. Equation (3) 4 indicates that $d$ is not a state variable, it only describes the orientation of the nematic liquid crystal molecules.

As explained above, liquid crystals are in general sensitive to temperature. Herein, we only consider the thermally uncoupled model (3). To these equations we will add homogeneous Dirichlet and Neumann boundary conditions for the velocity and director vector fields, respectively:

$\boldsymbol{u}(\boldsymbol{x}, t)=0, \quad \partial_{n} \boldsymbol{d}(\boldsymbol{x}, t)=0 \quad$ on $(\boldsymbol{x}, t) \in \Sigma$,

and the initial conditions

$\boldsymbol{u}(\boldsymbol{x}, 0)=\boldsymbol{u}_{0}(\boldsymbol{x}), \quad \boldsymbol{d}(\boldsymbol{x}, 0)=\boldsymbol{d}_{0}(\boldsymbol{x}) \quad$ on $\boldsymbol{x} \in \Omega$.

Here $\boldsymbol{u}_{0}: \Omega \rightarrow \mathbb{R}^{d}$ and $\boldsymbol{d}_{0}: \Omega \rightarrow \mathbb{R}^{d}$ are given functions such that $\nabla \cdot \boldsymbol{u}_{0}=0$ and $\left|\boldsymbol{d}_{0}\right|=1$ in $\Omega$. Pre-twist boundary conditions for the director $\boldsymbol{d}$ modeled by non-homogeneous Dirichlet conditions as for the pixels could be straightforwardly considered but we have not included them here for the sake of clarity.
A natural question that arises is: Does system (3) have an energy estimate? In other words, we want to know if system (3) is energetically dissipative. The next analysis is just done formally. Let us start observing that the elastic stress tensor can be re-written [38] as

$\lambda \nabla \cdot\left((\nabla \boldsymbol{d})^{t} \nabla \boldsymbol{d}\right)=\lambda \nabla\left(\frac{1}{2}|\nabla \boldsymbol{d}|^{2}\right)-\lambda(\nabla \boldsymbol{d})^{t}(-\Delta \boldsymbol{d})$,

where $\lambda / 2|\nabla \boldsymbol{d}|^{2}$ can be incorporated as part of the pressure. Then, multiply (3) ${ }_{1}$ by $\boldsymbol{u}$ and (3) ${ }_{3}$ by $\lambda\left(-\Delta \boldsymbol{d}-|\nabla \boldsymbol{d}|^{2} \boldsymbol{d}\right)$, and integrate over $\Omega$. Provided that $|\boldsymbol{d}|=1$ can be proved previously in some way, it follows that

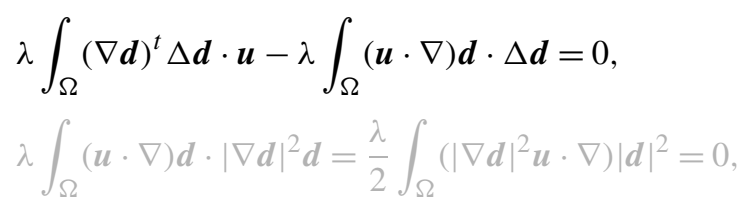

and
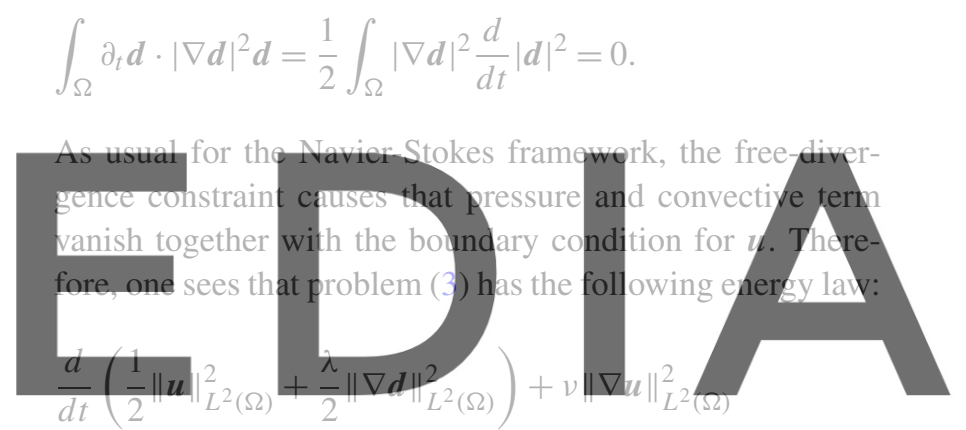

\section{dowritobad the dierision without the wateriphark}

Here we have used the homogeneous Dirichlet and Neumann boundary condition for the velocity and vector director field, respectively, to eliminate the boundary terms stemmed from integration by parts.

Clearly, the sphere constraint over $\boldsymbol{d}$ has been crucial to obtain the energy equality (7). This energy equality, which has been formally established, is called the first energy equality for (3). It expresses the balance of energy in the system between the kinetic and elastic energies, i.e. the dependence between the linear and angular momentum equations along with the incompressibility and unit $\mathrm{Eu}-$ clidean norm constraint. Since any body force is considered, the rate of decay of the kinetic and elastic energy is dictated by the viscous term $v\|\nabla \boldsymbol{u}\|_{L^{2}(\Omega)}^{2}$ and by the term $\lambda \gamma\left\|\Delta \boldsymbol{d}+|\nabla \boldsymbol{d}|^{2} \boldsymbol{d}\right\|_{L^{2}(\Omega)}^{2}$ which is the $L^{2}(\Omega)$-norm of the residual with respect to the steady equation (2).

What one may now expect is that the constraint $|\boldsymbol{d}|=1$ would be a consequence of (3) $)_{1-3}$, such as the maximum principle for convection-diffusion equations, since it is an algebraic constraint. But this is not the case. First of all, we 
establish an equation for $|\boldsymbol{d}|$. If we take the dot product of (3) 3 with $\boldsymbol{d}$, this leads to

$$
\begin{aligned}
& \frac{1}{2} \frac{d}{d t}|\boldsymbol{d}(t)|^{2}+\frac{1}{2}(\boldsymbol{u}(t) \cdot \nabla)|\boldsymbol{d}(t)|^{2} \\
& \quad-\gamma \Delta \boldsymbol{d}(t) \cdot \boldsymbol{d}(t)-\gamma|\nabla \boldsymbol{d}(t)|^{2}|\boldsymbol{d}(t)|^{2}=0 .
\end{aligned}
$$

Next, observe that

$\frac{1}{2} \Delta|\boldsymbol{d}|^{2}=\Delta \boldsymbol{d} \cdot \boldsymbol{d}+|\nabla \boldsymbol{d}|^{2}$.

Then,

$$
\begin{aligned}
\frac{1}{2} & \frac{d}{d t}|\boldsymbol{d}(t)|^{2}+\frac{1}{2}(\boldsymbol{u}(t) \cdot \nabla)|\boldsymbol{d}(t)|^{2} \\
& \quad-\frac{\gamma}{2} \Delta|\boldsymbol{d}(t)|^{2}+\gamma|\nabla \boldsymbol{d}(t)|^{2}\left(1-|\boldsymbol{d}(t)|^{2}\right)=0 .
\end{aligned}
$$

$$
\begin{aligned}
& \text { Equivalently, we have } \\
& \begin{array}{l}
\frac{1}{2} \frac{d}{d t}\left(|d(t)|^{2}-1\right)+\frac{1}{2}(u(t) \cdot \nabla)\left(|d(t)|^{2}-1\right) \\
\quad-\frac{\gamma}{2} \Delta\left(|d(t)|^{2}-1\right)-\gamma|\nabla d(t)|^{2}\left(|d(t)|^{2}-1\right)=0 .
\end{array}
\end{aligned}
$$

Finally, if we multiply this equality by $|d|^{2}-1$ and integrate

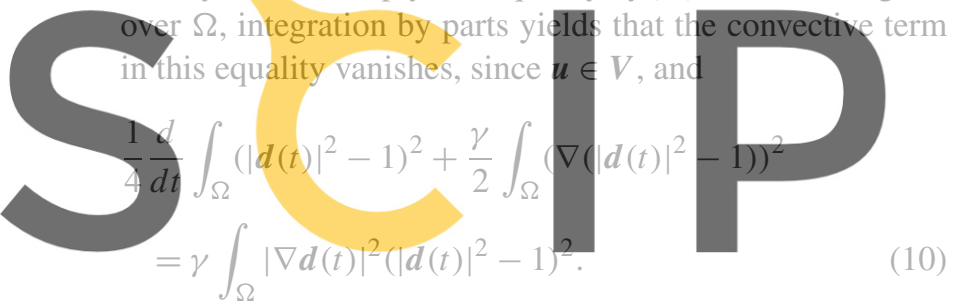

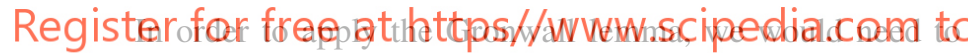
assume a regularity over $d$ stronger than the one obtained from the energy estimate (7). To be more precise, $\nabla d \in$ $L^{2}\left(0, T ; \boldsymbol{L}^{\infty}(\Omega)\right)$. Subsequently, we are not able to prove the maximum principle this way; and hence the sphere constraint must be imposed in system (3).

The energy law (7) shows the proper functional spaces where a feasible definition of global-in-time weak solutions for (3) might be defined.

Definition 1 A pair $(\boldsymbol{u}, \boldsymbol{d})$ is said to be a weak solution of (3)-(4)-(5) in $(0, T)$ if:

(a)

$$
\begin{aligned}
& \boldsymbol{u} \in L^{\infty}(0, T ; \boldsymbol{H}) \cap L^{2}(0, T ; \boldsymbol{V}), \\
& \boldsymbol{d} \in L^{\infty}\left(0, T ; \boldsymbol{H}^{1}(\Omega)\right), \\
& |\boldsymbol{d}(\boldsymbol{x}, t)|=1, \text { a.e. }(\boldsymbol{x}, t) \in Q .
\end{aligned}
$$

(b) $\forall \boldsymbol{\phi} \in C^{1}\left([0, T] ; \boldsymbol{V} \cap \boldsymbol{W}^{1, \infty}(\Omega)\right)$ such that $\boldsymbol{\phi}(T)=0$,

$$
\int_{0}^{T}\left\{-\left(\boldsymbol{u}, \partial_{t} \boldsymbol{\phi}\right)+((\boldsymbol{u} \cdot \nabla) \boldsymbol{u}, \boldsymbol{\phi})+v(\nabla \boldsymbol{u}, \nabla \boldsymbol{\phi})\right.
$$

$$
\left.-\lambda\left((\nabla \boldsymbol{d})^{t} \nabla \boldsymbol{d}, \nabla \boldsymbol{\phi}\right)\right\} d t=\left(\boldsymbol{u}_{0}, \boldsymbol{\phi}(0)\right) .
$$

(c) $\forall \psi \in C^{1}\left([0, T] ; \boldsymbol{H}_{0}^{1}(\Omega) \cap \boldsymbol{L}^{\infty}(\Omega)\right)$ such that $\boldsymbol{\psi}(T)=0$,

$$
\begin{gathered}
\int_{0}^{T}\left\{-\left(\boldsymbol{d}, \partial_{t} \boldsymbol{\psi}\right)+((\boldsymbol{u} \cdot \nabla) \boldsymbol{d}, \boldsymbol{\psi})+\gamma(\nabla \boldsymbol{d}, \nabla \boldsymbol{\psi})\right. \\
\left.-\gamma\left(|\nabla \boldsymbol{d}|^{2} \boldsymbol{d}, \boldsymbol{\psi}\right)\right\} d t=\left(\boldsymbol{d}_{0}, \boldsymbol{\psi}(0)\right) .
\end{gathered}
$$

To our knowledge, the existence of a global-in-time weak solution for (3) still remains as an open problem.

System (3) in its present formulation is overdetermined. There are four equations for three unknowns, and none of them seems to be dependent on the rest. Hence, the obtention of an energy estimates from (3) seems to be unaffordable. Of course, this is an important aspect when designing a numerical approximation for (3), because it could affect the robustness and uniqueness of the numerical method.

As observed, (3) (along with (3) 2 ) and (3) 3 (along with (3) 4 ) share a similar structure. But it is worth mentioning that $(3)_{2}$ is a linear differential constraint whereas $(3)_{4}$ is a nonlinear algebraic constraint, which does not imply that the techniques in order to prove some results for one can be useful for the other. Of course, one expects that the constraints

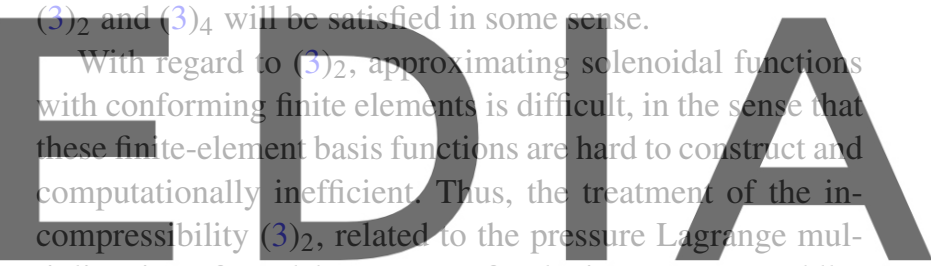

tiplier, is enforced by means of velocity-pressure saddlepoint methods which provide dişcrete divergence-free apdownlard the versignwitheut the watermark

responding velocity-pressure spaces must satisfy a discrete inf-sup condition, in order to get a well-posed discrete problem. This compatibility conditions between spaces can be circumvented via stabilized finite element methods (see e.g. [4]). Alternatively, the incompressibility condition can be penalized and the pressure unknown eliminated. This last approach does not require the computation of the pressure, but consistency is lost and the condition number blows up with the penalty, making the solution of the system too expensive for acceptable values of the penalty parameter (see e.g. [11]).

To enforce the unit Euclidean norm for the director $\boldsymbol{d}$, we can also consider a penalty or a saddle-point approach.

\section{A Penalty Method}

A well-known penalty formulation for (3) uses the GinzburgLandau function $\boldsymbol{f}_{\varepsilon}(\boldsymbol{d})=\varepsilon^{-2}\left(|\boldsymbol{d}|^{2}-1\right) \boldsymbol{d}$, associated with the penalty parameter $\varepsilon>0$. Then, the penalty formulation is obtained from (3) by weakening the constraint $|\boldsymbol{d}|=1$ by $|\boldsymbol{d}| \leq 1$, and replacing the strongly nonlinear term $|\nabla \boldsymbol{d}|^{2} \boldsymbol{d}$ 
by $\boldsymbol{f}_{\varepsilon}(\boldsymbol{d})$. Note that $\boldsymbol{f}_{\varepsilon}$ is the gradient of the scalar potential function

$F_{\varepsilon}(\boldsymbol{d})=\frac{1}{4 \varepsilon^{2}}\left(|\boldsymbol{d}|^{2}-1\right)^{2}$,

that is, $\boldsymbol{f}_{\varepsilon}(\boldsymbol{d})=\nabla_{\boldsymbol{d}} F_{\varepsilon}(\boldsymbol{d})$ for all $\boldsymbol{d} \in \mathbb{R}^{d}$. This fact is basic to obtain an energy estimate for the penalized problem. Thus, we arrive at

$$
\begin{cases}\partial_{t} \boldsymbol{u}+(\boldsymbol{u} \cdot \nabla) \boldsymbol{u}-v \Delta \boldsymbol{u} & \\ \quad+\nabla p+\lambda \nabla \cdot\left((\nabla \boldsymbol{d})^{t} \nabla \boldsymbol{d}\right)=\mathbf{0} & \text { in } Q \\ \nabla \cdot \boldsymbol{u}=0 & \text { in } Q \\ \partial_{t} \boldsymbol{d}+(\boldsymbol{u} \cdot \nabla) \boldsymbol{d}+\gamma\left(\boldsymbol{f}_{\varepsilon}(\boldsymbol{d})-\Delta \boldsymbol{d}\right)=\mathbf{0} & \text { in } Q \\ |\boldsymbol{d}| \leq 1 & \text { in } Q\end{cases}
$$

The energy estimate for (11) was established in [38]. First of all, we have to re-write the elastic stress tensor as in (6) below. Then, (11) 1 is multiplied by $u$ and (11) 2 by $\lambda\left(-\Delta d+f_{\varepsilon}(d)\right)$. After integrating over $\Omega$ and using the fact that

$\lambda \int_{\Omega}(\nabla \boldsymbol{d})^{t} \Delta d \cdot u-\lambda \int_{\Omega}(u \cdot \nabla) d \cdot \Delta d=0$,

$\lambda \int(u \cdot \nabla) d \cdot f_{\varepsilon}(d)=-\lambda \int_{\Omega}(\nabla \cdot u) F_{\varepsilon}(d)=0$,

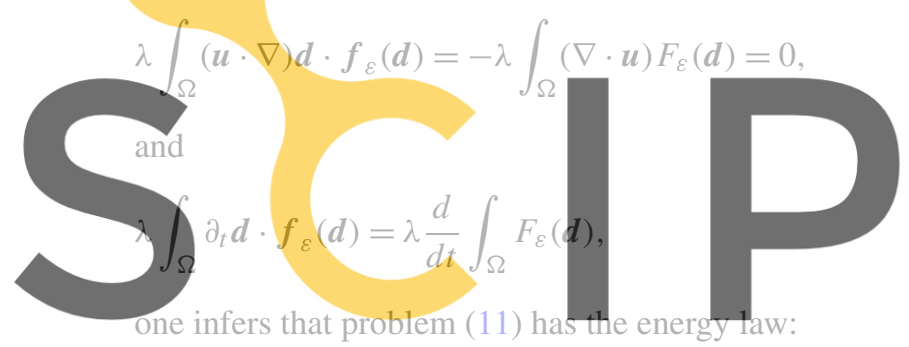

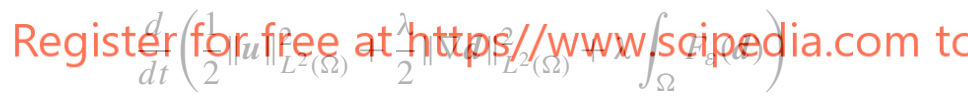

$$
+\nu\|\nabla u\|_{L^{2}(\Omega)}^{2}+\lambda \gamma\left\|-\Delta d+f_{\varepsilon}(d)\right\|_{L^{2}(\Omega)}^{2}=0 .
$$

As far as we know, this is the best energy estimate for (11) independent of $\varepsilon$.

In contrast to what happens for (3), the relaxed constraint $|\boldsymbol{d}| \leq 1$ can now be accomplished $[23,44,50]$ as a maximum principle for convection-diffusion-reaction equations, allowing us to eliminate it from (11). That is, if $\left|\boldsymbol{d}_{0}\right| \leq 1$ in $\Omega$, then $|\boldsymbol{d}(t)| \leq 1$ in $\Omega$ for $t \in(0, T)$. Indeed, if we multiply $(11)_{3}$ by $\boldsymbol{d}$, we see that $|\boldsymbol{d}|$ satisfies

$$
\begin{aligned}
& \frac{1}{2} \frac{d}{d t}|\boldsymbol{d}(t)|^{2}+\frac{1}{2}(\boldsymbol{u}(t) \cdot \nabla)|\boldsymbol{d}(t)|^{2} \\
& \quad-\gamma \Delta \boldsymbol{d}(t) \cdot \boldsymbol{d}(t)+\gamma \boldsymbol{f}_{\varepsilon}(\boldsymbol{d}) \cdot \boldsymbol{d}=0 .
\end{aligned}
$$

The identity (8) leads to

$$
\begin{gathered}
\frac{1}{2} \frac{d}{d t}\left(|\boldsymbol{d}(t)|^{2}-1\right)+\frac{1}{2}(\boldsymbol{u}(t) \cdot \nabla)\left(|\boldsymbol{d}(t)|^{2}-1\right) \\
-\frac{1}{2} \gamma \Delta\left(|\boldsymbol{d}(t)|^{2}-1\right)+\gamma \boldsymbol{f}_{\varepsilon}(\boldsymbol{d}) \cdot \boldsymbol{d} \leq 0,
\end{gathered}
$$

where we have used the fact that $\gamma|\nabla \boldsymbol{d}|^{2} \geq 0$. Testing the above equation by $\left(|\boldsymbol{d}|^{2}-1\right)_{+} \in H^{1}(\Omega)$, with $(\cdot)_{+}$being the positive part, one observes that

$\boldsymbol{f}_{\varepsilon}(\boldsymbol{d}) \cdot \boldsymbol{d}\left(|\boldsymbol{d}|^{2}-1\right)_{+} \geq 0$

and

$$
\begin{aligned}
& \int_{\Omega}\left((\boldsymbol{u} \cdot \nabla)\left(|\boldsymbol{d}|^{2}-1\right)\right)\left(|\boldsymbol{d}|^{2}-1\right)_{+} \\
& \quad=-\frac{1}{2} \int_{\Omega} \nabla \cdot \boldsymbol{u}\left[\left(|\boldsymbol{d}|^{2}-1\right)_{+}\right]^{2}=0
\end{aligned}
$$

and hence

$$
\frac{d}{d t}\left\|\left(|\boldsymbol{d}|^{2}-1\right)_{+}\right\|_{L^{2}(\Omega)}^{2}+\gamma\left\|\nabla\left(|\boldsymbol{d}|^{2}-1\right)_{+}\right\|_{L^{2}(\Omega)}^{2} \leq 0 .
$$

\section{Since $\left(\left|d_{0}\right|^{2}-1\right)_{+}=0$ in $\Omega$, then $|d| \leq 1$ in $Q$.}

Now, the question that arises is how one recovers a solution of system (3) from system (11) as $\varepsilon \rightarrow 0$, at least formally. We follow the ideas in [38] based on those developed for harmonic maps [10, 53]. To do so, we note that equations (3) $1-2$ and (11) $1-2$ are exactly the same. So, the difference between the two approaches strives in the $d$ -

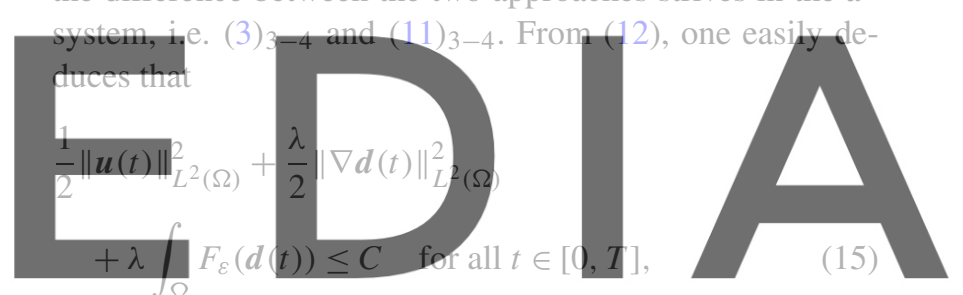

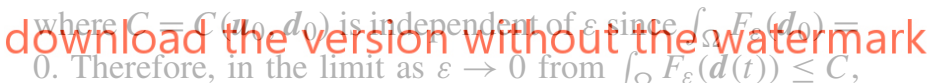
we find $|d|=1$. On the other hand, applying the cross product of (11) with $\boldsymbol{d}$, and passing to the limit as $\varepsilon \rightarrow 0$, we would obtain

$\partial_{t} \boldsymbol{d} \times \boldsymbol{d}+(\boldsymbol{u} \cdot \nabla) \boldsymbol{d} \times \boldsymbol{d}-\gamma \Delta \boldsymbol{d} \times \boldsymbol{d}=0$,

which indicates that $\partial_{t} \boldsymbol{d}+(\boldsymbol{u} \cdot \nabla) \boldsymbol{d}-\gamma \Delta \boldsymbol{d}=0$ is parallel to $\boldsymbol{d}$. Hence, there exists a function $\kappa=\kappa(\boldsymbol{d})$ such that

$\partial_{t} \boldsymbol{d}+(\boldsymbol{u} \cdot \nabla) \boldsymbol{d}-\gamma \Delta \boldsymbol{d}=\kappa(\boldsymbol{d}) \boldsymbol{d}$.

Now, taking the dot product with respect to $\boldsymbol{d}$ and using the fact that $|\boldsymbol{d}|=1$, we find $-\gamma \Delta \boldsymbol{d} \cdot \boldsymbol{d}=\kappa(\boldsymbol{d})$ concluding that $\kappa(\boldsymbol{d})=\gamma|\nabla \boldsymbol{d}|^{2}$, owing to (8).

However, a little bit more can be said about system (11) [8]. Define

$\boldsymbol{w}=-\Delta \boldsymbol{d}+\boldsymbol{f}_{\varepsilon}(\boldsymbol{d})$,

then $\boldsymbol{w} \in L^{2}\left(0, T ; \boldsymbol{L}^{2}(\Omega)\right)$ from (12). Next, multiply (16) by $-\Delta \boldsymbol{d}$ and integrate over $\Omega$ to get 


$$
\begin{aligned}
\|\Delta \boldsymbol{d}\|_{L^{2}(\Omega)}^{2} & =-(\boldsymbol{w}, \Delta \boldsymbol{d})+\left(\boldsymbol{f}_{\varepsilon}(\boldsymbol{d}), \Delta \boldsymbol{d}\right) \\
& \leq\|\boldsymbol{w}\|_{L^{2}(\Omega)}\|\Delta \boldsymbol{d}\|_{L^{2}(\Omega)}-\left(\nabla_{\boldsymbol{d}} \boldsymbol{f}_{\varepsilon}(\boldsymbol{d}) \nabla \boldsymbol{d}, \nabla \boldsymbol{d}\right) \\
& \leq\|\boldsymbol{w}\|_{L^{2}(\Omega)}\|\Delta \boldsymbol{d}\|_{L^{2}(\Omega)}+\frac{C}{\varepsilon^{2}}\|\nabla \boldsymbol{d}\|_{L^{2}(\Omega)}^{2},
\end{aligned}
$$

where in the last line we have used the fact that $|\boldsymbol{d}| \leq 1$ a.e. in $Q$. Young's inequality gives

$$
\|\Delta \boldsymbol{d}\|_{L^{2}(\Omega)}^{2} \leq\|\boldsymbol{w}\|_{L^{2}(\Omega)}^{2}+\frac{C}{\varepsilon^{2}}\|\nabla \boldsymbol{d}\|_{L^{2}(\Omega)}^{2} .
$$

Next, integrating over $(0, T)$ jointly with estimate (15) yield

$\int_{0}^{T}\|\Delta \boldsymbol{d}\|_{L^{2}(\Omega)}^{2} \leq C \varepsilon^{-2}$

This is the best dependence on $\varepsilon$ for the $\boldsymbol{H}^{2}$-norm for the director vector. Therefore, the limiting problem (3) does not hold the $L^{2}\left(0, T ; \boldsymbol{H}^{2}(\Omega)\right)$ regularity as $\varepsilon \rightarrow 0$.

The following estimate can be also proved [44] by multiplying $(11)_{1}$ by $\boldsymbol{u},(11)_{3}$ by $-\lambda \Delta d$, and bounding the term $\lambda \gamma\left(\boldsymbol{f}_{\varepsilon}(\boldsymbol{d}), \boldsymbol{d}\right)$ as before:

\section{$\frac{d}{d t}\left(\frac{1}{2}\|\boldsymbol{u}\|_{L^{2}(\Omega)}^{2}+\frac{\lambda}{2}\|\nabla d\|_{L^{2}(\Omega)}^{2}\right)$}

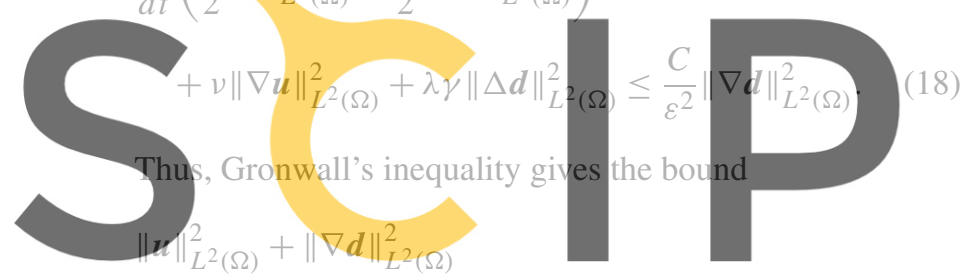

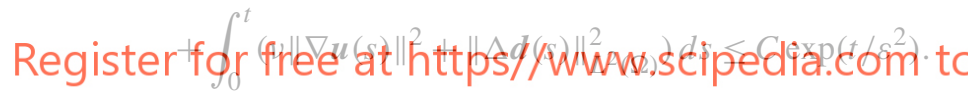

However, the dependence with respect to $\varepsilon$ can be improved by bounding

$\gamma \lambda\left(\boldsymbol{f}_{\varepsilon}(\boldsymbol{d}), \Delta \boldsymbol{d}\right) \leq \frac{\gamma \lambda}{2}\|\Delta \boldsymbol{d}\|_{L^{2}(\Omega)}^{2}+\frac{C}{\varepsilon^{4}}$,

where we have used the fact that $|\boldsymbol{d}| \leq 1$ in $\Omega$. Thus, we have

$$
\begin{aligned}
& \frac{d}{d t}\left(\frac{1}{2}\|\boldsymbol{u}\|_{L^{2}(\Omega)}^{2}+\frac{\lambda}{2}\|\nabla \boldsymbol{d}\|_{L^{2}(\Omega)}^{2}\right) \\
& \quad+v\|\nabla \boldsymbol{u}\|_{L^{2}(\Omega)}^{2}+\lambda \gamma\|\Delta \boldsymbol{d}\|_{L^{2}(\Omega)}^{2} \leq \frac{C}{\varepsilon^{4}} .
\end{aligned}
$$

The energy estimate (18) is better in order to obtain error estimates, but the energy estimate (19) is more adequate from the point of view of stability.

The energy estimate (12) jointly with (17) allows then to define global-in-time weak solutions in the following functional frame.

Definition 2 A pair $(\boldsymbol{d}, \boldsymbol{u})$ is called a weak solution of (11)(4)-(5) in $(0, T)$ if: (a)

$$
\begin{aligned}
& \boldsymbol{u} \in L^{\infty}(0, T ; \boldsymbol{H}) \cap L^{2}(0, T ; \boldsymbol{V}), \\
& \boldsymbol{d} \in L^{\infty}\left(0, T ; \boldsymbol{H}^{1}(\Omega)\right) \cap L^{2}\left(0, T ; \boldsymbol{H}^{2}(\Omega)\right), \\
& |\boldsymbol{d}(\boldsymbol{x}, t)| \leq 1, \quad \text { a.e. }(\boldsymbol{x}, t) \in Q .
\end{aligned}
$$

(b) $\forall \boldsymbol{\phi} \in C^{1}([0, T] ; \boldsymbol{V})$ such that $\boldsymbol{\phi}(T)=0$,

$$
\begin{gathered}
\int_{0}^{T}\left\{-\left(\boldsymbol{u}, \partial_{t} \boldsymbol{\phi}\right)+((\boldsymbol{u} \cdot \nabla) \boldsymbol{u}, \boldsymbol{\phi})+v(\nabla \boldsymbol{u}, \nabla \boldsymbol{\phi})\right. \\
\left.-\lambda\left((\nabla \boldsymbol{d})^{t} \nabla \boldsymbol{d}, \nabla \boldsymbol{\phi}\right)\right\} d t=\left(\boldsymbol{u}_{0}, \boldsymbol{\phi}(0)\right)
\end{gathered}
$$

(c)

$$
\begin{aligned}
& \partial_{t} \boldsymbol{d}+\boldsymbol{u} \cdot \nabla \boldsymbol{d}-\gamma \Delta \boldsymbol{d}+\gamma \boldsymbol{f}_{\varepsilon}(\boldsymbol{d})=\mathbf{0} \quad \text { a.e. in } Q, \\
& \boldsymbol{d}(0)=\boldsymbol{d}_{0} \quad \text { a.e. in } \Omega .
\end{aligned}
$$

So far, the best convergence from (11) to (3), based on an energy method and a compactness result, is towards measure-valued solutions. By a semi-Galerkin approach, the approximate solutions are constructed so that the energy law (12) holds and hence stability is attained in $L^{\infty}\left(0, T ; \boldsymbol{H}^{1}(\Omega)\right)$ for the director field and in $L^{2}(0, T ; \boldsymbol{V}) \cap$ $L^{\infty}(0, T ; \boldsymbol{H})$ for the velocity field independent of $\varepsilon$. It is

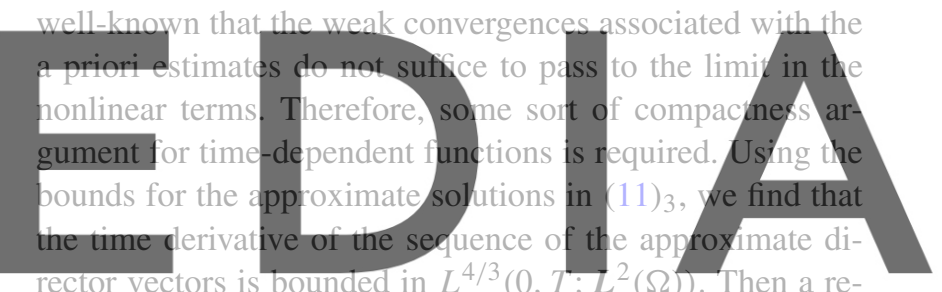

sult of compactness $[42,43]$ shows that the sequence of

down mad the versiondwithout the waternnark

$L^{q}\left(0, T ; L^{r}(\Omega)\right)$ with $1 \leq r<6$ and $1 \leq q<\infty$. Unfortunately, this compactness is too weak to pass to the limit in the elastic tensor $(\nabla \boldsymbol{d})^{t} \nabla \boldsymbol{d}$, since it is only bounded in $L^{\infty}\left(0, T ; L^{1}(\Omega)\right)$. Therefore, the elastic tensor only tends to a certain measure (see $[38,39,47])$. One way to identify the tensor in the limit in $L^{\infty}\left(0, T ; L^{1}(\Omega)\right)$ would involve a $\boldsymbol{H}^{2}(\Omega)$ regularity independent of $\varepsilon$. However, the best bound in this sense depends polynomially on $\varepsilon$ (see (17)).

\section{A Lagrange Multiplier Method}

This sort of method introduces a new variable, the Lagrange multiplier $q$ that allows to enforce the sphere condition $|\boldsymbol{d}|=1$. The saddle-point formulation of problem (3) reads as follows:

$\begin{cases}\partial_{t} \boldsymbol{u}+(\boldsymbol{u} \cdot \nabla) \boldsymbol{u}-v \Delta \boldsymbol{u} & \\ \quad+\nabla p+\lambda \nabla \cdot\left((\nabla \boldsymbol{d})^{t} \nabla \boldsymbol{d}\right)=\mathbf{0} & \text { in } Q, \\ \nabla \cdot \boldsymbol{u}=0 & \text { in } Q, \\ \partial_{t} \boldsymbol{d}+(\boldsymbol{u} \cdot \nabla) \boldsymbol{d}+\gamma(q \boldsymbol{d}-\Delta \boldsymbol{d})=\mathbf{0} & \text { in } Q, \\ |\boldsymbol{d}|=1 & \text { in } Q .\end{cases}$ 
The energy estimate associated with problem (20) was derived in [5]. Assuming that $|\boldsymbol{d}|=1$ holds, the elastic stress tensor can be written as

$$
\begin{aligned}
\lambda \nabla & \cdot\left((\nabla \boldsymbol{d})^{t} \nabla \boldsymbol{d}\right) \\
\quad & =\frac{\lambda}{2} \nabla\left(|\nabla \boldsymbol{d}|^{2}\right)-\lambda(\nabla \boldsymbol{d})^{t}(-\Delta \boldsymbol{d}+q \boldsymbol{d}),
\end{aligned}
$$

since $(\nabla \boldsymbol{d})^{t}(q \boldsymbol{d})=\frac{1}{2} q \nabla\left(|\boldsymbol{d}|^{2}\right)=0$. If we multiply (20) $)_{1}$ by $\boldsymbol{u}$ and $(20)_{3}$ by $\lambda(-\Delta \boldsymbol{d}+q \boldsymbol{d})$, and integrate over $\Omega$, we have

$$
\begin{aligned}
\frac{d}{d t}( & \left.\frac{1}{2}\|\boldsymbol{u}\|_{L^{2}(\Omega)}^{2}+\frac{\lambda}{2}\|\nabla \boldsymbol{d}\|_{L^{2}(\Omega)}^{2}\right) \\
& +v\|\nabla \boldsymbol{u}\|_{L^{2}(\Omega)}^{2}+\lambda \gamma\|-\Delta \boldsymbol{d}+q \boldsymbol{d}\|_{L^{2}(\Omega)}^{2} \\
= & -\int_{\Omega} \partial_{t} \boldsymbol{d} \cdot q \boldsymbol{d} .
\end{aligned}
$$

To control the right hand side of (22), we take the time derivative of $|\boldsymbol{d}|^{2}=1$. Thus, it follows that $\partial_{t} \boldsymbol{d} \cdot \boldsymbol{d}=0$, i.e. $\partial_{t} \boldsymbol{d}$ and $\boldsymbol{d}$ are orthogonal. Therefore,

\section{$\frac{d}{d t}\left(\frac{1}{2}\|\boldsymbol{u}\|_{L^{2}(\Omega)}^{2}+\frac{\lambda}{2}\|\nabla d\|_{L^{2}(\Omega)}^{2}\right)$}

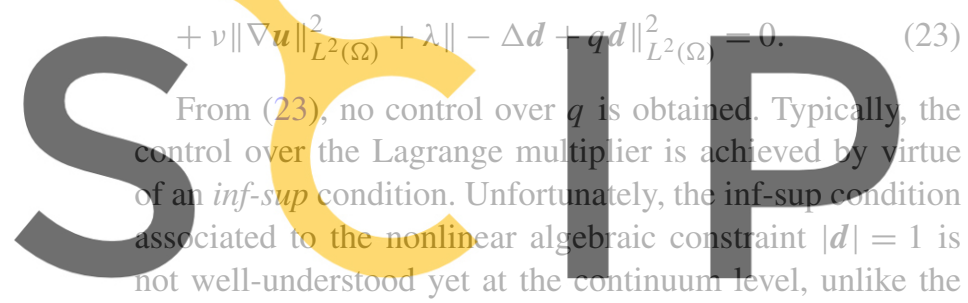
one for the Navier-Stokes equations. The best result is due to

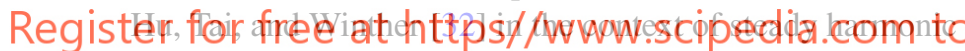
map problems, that is, for $\boldsymbol{u}=0$ and $\partial_{t} \boldsymbol{d}=0$. They proved

$$
\|q\|_{H^{-1}(\Omega)} \leq \alpha \sup _{\overline{\boldsymbol{d}} \in \boldsymbol{H}_{0}^{1}(\Omega) \backslash\{0\}} \frac{\langle q, \boldsymbol{d} \cdot \overline{\boldsymbol{d}}\rangle}{\|\overline{\boldsymbol{d}}\|_{H_{0}^{1}(\Omega)}} \quad \forall q \in H^{-1}(\Omega)
$$

where $\alpha>0$ depends on the $\boldsymbol{W}^{1, \infty}(\Omega)$-norm of $\boldsymbol{d}$. But such a regularity assumption, $\boldsymbol{d} \in \boldsymbol{W}^{1, \infty}(\Omega)$, is not a consequence of (23). The natural inf-sup condition for problem (20) is

$$
\|q\|_{L^{\infty}(\Omega)^{\prime}} \leq \alpha \sup _{\overline{\boldsymbol{d}} \in \boldsymbol{L}^{\infty}(\Omega) \backslash\{0\}} \frac{\langle q, \boldsymbol{d} \cdot \overline{\boldsymbol{d}}\rangle}{\|\overline{\boldsymbol{d}}\|_{L^{\infty}(\Omega)}} \quad \forall q \in L^{\infty}(\Omega)^{\prime}
$$

since $q=-|\nabla \boldsymbol{d}|^{2} \in L^{\infty}\left(0, T ; L^{1}(\Omega)\right)$ and $L^{1}(\Omega) \subset L^{\infty}(\Omega)^{\prime}$ To prove the inf-sup condition (25) we need to assume that $|\boldsymbol{d}|^{2}>1 / \alpha$ a.e. in $\Omega$ for some $\alpha>0$. First of all, we will see that the mapping $\boldsymbol{d} \cdot: \boldsymbol{L}^{\infty}(\Omega) \rightarrow L^{\infty}(\Omega)$ is surjective. Indeed, let $e \in L^{\infty}(\Omega)$, then choose $\overline{\boldsymbol{d}}=\boldsymbol{d} /|\boldsymbol{d}|^{2} e$. Clearly, $e=$ $\boldsymbol{d} \cdot \overline{\boldsymbol{d}} \in L^{\infty}(\Omega)$. Next, observe that $\|\overline{\boldsymbol{d}}\|_{L^{\infty}(\Omega)} \leq \alpha\|e\|_{L^{\infty}(\Omega)}$. Thus, we have

$\|q\|_{L^{\infty}(\Omega)^{\prime}}=\sup _{e \in L^{\infty}(\Omega) \backslash\{0\}} \frac{\langle q, e\rangle}{\|e\|_{L^{\infty}(\Omega)}}$

$$
\leq \alpha \sup _{\overline{\boldsymbol{d}} \in \boldsymbol{L}^{\infty}(\Omega) \backslash\{0\}} \frac{\langle q, \boldsymbol{d} \cdot \overline{\boldsymbol{d}}\rangle}{\|\overline{\boldsymbol{d}}\|_{L^{\infty}(\Omega)}}
$$

for all $q \in L^{\infty}(\Omega)^{\prime}$. But this inf-sup condition is not applicable owing to the presence of $-\boldsymbol{\gamma} \Delta \boldsymbol{d}$ in $(20)_{3}$. Therefore, we need to weaken the norm for the Lagrange multiplier $q$ as follows. Let $q \in\left(\boldsymbol{H}^{1}(\Omega) \cap \boldsymbol{L}^{\infty}(\Omega)\right)^{\prime}$, then one can prove

$\|q\|_{\left(H^{1}(\Omega) \cap L^{\infty}(\Omega)\right)^{\prime}}$

$$
\leq \alpha \sup _{\overline{\boldsymbol{d}} \in \boldsymbol{H}^{1}(\Omega) \cap \boldsymbol{L}^{\infty}(\Omega) \backslash\{0\}} \frac{\langle q, \boldsymbol{d} \cdot \overline{\boldsymbol{d}}\rangle}{\|\nabla \overline{\boldsymbol{d}}\|_{L^{2}(\Omega)}+\|\overline{\boldsymbol{d}}\|_{L^{\infty}(\Omega)}},
$$

where $\boldsymbol{d} \in \boldsymbol{H}^{1}(\Omega) \cap \boldsymbol{L}^{\infty}(\Omega)$ such that $|\boldsymbol{d}|^{2}>1 / \alpha$ a.e. in $\Omega$.

\section{A Ginzburg-Landau-Lagrange Method}

The penalized Ginzburg-Landau problem can also be stated in a saddle-point framework. This way, we can consider both the penalized and limit problem in a unified frame. This method consists in penalizing problem (20) as follows:

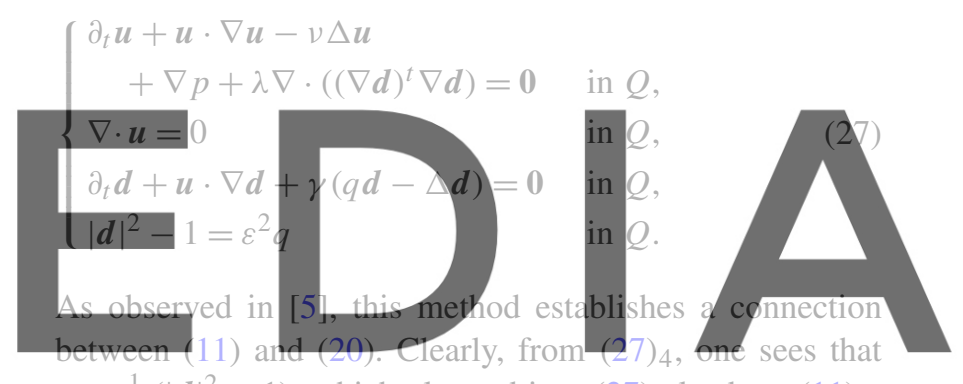

$q=\frac{1}{c^{2}}\left(|d|^{2}-1\right)$, which plugged into (27) 3 leads to (11) 3 .

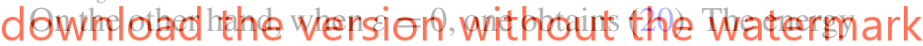
estimate for (27) can be achieved analogously to the energy estimate (23) given by the Lagrange method (20) (see [5]). We have:

$$
\begin{aligned}
& \frac{d}{d t}\left(\frac{1}{2}\|\boldsymbol{u}\|_{L^{2}(\Omega)}^{2}+\frac{\lambda}{2}\|\nabla \boldsymbol{d}\|_{L^{2}(\Omega)}^{2}+\frac{\varepsilon^{2}}{4}\|q\|_{L^{2}(\Omega)}^{2}\right) \\
& \quad+v\|\nabla \boldsymbol{u}\|_{L^{2}(\Omega)}^{2}+\lambda \gamma\|-\Delta \boldsymbol{d}+q \boldsymbol{d}\|_{L^{2}(\Omega)}^{2}=0 .
\end{aligned}
$$

The numerical approximation of the Ericksen-Leslie equations is difficult due to the following reasons:

1. The linear and angular momentum equations are nonlinear.

2. It involves two constraints, the incompressibility condition $(3)_{2}$ and the sphere condition (3) 4 , which is nonconvex.

3. The large number of unknowns that appear in the Ericksen-Leslie equations, due to the coupling between the nonlinear terms and the constraint conditions. So, its numerical approximation and more specifically the solution of the resulting linear systems is computationally expensive. 
Upon analyzing a numerical scheme we must take into account two things:

1. Stability. In numerical analysis, the terminology of stability is frequently associated with bounding the approximate solutions in certain norms, which are related to the energy of the model in question. Such estimates are called a priori or energy estimates. Moreover, the existence and uniqueness of approximate solutions is based on them.

2. Convergence. Two types of convergence results for approximate solutions can be considered. Compactness shows that the approximate solutions converge to a weak solutions under minimum regularity of the data, but no rate of convergence can be guaranteed. On the other hand, if a solution with more regularity than the one provided by the energy estimate is fixed, a priori error estimates can be performed, establishing rates of convergence.

System (3) $1-2$ becomes the classical Navier-Stokes equations plus an elastic stress tensor $\lambda \nabla \cdot\left((\nabla d)^{t} \nabla \boldsymbol{d}\right)$. Therefore, we cannot expect better results than those known for the Navier-Stokes equations (see [26, 55]). Since the

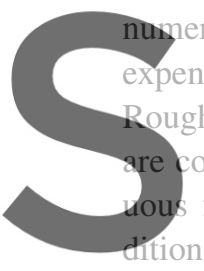
erical approximation of fl nsive, efficient low-order oughly speaking, the discrete functions, satisfying the This sort of method pr
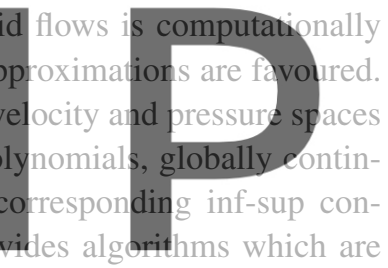

easy to implement, well-conditioned with respect to the dis-

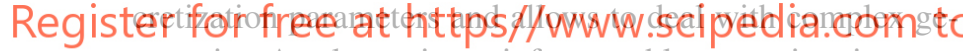
ometries. An alternative to inf-sup stable approximations are the residual-based stabilization techniques (see e.g. [33]), that allow to choose the velocity and pressure spaces without the need to satisfy any compatibility condition and solves the singularly perturbed nature of the problem at hand for convection dominant flows.

With regard to the approximation of (3) $3-4$, each method explained above will give rise to different variational formulations to be approximated by finite-dimensional spaces. In general, we will consider globally continuous piecewise polynomial functions, even though the velocity and director vector regularity are different. A notable exception is the method in [44], where $C^{1}$ approximations are used to approximate the director vector; it was the first numerical scheme to deal with the approximation of (11).

The Ginzburg-Landau method penalizes the constraint (7) ${ }_{4}$ in the $L^{2}$ norm, say $\int_{\Omega}\left(|\boldsymbol{d}|^{2}-1\right)^{2} \leq C \varepsilon^{2}$, from (12). Upon using this penalty method, an important choice is the size of the penalty parameter $\varepsilon$. A very high penalty number leads to ill-conditioned algebraic systems, when the offdiagonal blocks are multiplied by a large number. On the other hand, the rate of convergence is spoiled by $\varepsilon$. Sometimes, the penalization parameter depends on the mesh parameters.

The Lagrange multiplier method introduces a new variable to be computed, which represents an increasement in the dimension of the resulting linear system. In any case, we will see that the numerical approximations of the GinzburgLandau approach require auxiliary variables in order to prove an energy inequality, and in general the dimension of the resulting system is larger than the one of the saddle-point formulation. Furthermore, this method allows one to obtain numerical approximations of the original problem (without penalty) satisfying an energy inequality. On the other hand, the inf-sup condition must be satisfied in order to be wellposed (see [32]). As for the incompressibility condition, the constraint (11) 4 is satisfied in a discrete (weak) sense. Let us remark that, as far as we know, there are no finite element spaces capable to satisfy the restriction pointwise in general. E.g. we can straightforwardly prove that the only functions that belong to linear finite element spaces and satisfy the sphere constraint pointwise are constant functions.

\subsection{Finite Element Approximation}

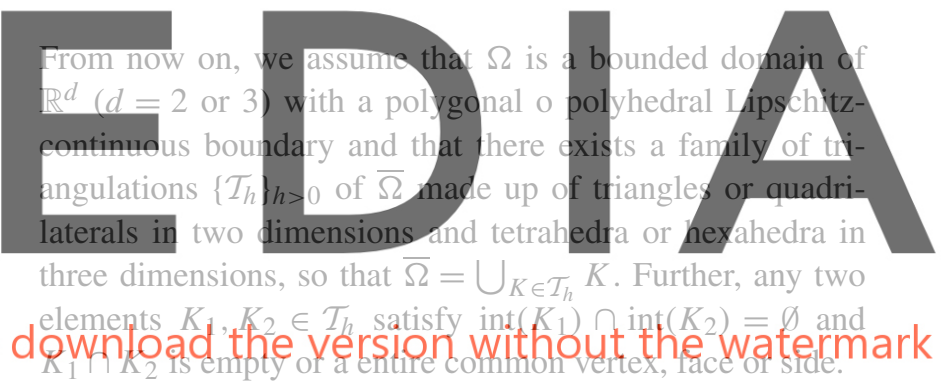

For an arbitrary element $K$, we denote by $h_{K}>0$ the diameter of $K$, with $h=\max _{K \in \mathcal{T}_{h}} h_{K}$, and by $b_{K}$ the radius of the largest ball inscribed in $K$, with $b=\min _{K \in \mathcal{T}_{h}} b_{K}$. The family of triangulations $\mathcal{T}_{h}$ will be assumed to be quasiunform, i.e. there exists $\rho>0$ such that $b \geq \rho h$ for all $K \in \mathcal{T}_{h}$ and for all $h>0$. The space of polynomials of degree less or equal to $k>0$ in a finite element $K$ is denoted by $\mathcal{P}^{k}(K)$. The space of continuous piecewise polynomials is defined as

$\mathcal{P}_{h}^{k}=\left\{v_{h} \in C^{0}(\bar{\Omega})\right.$ such that $\left.\left.v_{h}\right|_{K} \in \mathcal{P}^{k}(K) \forall K \in \mathcal{T}_{h}\right\}$.

We also denote by $\mathcal{D}_{h}^{k}$ the space of piecewise polynomials of order no larger than $k$ without $C^{0}$ continuity. In particular, $\mathcal{D}_{h}^{0}$ is the space of piecewise constant functions. The notation of the finite element spaces to be used for approximating the primary variables are the following. For the velocity and pressure we let $\left(\boldsymbol{V}_{h}, P_{h}\right)$ to be two Lagrange finite element spaces associated with $\mathcal{T}_{h}$. Otherwise stated, 
the velocity finite element space $\boldsymbol{V}_{h}$ that we consider is $\left(\left(\mathcal{P}_{h}^{1}\right)^{d} \oplus\left(\mathcal{B}_{h}\right)^{d}\right) \cap \boldsymbol{H}_{0}^{1}(\Omega)$, where

$\mathcal{B}_{h}=\left\{v_{b}\right.$ such that $\left.\left.v_{b}\right|_{K} \in \mathcal{P}^{d+1}(K),\left.v_{b}\right|_{\partial K}=0, \forall K \in \mathcal{T}_{h}\right\}$

is the space of bubbles in every element (see e.g. [9]). The pressure space $P_{h}$ is $\mathcal{P}_{h}^{1} \cap L_{0}^{2}(\Omega)$. This velocity-pressure finite element pair is known to satisfy the discrete inf-sup condition

$\left\|p_{h}\right\|_{L_{0}^{2}(\Omega)} \leq \beta \sup _{\boldsymbol{v} \in \boldsymbol{V}_{h} \backslash\{0\}} \frac{\left(q_{h}, \nabla \cdot \boldsymbol{v}\right)}{\|\boldsymbol{v}\|_{H^{1}(\Omega)}} \quad \forall p_{h} \in P_{h}$,

for $\beta>0$ uniform with respect to $h$. On the other hand, for the director field, we usually choose $\boldsymbol{D}_{h}$ to be again a Lagrange finite element space, i.e. $\left(\mathcal{P}_{h}^{1}\right)^{d}$. Otherwise stated, these are the typical choices in the subsequent developments. In any case, the following results can be extended to any other finite element spaces, provided the required infsup conditions are satisfied. Throughout this work we will need to introduce some extra discrete spaces which will describe when necessary.

We shall assume that $\Omega$ has the $W^{2, r} \times W^{1, r}$-elliptic regularity property for the Stokes problem. That is to say, for a

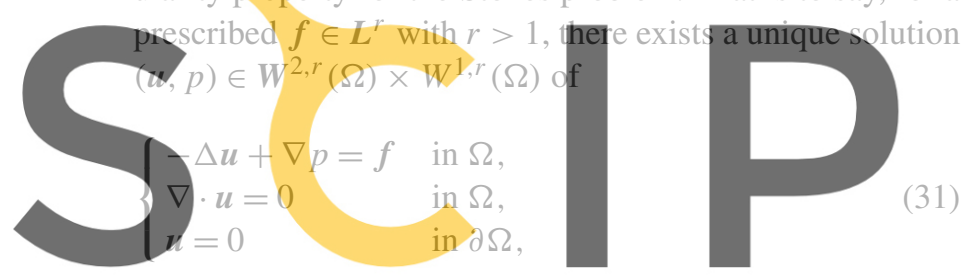

which satisfies the following continuous dependence with Registerpfortof gee at https//www.scipedia.com to

$\|u\|_{W^{2, r}(\Omega)}+\|p\|_{W^{1, r}(\Omega)} \leq C\|f\|_{L^{r}(\Omega)}$.

On the other hand, we also assume that $\Omega$ holds a $W^{2, r_{-}}$ elliptic regularity property for the Neumann problem; given $g \in L^{r}(\Omega)$ with $\int_{\Omega} g=0$, there exists a unique solution $d \in$ $W^{2, r}(\Omega)$ with $\int_{\Omega} d=0$ of

$\begin{cases}-\Delta d=g & \text { in } \Omega \\ \partial_{n} d=0 & \text { in } \partial \Omega\end{cases}$

which satisfies the following continuous dependence with respect to $g$ :

$\|d\|_{W^{2, r}(\Omega)} \leq C\|g\|_{L^{r}(\Omega)}$.

For $r=2$, properties (32) and (34) can be demonstrated for convex $\Omega[13,14,30,34]$ or $C^{1,1}$ boundary [12, 51]. For $r>2$, in two dimensions, properties (32) and (34) hold when $\Omega$ is convex, and $r$ depends on $\partial \Omega[12,30]$. However, in three dimensions, $r$ depends strongly on $\partial \Omega$ so that convexity does not suffice $[12,13]$.
An arbitrary triangulation of an arbitrary domain does not posses the $C^{1,1}$ regularity in general. Therefore, from the point of view of numerical analysis, we are limited to convex domains. Isoparametric elements are needed to generalize to domains with curved boundaries.

Usually, for the Navier-Stokes equation, the above regularity hypotheses are invoked when error estimates are stated, but we will see here that such a regularity is required even for convergence results by compactness.

The initial data are

$\boldsymbol{u}_{0} \in \boldsymbol{H} \quad$ and $\quad \boldsymbol{d}_{0} \in \boldsymbol{H}^{1}(\Omega) \quad$ with $\left|\boldsymbol{d}_{0}\right|=1$ in $\Omega$

when the convergence of the algorithm is established by compactness or

$$
\begin{aligned}
& u_{0} \in H_{0}^{2}(\Omega) \cap V \text { and } \quad d_{0} \in W^{2, r}(\Omega) \\
& \text { with }\left|d_{0}\right|=1 \text { in } \Omega
\end{aligned}
$$

when the convergence of the algorithm is established by error estimates.

All the methods presented herein use a finite difference discretization in time. Let us therefore introduce some nota-
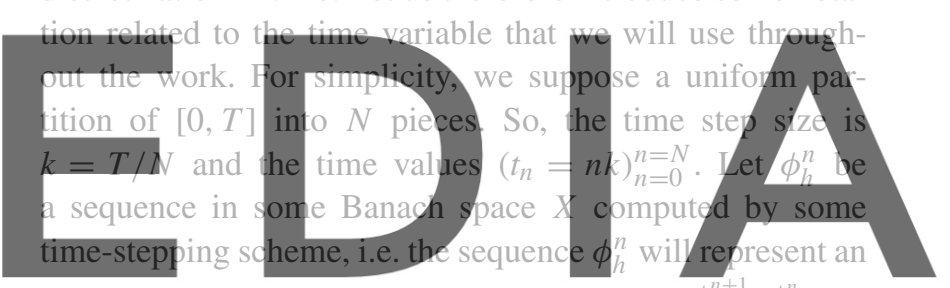

approximation of $\phi\left(t_{n}\right)$. We define $\delta_{t} \phi_{h}^{n+1}=\frac{\phi_{h}^{n+1}-\phi_{h}^{n}}{k}$ and

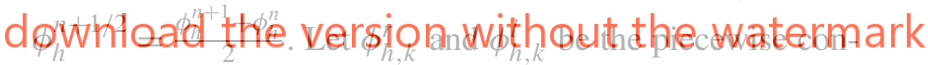

stant interpolation taking the value $\phi_{h}^{n+1}$ or $\phi_{h}^{n}$ on $\left(t_{n}, t_{n+1}\right]$, respectively. Moreover, we define the piecewise linear interpolation $\phi_{h, k} \in C^{0}([0, T] ; X)$ such that $\phi_{h, k}\left(t_{n}\right)=\phi_{h}^{n}$, that is,

$\phi_{h, k}(t)=\frac{t-t_{j}}{k} \phi_{h}^{n+1}+\frac{t_{j+1}-t}{k} \phi_{h}^{n} \quad \forall t \in\left[t_{n}, t_{n+1}\right]$.

Let us introduce some short-hand notation, in order to simplify the writing of the different schemes:

$a(\boldsymbol{u}, \boldsymbol{v})=(\nabla \boldsymbol{u}, \nabla \boldsymbol{v}) \quad$ for all $\boldsymbol{u}, \boldsymbol{v} \in \boldsymbol{H}^{1}(\Omega)$,

$b_{u}(\boldsymbol{u}, q)=(\nabla \cdot \boldsymbol{u}, q) \quad$ for all $\boldsymbol{u} \in \boldsymbol{H}^{1}(\Omega), q \in L^{2}(\Omega)$,

$b_{d}(q, \boldsymbol{d}, \overline{\boldsymbol{d}})=\langle q, \boldsymbol{d} \cdot \overline{\boldsymbol{d}}\rangle$

for all $q \in\left(H^{1}(\Omega)\right)^{\prime}, \boldsymbol{d}, \overline{\boldsymbol{d}} \in \boldsymbol{H}^{1}(\Omega) \cap \boldsymbol{L}^{\infty}(\Omega)$,

$c(\boldsymbol{w}, \boldsymbol{u}, \boldsymbol{v})=\langle(\boldsymbol{w} \cdot \nabla) \boldsymbol{u} \cdot \boldsymbol{v}\rangle \quad$ for all $\boldsymbol{w}, \boldsymbol{u}, \boldsymbol{v} \in \boldsymbol{H}^{1}(\Omega)$.

It is clear that $c(\boldsymbol{w}, \boldsymbol{u}, \boldsymbol{u})=0$ for all $\boldsymbol{w} \in \boldsymbol{V}$. At the discrete level, the approximate velocity does not satisfy the incompressibility condition in a pointwise sense. Thus, in order to keep the skew-symmetry of the trilinear term, the stabilizing 
term $\frac{1}{2}\langle(\nabla \cdot \boldsymbol{u}) \boldsymbol{w}, \boldsymbol{v}\rangle$ is added to the convective term. Then the trilinear form reads:

$\widetilde{c}(\boldsymbol{u}, \boldsymbol{w}, \boldsymbol{v})=\langle(\boldsymbol{u} \cdot \nabla) \boldsymbol{w}, \boldsymbol{v}\rangle+\frac{1}{2}\langle(\nabla \cdot \boldsymbol{u}) \boldsymbol{w}, \boldsymbol{v}\rangle$,

for all $\boldsymbol{w}, \boldsymbol{u}, \boldsymbol{v} \in \boldsymbol{H}^{1}(\Omega)$,

for which $\widetilde{c}(\boldsymbol{w}, \boldsymbol{u}, \boldsymbol{u})=0$ holds for all $\boldsymbol{u}, \boldsymbol{w} \in \boldsymbol{H}_{0}^{1}(\Omega)$.

\section{On the Approximation of the Ginzburg-Landau Problem (11)}

\subsection{Direct Approximation for the Ginzburg-Landau Problem}

\subsubsection{A $\mathrm{H}^{2}$-Conforming Approximation}

The first authors who dealt with the approximation of (11) for two-dimensional domains were Liu and Walkington in [44]. They focused their work in the obtention of error estimates, but they did not avoid the dependence on the penalty parameter $\varepsilon$. The best result for a Ginzburg-Landau-type equation is proved in [22], where the dependence of $\varepsilon$ is of polynomial order. Hence, an interesting open problem is to obtain error estimates independent of the penalty parameter $\varepsilon$, or at least a polynomial dependence.

The starting point of the scheme lies in the following weak reformulation of (11), which is obtained by taking the $L^{2}$ inner product of $\overline{\boldsymbol{u}} \in \boldsymbol{H}_{0}^{1}(\Omega)$ with the linear momentum equation $(11)_{3}$ and the $L^{2}$ inner product of $-\Delta \overline{\boldsymbol{d}}$ (for $\overline{\boldsymbol{d}} \in \boldsymbol{H}^{2}(\Omega)$ ) with the angular momentum equation $(11)_{2}$. After using integration by parts, the problem reads as: find $(\boldsymbol{u}(t), p(t), \boldsymbol{d}(t)) \in \boldsymbol{H}_{0}^{1}(\Omega) \times L_{0}^{2}(\Omega) \times \boldsymbol{H}^{2}(\Omega)$ satisfying

$$
\left\{\begin{array}{c}
\left(\partial_{t} \boldsymbol{u}, \boldsymbol{v}\right)+c(\boldsymbol{u}, \boldsymbol{u}, \boldsymbol{v})+v a(\boldsymbol{u}, \boldsymbol{v}) \\
\quad-b_{u}(p, \overline{\boldsymbol{u}})+\lambda c(\boldsymbol{d}, \boldsymbol{v}, \Delta \boldsymbol{d})=0, \\
b_{u}(\bar{p}, \boldsymbol{u})=0, \\
\left(\partial_{t} \nabla \boldsymbol{d}, \nabla \overline{\boldsymbol{d}}\right)-c(\boldsymbol{u}, \boldsymbol{d}, \Delta \overline{\boldsymbol{d}}) \\
\quad+\gamma\left(\Delta \boldsymbol{d}-\boldsymbol{f}_{\varepsilon}(\boldsymbol{d}), \Delta \overline{\boldsymbol{d}}\right)=0,
\end{array}\right.
$$

for all $(\overline{\boldsymbol{u}}, \bar{p}, \overline{\boldsymbol{d}}) \in \boldsymbol{H}_{0}^{1}(\Omega) \times L_{0}^{2}(\Omega) \times \boldsymbol{H}^{2}(\Omega)$. Observe that the elastic stress tensor has been written as in (21), where the potential term $\frac{\lambda}{2} \nabla\left(|\nabla \boldsymbol{d}|^{2}\right)$ has been absorbed into the definition of the pressure, getting a modified pressure $p \sim$ $p+\frac{\lambda}{2}|\nabla \boldsymbol{d}|^{2}$. In [44], $F_{\varepsilon}$ was truncated to have quadratic growth outside of the unit ball.

In order to obtain a conforming approximation of (35) by a Galerkin finite element approximation, one should use a $\boldsymbol{D}_{h}$ space based on $\boldsymbol{H}^{2}$ conforming finite elements for the third equation in system (35). Thus, a general form for $\boldsymbol{D}_{h}$ is

$\boldsymbol{D}_{h}=\left\{\overline{\boldsymbol{d}}_{h} \in \boldsymbol{C}^{1}(\bar{\Omega}):\left.\overline{\boldsymbol{d}}\right|_{T} \in \mathcal{P}_{k}^{d}\right.$ for all $\left.T \in \mathcal{T}_{h}\right\}$.

In two-dimensional domains, examples of $\boldsymbol{C}^{1}$ finite element spaces are the bicubic Hermite elements or Argyris elements. However, for the first and second equation, one can use a classical pair $\left(V_{h}, P_{h}\right)$ satisfying the inf-sup condition (30), as the one introduced above. In particular, $V_{h}=\mathcal{P}_{h}^{2}$ and $P_{h}=\mathcal{P}_{h}^{1}$ were considered in [44]. With respect to the time integration, e.g. a fully implicit time stepping scheme is used in [44]. All these considerations lead to the following scheme: given $\left(\boldsymbol{u}_{h}^{n}, p_{h}^{n}, \boldsymbol{d}_{h}^{n}\right)$, find $\left(\boldsymbol{u}_{h}^{n+1}, p_{h}^{n+1}, \boldsymbol{d}_{h}^{n+1}\right) \in$ $\boldsymbol{V}_{h} \times P_{h} \times \boldsymbol{D}_{h}$ satisfying

$$
\begin{aligned}
& \left(\delta_{t} \boldsymbol{u}_{h}^{n+1}, \overline{\boldsymbol{u}}_{h}\right)+\widetilde{c}\left(\boldsymbol{u}_{h}^{n}, \boldsymbol{u}_{h}^{n+1}, \overline{\boldsymbol{u}}_{h}\right)+v a\left(\boldsymbol{u}^{n+1}, \overline{\boldsymbol{u}}_{h}\right) \\
& \quad-b_{u}\left(p_{h}^{n+1}, \overline{\boldsymbol{u}}_{h}\right)+\lambda c\left(\overline{\boldsymbol{u}}_{h}, \boldsymbol{d}_{h}^{n+1}, \Delta \boldsymbol{d}_{h}^{n+1}\right)=0, \\
& b_{u}\left(\bar{p}_{h}, \boldsymbol{u}_{h}^{n+1}\right)=0, \\
& \left(\nabla \delta_{t} \boldsymbol{d}_{h}^{n+1}, \nabla \overline{\boldsymbol{d}}_{h}\right)-c\left(\boldsymbol{u}_{h}^{n+1}, \boldsymbol{d}_{h}^{n+1}, \Delta \overline{\boldsymbol{d}}_{h}\right) \\
& \quad+\gamma\left(\Delta \boldsymbol{d}_{h}^{n+1}-\boldsymbol{f}_{\varepsilon}\left(\boldsymbol{d}_{h}^{n+1}\right), \Delta \overline{\boldsymbol{d}}_{h}\right)=0,
\end{aligned}
$$

for all $\left(\overline{\boldsymbol{u}}_{h}, \bar{p}_{h}, \overline{\boldsymbol{d}}_{h}\right) \in \boldsymbol{V}_{h} \times Q_{h} \times \boldsymbol{D}_{h}$. As pointed out in [8], the existence of a discrete solution $\left(\boldsymbol{u}_{h}^{n+1}, p_{h}^{n+1}, \boldsymbol{d}_{h}^{n+1}\right)$ for (36) implies the relation between the time and penalty parameter $k=\mathcal{O}\left(e^{-1 / \varepsilon^{2}}\right)$. This is due to the lack of an energy estimate independent of $\varepsilon$. In [44], the following discrete energy estimate for (36) was derived

$$
\begin{aligned}
& \frac{1}{2}\left\|\boldsymbol{u}_{h}^{n+1}\right\|_{L^{2}(\Omega)}^{2}+\frac{\lambda}{2}\left\|\nabla \boldsymbol{d}_{h}^{n+1}\right\|^{2} \\
& +k \sum_{j=0}^{n}\left(v\left\|\nabla \boldsymbol{u}_{h}^{j+1}\right\|^{2}+\gamma \lambda\left\|\Delta \boldsymbol{d}_{h}^{j+1}\right\|^{2}\right) \\
& \leq C\left(\frac{1}{2}\left\|\boldsymbol{u}_{h}^{0}\right\|^{2}+\frac{\lambda}{2}\left\|\nabla \boldsymbol{d}_{h}^{0}\right\|^{2}\right) \exp \left((n+1) k / \varepsilon^{2}\right)
\end{aligned}
$$

for all $n$.

In order to obtain bounds for $e_{\boldsymbol{u}}^{n}:=\boldsymbol{u}_{h}^{n}-\boldsymbol{u}\left(t_{n}\right)$ and $e_{\boldsymbol{d}}^{n}:=$ $\boldsymbol{d}_{h}^{n}-\boldsymbol{d}\left(t_{n}\right)$, we must assume some sort of regularity for the solution of problem (11):

$\boldsymbol{u} \in C\left(0, T ; \boldsymbol{H}_{0}^{2}(\Omega)\right), \quad \partial_{t} \boldsymbol{u} \in L^{2}\left(0, T ; \boldsymbol{L}^{2}(\Omega)\right)$, $\partial_{t t} \boldsymbol{u} \in L^{2}\left(0, T ; \boldsymbol{H}^{-1}(\Omega)\right), \quad p \in C\left(0, T ; H^{1}(\Omega)\right)$

and

$\boldsymbol{d} \in C\left(0, T ; \boldsymbol{W}^{2,4}(\Omega)\right), \quad \partial_{t} \boldsymbol{d} \in L^{2}\left(0, T ; \boldsymbol{H}^{1}(\Omega)\right)$,

$\partial_{t t} \boldsymbol{d} \in L^{2}\left(0, T ; \boldsymbol{L}^{2}(\Omega)\right)$.

In addition,

$\Delta \boldsymbol{d} \in L^{2}\left(0, T ; \boldsymbol{H}^{1}(\Omega)\right)$. 
In [44] it is shown the following error estimates:

$$
\begin{aligned}
& \left\|\boldsymbol{e}_{\boldsymbol{u}}^{n}\right\|_{L^{2}(\Omega)}^{2}+\left\|e_{\boldsymbol{d}}^{n}\right\|_{H^{1}(\Omega)}^{2} \\
& \quad+k \sum_{m=0}^{n}\left(\left\|e_{\boldsymbol{u}}^{m}\right\|_{H^{1}(\Omega)}^{2}+\left\|e_{\boldsymbol{d}}^{m}\right\|_{H^{2}(\Omega)}^{2}\right) \\
& \leq C\left(k^{2}+h^{2}\right) \quad \text { for all } n,
\end{aligned}
$$

where the constant $C>0$ depends on the penalty parameter $\varepsilon$ and, obviously, on the regularity of the solution. To obtain such error estimates, the differential form of (11) at $t=t_{n+1}$ was used with the corresponding consistency errors. This approach needs the velocity vector $\boldsymbol{u}$ to satisfy an extra compatibility condition established in [31] on the data at $t=0$ to get $\partial_{t t} \boldsymbol{u} \in L^{2}\left(0, T ; \boldsymbol{H}^{-1}(\Omega)\right)$.

Moreover, the regularity for the director vector $\boldsymbol{d}$ requires the Neumann problem (33) holding the regularity (34) for $r=4$, which leads to a restriction on the angles of the boundary $\partial \Omega$.

Clearly, $C^{1}$-finite elements provide high order approximations because they consider a huge degree of approximations. The Argyris element is constructed with polynomials of degree less than or equal to 5 , and it has 21 degree of freedom per triangle for a scalar problem in dimension 2 , whereas the bicubic Hermite element is obtained by products of polynomials of degree less than or equal to 3 , involving 16 degree of freedom for the same case. The huge number of unknowns per element for a vectorial problem in two and three dimensions make this $C^{1}$ approximation extremely intensive in terms of computational cost. Furthermore, these approximations involve the derivatives of the unknowns, complicating their implementation. Although this scheme has demonstrated that can capture the behavior of singularities, it is not appropriate in terms of computation. The use of low order Lagrangian element is favoured for computational efficiency reasons.

\subsubsection{A $\mathrm{H}^{1}$-Conforming Approximation}

The energy estimate (12) can be writen without the need to have $\boldsymbol{d} \in \boldsymbol{H}^{2}(\Omega)$ :

$$
\begin{aligned}
& \frac{d}{d t}\left(\frac{1}{2}\|\boldsymbol{u}\|_{L^{2}(\Omega)}^{2}+\frac{\lambda}{2}\|\nabla \boldsymbol{d}\|_{L^{2}(\Omega)}^{2}+\lambda \int_{\Omega} F_{\varepsilon}(\boldsymbol{d})\right) \\
& \quad+v\|\nabla \boldsymbol{u}\|_{L^{2}(\Omega)}^{2}+\frac{\lambda}{\gamma}\left\|\partial_{t} \boldsymbol{d}+(\boldsymbol{u} \cdot \nabla) \boldsymbol{d}\right\|_{L^{2}(\Omega)}^{2}=0,
\end{aligned}
$$

since $-\Delta \boldsymbol{d}+\boldsymbol{f}_{\varepsilon}(\boldsymbol{d})$, the equation for critical points of the energy $\int_{\Omega} \frac{1}{2}|\nabla \boldsymbol{d}|^{2}+F_{\varepsilon}(\boldsymbol{d})$, has been replaced by the material derivative $\partial_{t} \boldsymbol{d}+(\boldsymbol{u} \cdot \nabla) \boldsymbol{d}$. Lin and Liu presented in [40] one of the simplest time-stepping schemes for the twodimensional Ginzburg-Landau problem (11), in which space is discretized by $H^{1}$-conforming finite elements and time is discretized implicitly with respect to the linear terms and semi-implicitly with respect to the nonlinear terms, except for the anisotropic stress tensor that is fully explicit; so, the penalty term is discretized semi-implicitly. Thus, the resulting scheme reads

$$
\begin{aligned}
& \left(\delta_{t} \boldsymbol{u}_{h}^{n+1}, \overline{\boldsymbol{u}}_{h}\right)+\widetilde{c}\left(\boldsymbol{u}_{h}^{n}, \boldsymbol{u}_{h}^{n+1}, \overline{\boldsymbol{u}}_{h}\right)+v a\left(\boldsymbol{u}^{n+1}, \overline{\boldsymbol{u}}_{h}\right) \\
& \quad-b_{u}\left(p_{h}^{n+1}, \overline{\boldsymbol{u}}_{h}\right)-\lambda\left(\left(\nabla \boldsymbol{d}_{h}^{n}\right)^{t} \nabla \boldsymbol{d}_{h}^{n}, \nabla \overline{\boldsymbol{u}}_{h}\right)=0 \\
& b_{u}\left(\bar{p}_{h}, \boldsymbol{u}_{h}^{n+1}\right)=0 \\
& \left(\delta_{t} \boldsymbol{d}_{h}^{n+1}, \overline{\boldsymbol{d}}_{h}\right)+c\left(\boldsymbol{u}_{h}^{n}, \boldsymbol{d}_{h}^{n+1}, \overline{\boldsymbol{d}}_{h}\right) \\
& \quad+\gamma a\left(\boldsymbol{d}_{h}^{n+1}, \overline{\boldsymbol{d}}_{h}\right)+\frac{\gamma}{\varepsilon^{2}}\left(\left(\left|\boldsymbol{d}_{h}^{n}\right|^{2}-1\right) \boldsymbol{d}_{h}^{n+1}, \overline{\boldsymbol{d}}_{h}\right)=0
\end{aligned}
$$

for all $\left(\overline{\boldsymbol{u}}_{h}, \bar{p}_{h}, \overline{\boldsymbol{d}}_{h}\right) \in \boldsymbol{V}_{h} \times P_{h} \times \boldsymbol{D}_{h}$. In [40], the discrete spaces were $\boldsymbol{V}_{h}=\mathcal{P}_{h}^{2}, P_{h}=\mathcal{P}_{h}^{1}$ and $\boldsymbol{D}_{h}=\mathcal{P}_{h}^{2}$.

Since no energy estimates can be proved independent of the mesh and penalty parameter, the unique solvability of (39) is conditional, that is, there exist a polynomial relation $k=R(\varepsilon, h)$ for which existence and uniqueness of discrete solutions for the scheme (39) may be established.

Obviously, this scheme reduces significantly the computational cost, allowing larger scale numerical simulations. The most important feature is that, at each time step, one only needs to solve a sequence of two decoupled linear problems for the velocity-pressure pair and the director field, separately. However, the authors were not able to derive the discrete analog to the energy estimate (38), which is basic for unconditional stability and also important with regard to the error analysis, if one wants to prove

$$
\begin{aligned}
& \left\|\boldsymbol{e}_{\boldsymbol{u}}^{n}\right\|_{L^{2}(\Omega)}^{2}+\left\|e_{\boldsymbol{d}}^{n}\right\|_{H^{1}(\Omega)}^{2}+k \sum_{m=0}^{n}\left(\left\|e_{\boldsymbol{u}}^{m}\right\|_{H^{1}(\Omega)}^{2}+\left\|e_{\boldsymbol{d}}^{m}\right\|_{H^{2}(\Omega)}^{2}\right) \\
& \quad \leq C\left(k^{2}+h^{2}\right) \quad \text { for all } n .
\end{aligned}
$$

A second algorithm based on the finite element method of characteristics was presented in [40]. Fixed $\left(\boldsymbol{u}_{h}^{n}, p_{h}^{n}, \boldsymbol{d}_{h}^{n}\right)$, find $\left(\boldsymbol{u}_{h}^{n+1}, p_{h}^{n+1}, \boldsymbol{d}_{h}^{n+1}\right) \in \boldsymbol{V}_{h} \times P_{h} \times \boldsymbol{D}_{h}$ such that

$$
\begin{aligned}
& \left(\delta_{t} \boldsymbol{u}_{h}^{n+1}\left(\boldsymbol{X}_{h}^{n}\right), \overline{\boldsymbol{u}}_{h}\right)+v a\left(\boldsymbol{u}^{n+1}, \overline{\boldsymbol{u}}_{h}\right) \\
& \quad-b_{u}\left(p_{h}^{n+1}, \boldsymbol{u}_{h}\right)-\lambda\left(\left(\nabla \boldsymbol{d}_{h}^{n}\right)^{t} \nabla \boldsymbol{d}_{h}^{n}, \nabla \overline{\boldsymbol{u}}_{h}\right)=0, \\
& b_{u}\left(\bar{p}_{h}, \boldsymbol{u}_{h}^{n+1}\right)=0, \\
& \left(\delta_{t} \boldsymbol{d}_{h}^{n+1}\left(\boldsymbol{X}_{h}^{n+1}\right), \overline{\boldsymbol{d}}_{h}\right)+\gamma a\left(\boldsymbol{d}_{h}^{n+1}, \overline{\boldsymbol{d}}_{h}\right) \\
& \quad+\frac{\gamma}{\varepsilon^{2}}\left(\left(\left|\boldsymbol{d}_{h}^{n}\right|^{2}-1\right) \boldsymbol{d}_{h}^{n+1}, \overline{\boldsymbol{d}}_{h}\right)=0,
\end{aligned}
$$

where we have denoted by

$$
\begin{aligned}
& \delta_{t} \boldsymbol{u}_{h}^{n+1}\left(\boldsymbol{X}_{h}^{n}\right)=\frac{\boldsymbol{u}_{h}^{n+1}-\boldsymbol{u}_{h}^{n}\left(\boldsymbol{X}_{h}^{n}\right)}{k} \text { and } \\
& \delta_{t} \boldsymbol{d}_{h}^{n+1}\left(\boldsymbol{X}_{h}^{n+1}\right)=\frac{\boldsymbol{d}_{h}^{n+1}-\boldsymbol{d}_{h}^{n}\left(\boldsymbol{X}_{h}^{n+1}\right)}{k},
\end{aligned}
$$


and $\boldsymbol{X}_{h}^{n}:=\boldsymbol{x}+k \boldsymbol{u}_{h}^{n}(\boldsymbol{x})$ and $\boldsymbol{X}_{h}^{n+1}:=\boldsymbol{x}+k \boldsymbol{u}_{h}^{n+1}(\boldsymbol{x})$, which is a backward Euler time discretization of the characteristic system

$\boldsymbol{X}\left(\boldsymbol{x}, t_{n+1} ; t_{n}\right)=\boldsymbol{x}-\int_{t_{n}}^{t_{n+1}} \boldsymbol{u}\left(\boldsymbol{X}\left(\boldsymbol{x}, t_{n+1} ; t\right), t\right) d t$.

Note that the convective velocity for $\boldsymbol{d}_{h}^{n+1}$ is updated, due to the sequential feature of the method. The discrete spaces $\left(\boldsymbol{V}_{h}, P_{h}, \boldsymbol{D}_{h}\right)$ are considered as above.

The finite element method of characteristics for $(11)_{1-2}$ leads to linear algebraic problems with time-independent matrices, reducing the computational; we can decompose (at least approximately) the associated matrix just once at the beginning of computation. However, the same method does not avoid that $(11)_{3}$ and $(11)_{4}$ lead to time-dependent matrices. For this reason, they proposed an iterative method for (40) in such a way the matrices do not change at each iteration. Given $\boldsymbol{d}_{h}^{n+1, j}$, find $\boldsymbol{d}_{h}^{n+1, j} \in \boldsymbol{D}_{h}$ such that

$$
\begin{gathered}
\left(\frac{\boldsymbol{d}_{h}^{n+1, j+1}-\boldsymbol{d}_{h}^{n}\left(\boldsymbol{X}_{h}^{n+1}\right)}{k}, \overline{\boldsymbol{d}}_{h}\right)+a\left(\boldsymbol{d}_{h}^{n+1, j+1}, \overline{\boldsymbol{d}}_{h}\right) \\
+\frac{\gamma}{\varepsilon^{2}}\left(\left(\left|\boldsymbol{d}_{h}^{n}\right|^{2}-1\right) \boldsymbol{d}_{h}^{n+1, j}, \overline{\boldsymbol{d}}_{h}\right)=0 .
\end{gathered}
$$

It is proved the convergence of $\boldsymbol{d}_{h}^{n+1, j}$ to $\boldsymbol{d}_{h}^{n+1}$ in the $L^{2}$ norm, but it requires the relation $\gamma k / \varepsilon^{2}<1$ between the time and penalty parameter. In any case, for large scale problems and reasonably large time step sizes (e.g. ten times the explicit time step size), the assembling of the linear matrix is almost negligible compared to the solver time, and so, it is not clear the computational gain of this approach. On top of that, the iterations introduced over the $\boldsymbol{d}$ system are simple Richardson iterations, and so, it is expected to have a worse convergence than a Krylov-based solver.

\subsection{Mixed Methods for the Ginzburg-Landau Problem}

\subsubsection{Using $\boldsymbol{w}=\nabla \boldsymbol{d}$ as an Auxiliary Variable}

In a second work [45], Liu and Walkington avoided using Hermite finite elements for the approximation of the director equation in problem (11). The key idea is to introduce the auxiliary variable $\boldsymbol{w}=\nabla \boldsymbol{d}$ which allows one to formulate the director equation in the framework of mixed methods. Then, (35) can be written as finding $(\boldsymbol{u}(t), p(t), \boldsymbol{d}(t), \boldsymbol{w}(t)) \in \boldsymbol{H}_{0}^{1}(\Omega) \times L_{0}^{2}(\Omega) \times \boldsymbol{H}^{1}(\Omega) \times$
$\boldsymbol{H}(\operatorname{div}, \Omega)$ such that

$\left\{\begin{array}{l}\left(\boldsymbol{u}_{t}, \boldsymbol{v}\right)+c(\boldsymbol{u}, \boldsymbol{u}, \boldsymbol{v})+v a(\boldsymbol{u}, \boldsymbol{v}) \\ \quad+b_{u}(p, \boldsymbol{v})+\lambda c(\boldsymbol{u}, \boldsymbol{d}, \nabla \cdot \boldsymbol{w})=0, \\ b_{u}(p, \boldsymbol{u})=0, \\ \quad\left(\partial_{t} \boldsymbol{w}, \overline{\boldsymbol{w}}\right)-c(\boldsymbol{u}, \boldsymbol{d}, \nabla \cdot \overline{\boldsymbol{w}}) \\ \quad+\gamma(\nabla \cdot \boldsymbol{w}, \nabla \cdot \overline{\boldsymbol{w}})-\gamma\left(\boldsymbol{f}_{\varepsilon}(\boldsymbol{d}), \nabla \cdot \overline{\boldsymbol{w}}\right)=0, \\ a(\boldsymbol{d}, \overline{\boldsymbol{d}})+(\nabla \cdot \boldsymbol{w}, \overline{\boldsymbol{d}})=0,\end{array}\right.$

for all $(\overline{\boldsymbol{v}}, \bar{p}, \overline{\boldsymbol{d}}, \overline{\boldsymbol{w}}) \in H_{0}^{1}(\Omega) \times L_{0}^{2}(\Omega) \times H^{1}(\Omega) \times H(\operatorname{div}, \Omega)$. Note that $\Delta \boldsymbol{d}=\nabla \cdot \boldsymbol{w}$, which will be the extra equation to compute $\boldsymbol{d}$, and the elastic stress tensor is written as $(\nabla \boldsymbol{d})^{t} \nabla \cdot \boldsymbol{w}$ rather than $\boldsymbol{w}^{t} \nabla \cdot \boldsymbol{w}$; the convective term in the director equation remains the same $(\boldsymbol{u} \cdot \nabla) \boldsymbol{d}=(\nabla \boldsymbol{d})^{t} \boldsymbol{w}$, rather than $\boldsymbol{w}^{t} \boldsymbol{u}$. This is an important issue when obtaining error estimates, because one would need to establish high order norms for $\boldsymbol{w}$, e.g. an $\boldsymbol{L}^{4}$ norm, as used in [45]. However, this extra regularity is easier to obtain from the elliptic equation (42) $)_{4}$. Let us stress that $\boldsymbol{w}$ has $d^{2}$ components, where $d$ is the dimension space.

A fully discrete scheme to approximate (42) is used in [45], which is implicit with respect to time: given $\left(\boldsymbol{u}_{h}^{n}, \boldsymbol{d}_{h}^{n}\right)$, compute $\left(\boldsymbol{u}_{h}^{n+1}, p_{h}^{n+1}, \boldsymbol{w}_{h}^{n+1}, \boldsymbol{d}_{h}^{n+1}\right) \in\left(\boldsymbol{V}_{h}, P_{h}, \boldsymbol{W}_{h}, \boldsymbol{D}_{h}\right)$ such that

$$
\begin{aligned}
& \quad\left(\delta_{t} \boldsymbol{u}_{h}^{n+1}, \overline{\boldsymbol{u}}_{h}\right)+\widetilde{c}\left(\boldsymbol{u}_{h}^{n+1}, \boldsymbol{u}_{h}^{n+1}, \overline{\boldsymbol{u}}_{h}\right)+v a\left(\boldsymbol{u}^{n+1}, \overline{\boldsymbol{u}}_{h}\right) \\
& \quad-b_{u}\left(p_{h}^{n+1}, \overline{\boldsymbol{u}}_{h}\right)+\lambda c\left(\overline{\boldsymbol{u}}_{h}, \nabla \boldsymbol{d}_{h}^{n+1}, \nabla \cdot \boldsymbol{w}_{h}^{n+1}\right)=0, \\
& b_{u}\left(\bar{p}_{h}, \boldsymbol{u}_{h}^{n+1}\right)=0, \\
& \left(\delta_{t} \boldsymbol{w}_{h}^{n+1}, \overline{\boldsymbol{w}}_{h}\right)-c\left(\boldsymbol{u}_{h}^{n+1}, \boldsymbol{d}_{h}^{n+1}, \nabla \cdot \overline{\boldsymbol{w}}_{h}\right) \\
& \quad+\gamma\left(\nabla \cdot \boldsymbol{w}_{h}^{n+1}-\boldsymbol{f}_{\varepsilon}\left(\boldsymbol{d}_{h}^{n+1}\right), \nabla \cdot \overline{\boldsymbol{w}}_{h}\right)=0, \\
& a\left(\boldsymbol{d}_{h}^{n+1}, \overline{\boldsymbol{d}}_{h}\right)-\left(\nabla \cdot \boldsymbol{w}_{h}^{n+1}, \overline{\boldsymbol{d}}_{h}\right)=0,
\end{aligned}
$$

for all $\left(\overline{\boldsymbol{u}}_{h}, \bar{p}_{h}, \overline{\boldsymbol{w}}_{h}, \overline{\boldsymbol{d}}_{h}\right) \in \boldsymbol{V}_{h} \times P_{h} \times \boldsymbol{W}_{h} \times \boldsymbol{D}_{h}$, where $\boldsymbol{W}_{h}$ is an finite element approximation of $\boldsymbol{H}(\Omega$, div), e.g. the Raviart-Thomas [56] or the BDFM finite element [9].

The introduction of $\boldsymbol{w}$ allows to compute the director field and its first derivatives in an independent manner to keep an energy estimate similar to (37). However, the price to pay in terms of CPU cost is too large to be acceptable. The auxiliary unknown introduces four new components to be computed in dimension two, and nine in three dimensions, more than the number of unknowns of the original problem! Moreover, such a scheme is nonlinear, and some sort of iterative method must be performed at every time step.

The solvability of scheme (43) by Brouwer fixed point argument is subject to satisfying $k=\mathcal{O}\left(e^{-1 / \varepsilon^{2}}\right)$, which requires a very small time step for moderate values of $\varepsilon$. That restriction is a consequence of the energy estimate that scheme (43) provides, which is the same as for scheme (36), 
but expressed in terms of $\boldsymbol{w}$ as follows:

$$
\begin{aligned}
& \frac{1}{2}\left\|\boldsymbol{u}_{h}^{n+1}\right\|_{L^{2}(\Omega)}^{2}+\frac{\lambda}{2}\left\|\boldsymbol{w}_{h}^{n+1}\right\|_{L^{2}(\Omega)}^{2} \\
& \quad+k \sum_{m=0}^{n}\left(v\left\|\nabla \boldsymbol{u}_{h}^{m+1}\right\|_{L^{2}(\Omega)}^{2}+\frac{\lambda}{2} \gamma\left\|\nabla \cdot \boldsymbol{w}_{h}^{m+1}\right\|_{L^{2}(\Omega)}^{2}\right) \\
& \leq C \exp \left((n+1) k / \varepsilon^{2}\right) \\
& \quad \times\left(\frac{1}{2}\left\|\boldsymbol{u}_{h}^{0}\right\|_{L^{2}(\Omega)}^{2}+\frac{\lambda}{2}\left\|\boldsymbol{w}_{h}^{0}\right\|_{L^{2}(\Omega)}^{2}\right)
\end{aligned}
$$

for all $n$,

where the constant $C$ is a constant independent of $\varepsilon$. This bound blows up exponentially with $\varepsilon^{-2}$.

The hypotheses of regularity for a solution $(\boldsymbol{u}, p, \boldsymbol{d})$ of problem (11) are:

$\boldsymbol{u} \in C\left(0, T ; \boldsymbol{H}_{0}^{2}(\Omega)\right), \quad \partial_{t} \boldsymbol{u} \in L^{2}\left(0, T ; \boldsymbol{L}^{2}(\Omega)\right)$,

$\partial_{t t} \boldsymbol{u} \in L^{2}\left(0, T ; \boldsymbol{H}^{-1}(\Omega)\right), \quad p \in C\left(0, T ; H^{1}(\Omega)\right)$

and

$\boldsymbol{d} \in C\left(0, T ; \boldsymbol{W}^{2,4}(\Omega)\right), \quad \partial_{t} \boldsymbol{d} \in L^{2}\left(0, T ; \boldsymbol{H}^{1}(\Omega)\right)$,

$\partial_{t t} \boldsymbol{d} \in L^{2}\left(0, T ; \boldsymbol{L}^{2}(\Omega)\right)$.

In addition, $\boldsymbol{w} \in C\left(0, T ; \boldsymbol{H}^{2}(\Omega)\right)$. Then the following error estimates hold:

$$
\begin{gathered}
\left\|\boldsymbol{u}_{h}^{n}-\boldsymbol{u}\left(t_{n}\right)\right\|_{L^{2}(\Omega)}^{2}+\left\|\boldsymbol{w}_{h}^{n}-\nabla \boldsymbol{d}\left(t_{n}\right)\right\|_{L^{2}(\Omega)}^{2} \\
\quad+k \sum_{n=0}^{N}\left(\left\|\boldsymbol{u}_{h}^{n}-\boldsymbol{u}\left(t_{n}\right)\right\|_{H^{1}(\Omega)}^{2}\right. \\
\left.\quad+\left\|\nabla \cdot \boldsymbol{w}_{h}^{n}-\Delta \boldsymbol{d}\left(t_{n}\right)\right\|_{L^{2}(\Omega)}\right) \\
\leq C\left(k^{2}+h^{2}\right) \quad \text { for all } n .
\end{gathered}
$$

As for scheme (36), the regularity required for $\partial_{t t} \boldsymbol{u} \in$ $L^{2}\left(0, T ; \boldsymbol{H}^{-1}(\Omega)\right)$ needs a compatibility condition at $t=0$ to be satisfied (see [31]).

We would like to mention that this regularity assumption about the director vector to be approximated requires the domain $\Omega$ to hold (34) for the Neumann problem (33) with $r=4$; therefore a restriction on the angles of the boundary must be imposed.

\subsubsection{Using $\boldsymbol{w}=-\Delta \boldsymbol{d}$ as an Auxiliary Variable}

In order to avoid the large number of extra degrees of freedom and the nonlinearity of the numerical schemes above, Girault and Guillén-González [23] considered instead the auxiliary variable $-\boldsymbol{\Delta} \boldsymbol{d}$, constructing a fully discrete mixed scheme for (11) which is totally coupled but linear, unconditionally stable and convergent towards (11). Here we present a slight adaptation of the scheme given in [23] for the Neumann boundary condition.

Given $\left(\boldsymbol{u}_{h}^{n}, \boldsymbol{d}_{h}^{n}\right) \in\left(\boldsymbol{V}_{h}, \boldsymbol{D}_{h}\right)$, seek $\left(\boldsymbol{u}_{h}^{n+1}, p_{h}^{n+1}, \boldsymbol{w}_{h}^{n+1}\right.$, $\left.\boldsymbol{d}_{h}^{n+1}\right) \in \boldsymbol{V}_{h} \times P_{h} \times \boldsymbol{D}_{h} \times \boldsymbol{W}_{h}$ the solution of the linear algebraic system:

$$
\begin{aligned}
& \left(\delta_{t} \boldsymbol{u}_{h}^{n+1}, \overline{\boldsymbol{u}}_{h}\right)+\widetilde{c}\left(\boldsymbol{u}_{h}^{n}, \boldsymbol{u}_{h}^{n+1}, \overline{\boldsymbol{u}}_{h}\right)+v a\left(\boldsymbol{u}^{n+1}, \overline{\boldsymbol{u}}_{h}\right) \\
& \quad-b_{u}\left(p_{h}^{n+1}, \overline{\boldsymbol{u}}_{h}\right)-\lambda c\left(\overline{\boldsymbol{u}}_{h}, \boldsymbol{d}_{h}^{n}, \boldsymbol{w}_{h}^{n+1}\right)=0 \\
& b_{u}\left(\bar{p}_{h}, \boldsymbol{u}_{h}^{n+1}\right)=0 \\
& \left(\delta_{t} \boldsymbol{d}_{h}^{n+1}, \overline{\boldsymbol{w}}_{h}\right)+c\left(\boldsymbol{u}_{h}^{n+1}, \boldsymbol{d}_{h}^{n}, \overline{\boldsymbol{w}}_{h}\right) \\
& \quad+\gamma\left(\widetilde{\boldsymbol{f}}_{\varepsilon}\left(\boldsymbol{d}_{h}^{n}\right)+\boldsymbol{w}_{h}^{n+1}, \overline{\boldsymbol{w}}_{h}\right)=0 \\
& a\left(\boldsymbol{d}_{h}^{n+1}, \overline{\boldsymbol{d}}_{h}\right)-\left(\boldsymbol{w}_{h}^{n+1}, \overline{\boldsymbol{d}}_{h}\right)=0
\end{aligned}
$$

for all $\left(\overline{\boldsymbol{u}}_{h}, \bar{p}_{h}, \overline{\boldsymbol{w}}_{h}, \overline{\boldsymbol{d}}_{h}\right) \in \boldsymbol{V}_{h} \times P_{h} \times \boldsymbol{D}_{h} \times \boldsymbol{W}_{h}$ where $W_{h}$ is $\left(\mathcal{D}_{h}^{0}\right)^{d}$ and

$\tilde{\boldsymbol{f}}_{\varepsilon}(\boldsymbol{d})= \begin{cases}\boldsymbol{f}_{\varepsilon}(\boldsymbol{d}) & \text { if }|\boldsymbol{d}| \leq 1, \\ \mathbf{0} & \text { otherwise }\end{cases}$

In [23], it was observed that $\widetilde{\boldsymbol{f}}_{\varepsilon}=\boldsymbol{f}_{\varepsilon}(T(\boldsymbol{d}))$, where

$T(\boldsymbol{d})= \begin{cases}\boldsymbol{d} & \text { if }|\boldsymbol{d}| \leq 1, \\ \frac{\boldsymbol{d}}{|\boldsymbol{d}|} & \text { otherwise. }\end{cases}$

This truncation is impracticable from a numerical point of view. Therefore, a way to perform a tractable truncation is

$\widetilde{\boldsymbol{f}}_{\varepsilon}^{h}(\boldsymbol{d})=\frac{1}{\varepsilon^{2}}\left(T\left(P_{0}\left(|\boldsymbol{d}|^{2}\right)\right)-1\right) \boldsymbol{d}$,

where $P_{0}$ is the $L^{2}$ orthogonal projection onto $\boldsymbol{W}_{h}$; it implies a loop on the triangles.

After a slight adaptation of the stability proof in [23] for the Neumann boundary condition, we can prove that system (44) satisfies the following discrete energy law:

$$
\begin{aligned}
& \frac{1}{2}\left\|\boldsymbol{u}_{h}^{n+1}\right\|_{L^{2}(\Omega)}^{2}+\frac{\lambda}{2}\left\|\nabla \boldsymbol{d}_{h}^{n+1}\right\|^{2} \\
& \quad+k \sum_{m=1}^{n}\left(v\left\|\nabla \boldsymbol{u}_{h}^{m}\right\|_{L^{2}(\Omega)}^{2}+\lambda \gamma\left\|\boldsymbol{w}_{h}^{m}\right\|_{L^{2}(\Omega)}^{2}\right) \\
& \leq\left(\frac{1}{2}\left\|\boldsymbol{u}_{h}^{0}\right\|^{2}+\frac{\lambda}{2}\left\|\nabla \boldsymbol{d}_{h}^{0}\right\|^{2}\right) \exp \left((n+1) k / \varepsilon^{2}\right) \text { for all } n
\end{aligned}
$$

which again blows up exponentially with $\varepsilon^{-2}$.

This scheme reduces the degrees of freedom from schemes (36) and (43), leading to smaller linear algebraic problems, with the corresponding saving in terms of CPU time and storage, and only involves a linear system per time step. Although it is not immediate, scheme (44) can sligthly be modified in such a way that it satisfies an energy estimate independent of $\varepsilon$, under some assumptions over the choice of the discrete spaces. Let us introduce the following assumptions: 
(S) Stability conditions:

$$
\lim _{(h, k, \varepsilon) \rightarrow 0} \frac{k}{h^{2} \varepsilon^{2}}=0 \quad \text { and } \quad \frac{h}{\varepsilon} \leq C .
$$

(C) Convergence conditions:

$$
\lim _{(h, \varepsilon) \rightarrow 0} \frac{h}{\varepsilon^{2}}=0
$$

(H) The discrete spaces $\left(\boldsymbol{V}_{h}, \boldsymbol{D}_{h}\right)$ hold:

$$
\left(\boldsymbol{V}_{h} \cdot \nabla\right) \boldsymbol{D}_{h} \subset \boldsymbol{W}_{h} \quad \text { and } \quad \boldsymbol{D}_{h} \subset \boldsymbol{W}_{h} \text {. }
$$

Hypothesis $(\mathrm{H})$ indicates that $\boldsymbol{W}_{h}$ must be a discontinuous finite element space consisting of polynomial functions of degree more than or equal to that of $\boldsymbol{D}_{h}$ and $\left(\boldsymbol{V}_{h} \cdot \nabla\right) \boldsymbol{D}_{h}$, i.e. the space $\left(\mathcal{D}_{h}^{2}\right)^{d}$.

Moreover, the stabilizing term $b_{u}\left(F_{\varepsilon}\left(\boldsymbol{d}_{h}^{n}\right), \overline{\boldsymbol{u}}_{h}\right)$, related to a potential, must be added and $\left(\boldsymbol{d}_{h}^{n+1}-\boldsymbol{d}_{h}^{n}, \overline{\boldsymbol{d}}_{h}\right)$, which introduces some numerical dissipation. Therefore, the modified algorithm reads as: given $\left(\boldsymbol{u}_{h}^{n}, \boldsymbol{d}_{h}^{n}\right) \in\left(\boldsymbol{V}_{h}, \boldsymbol{D}_{h}\right)$, seek $\left(\boldsymbol{u}_{h}^{n+1}, p_{h}^{n+1}, \boldsymbol{w}_{h}^{n+1}, \boldsymbol{d}_{h}^{n+1}\right) \in \boldsymbol{V}_{h}^{b} \times P_{h} \times \boldsymbol{D}_{h} \times \boldsymbol{W}_{h}$ the solution of the linear algebraic system:

$$
\begin{aligned}
& \left(\delta_{t} \boldsymbol{u}_{h}^{n+1}, \overline{\boldsymbol{u}}_{h}\right)+\widetilde{c}\left(\boldsymbol{u}_{h}^{n}, \boldsymbol{u}_{h}^{n+1}, \overline{\boldsymbol{u}}_{h}\right)+v a\left(\boldsymbol{u}^{n+1}, \overline{\boldsymbol{u}}_{h}\right) \\
& \quad+\lambda b_{u}\left(F_{\varepsilon}\left(\boldsymbol{d}_{h}^{n}\right), \overline{\boldsymbol{u}}_{h}\right)-b_{u}\left(p_{h}^{n+1}, \overline{\boldsymbol{u}}_{h}\right) \\
& \quad-\lambda c\left(\overline{\boldsymbol{u}}_{h}, \boldsymbol{d}_{h}^{n}, \boldsymbol{w}_{h}^{n+1}\right)=0, \\
& b_{u}\left(\bar{p}_{h}, \boldsymbol{u}_{h}^{n+1}\right)=0, \\
& \left(\delta_{t} \boldsymbol{d}_{h}^{n+1}, \overline{\boldsymbol{w}}_{h}\right)+c\left(\boldsymbol{u}_{h}^{n+1}, \boldsymbol{d}_{h}^{n}, \overline{\boldsymbol{w}}_{h}\right) \\
& \quad+\gamma\left(\boldsymbol{f}_{\varepsilon}\left(\boldsymbol{d}_{h}^{n}\right)+\boldsymbol{w}_{h}^{n+1}, \overline{\boldsymbol{w}}_{h}\right)=0, \\
& a\left(\boldsymbol{d}_{h}^{n+1}, \overline{\boldsymbol{d}}_{h}\right)+\left(\boldsymbol{d}_{h}^{n+1}-\boldsymbol{d}_{h}^{n}, \overline{\boldsymbol{d}}_{h}\right)-\left(\boldsymbol{w}_{h}^{n+1}, \overline{\boldsymbol{d}}_{h}\right)=0 .
\end{aligned}
$$

The key pass to get the energy estimates independent of $\varepsilon$ for scheme (44) is taken form [29], which is based on an induction argument on the time step. Firstly, one obtains a discrete version of (12) at time $t_{n+1}$ by assuming that we have a control of the discrete kinetic, elastic and penalty energy at time $t_{n}$. The following proof is in fact new, since the bounds in [23] for the original scheme (without the previous modifications) blow up exponentially with $\varepsilon^{-2}$.

Lemma 3 Suppose that there exists a constant $C_{d}>0$ independent of $h, k$ and, $\varepsilon$ such that

$$
\left\|\boldsymbol{u}_{h}^{n}\right\|_{L^{2}(\Omega)}^{2}+\lambda\left\|\nabla \boldsymbol{d}_{h}^{n}\right\|_{L^{2}(\Omega)}^{2}+2 \lambda \int_{\Omega} F_{\varepsilon}\left(\boldsymbol{d}_{h}^{n}\right) \leq C_{d}
$$

Then there exist $h_{0}>0, k_{0}>0$, and $\varepsilon_{0}>0$ such that for all $h \leq h_{0}, k \leq k_{0}$, and $\varepsilon \leq \varepsilon_{0}$ satisfying hypothesis (S), the corresponding solution $\left(\boldsymbol{u}_{h}^{n+1}, \boldsymbol{d}_{h}^{n+1}, \boldsymbol{w}_{h}^{n+1}\right)$ of the discrete problem (47) satisfies the following inequality:

$$
\left\{\begin{array}{l}
\left(\left\|\boldsymbol{u}_{h}^{n+1}\right\|_{L^{2}(\Omega)}^{2}-\left\|\boldsymbol{u}_{h}^{n}\right\|_{L^{2}(\Omega)}^{2}\right)+\lambda\left(\left\|\nabla \boldsymbol{d}_{h}^{n+1}\right\|_{L^{2}(\Omega)}^{2}\right. \\
\left.\quad-\left\|\nabla \boldsymbol{d}_{h}^{n}\right\|_{L^{2}(\Omega)}^{2}\right)+2 \lambda \int_{\Omega}\left(F_{\varepsilon}\left(\boldsymbol{d}_{h}^{n+1}\right)-F_{\varepsilon}\left(\boldsymbol{d}_{h}^{n}\right)\right) \\
\quad+k\left(v\left\|\nabla \boldsymbol{u}_{h}^{n+1}\right\|_{L^{2}(\Omega)}^{2}\right. \\
\left.\quad+\lambda \gamma\left\|P_{W_{h}}\left(\boldsymbol{f}_{\varepsilon}\left(\boldsymbol{d}_{h}^{n}\right)\right)+\boldsymbol{w}_{h}^{n+1}\right\|_{L^{2}(\Omega)}^{2}\right) \leq 0,
\end{array}\right.
$$

where $P_{W_{h}}$ indicates the $L^{2}(\Omega)$-orthogonal projection onto $\boldsymbol{W}_{h}$.

Proof Take $\overline{\boldsymbol{u}}_{h}=2 k \boldsymbol{u}_{h}^{n+1}$ in (47) 1 and $\bar{p}_{h}=p_{h}^{n+1}$ in (47) 2 . Then, the term $b_{u}\left(p_{h}^{n+1}, \boldsymbol{u}_{h}^{n+1}\right)$ vanishes. Thus, the identity $(a-b, 2 a)=|a|^{2}-|b|^{2}+|a-b|^{2}$ provides

$$
\begin{aligned}
& \left\|\boldsymbol{u}_{h}^{n+1}\right\|_{L^{2}(\Omega)}^{2}-\left\|\boldsymbol{u}_{h}^{n}\right\|_{L^{2}(\Omega)}^{2}+\left\|\boldsymbol{u}_{h}^{n+1}-\boldsymbol{u}_{h}^{n}\right\|_{L^{2}(\Omega)}^{2} \\
& \quad+2 \nu k\left\|\nabla \boldsymbol{u}_{h}^{n+1}\right\|_{L^{2}(\Omega)}^{2}+2 \lambda k b_{u}\left(F_{\varepsilon}\left(\boldsymbol{d}_{h}^{n}\right), \boldsymbol{u}_{h}^{n+1}\right) \\
& \quad-2 \lambda k c\left(\boldsymbol{u}_{h}^{n+1}, \boldsymbol{d}_{h}^{n}, \boldsymbol{w}_{h}^{n+1}\right)=0 .
\end{aligned}
$$

On the other hand, consider $\overline{\boldsymbol{w}}_{h}=2 \lambda k\left(\boldsymbol{w}_{h}^{n+1}+P_{W_{h}}\left(\boldsymbol{f}_{\varepsilon}\left(\boldsymbol{d}_{h}^{n}\right)\right)\right)$ in (47) 3 and $\overline{\boldsymbol{d}}_{h}=2 \lambda\left(\boldsymbol{d}_{h}^{n+1}-\boldsymbol{d}_{h}^{n}\right)$ in (47) 4 . Next, using the fact that $\left(\boldsymbol{u}_{h}^{n+1} \cdot \nabla\right) \boldsymbol{d}_{h}^{n} \in \boldsymbol{W}_{h}$ and $\boldsymbol{d}_{h}^{n+1}-\boldsymbol{d}_{h}^{n} \in \boldsymbol{W}_{h}$ due to $(H)$, we get

$$
\begin{aligned}
\lambda( & \left.\left\|\nabla \boldsymbol{d}_{h}^{n+1}\right\|_{L^{2}(\Omega)}^{2}-\left\|\nabla \boldsymbol{d}_{h}^{n}\right\|_{L^{2}(\Omega)}^{2}+\left\|\boldsymbol{d}_{h}^{n+1}-\boldsymbol{d}_{h^{n}}^{n}\right\|_{H^{1}(\Omega)}^{2}\right) \\
& +2 \lambda\left(\boldsymbol{d}_{h}^{n+1}-\boldsymbol{d}_{h}^{n}, \boldsymbol{f}_{\varepsilon}\left(\boldsymbol{d}_{h}^{n}\right)\right) \\
& +2 \lambda k c\left(\boldsymbol{u}_{h}^{n+1}, \boldsymbol{d}_{h}^{n}, \boldsymbol{w}_{h}^{n+1}+\boldsymbol{f}_{\varepsilon}\left(\boldsymbol{d}_{h}^{n}\right)\right) \\
& +2 \lambda \gamma k\left\|P_{W_{h}}\left(\boldsymbol{f}_{\varepsilon}\left(\boldsymbol{d}_{h}^{n}\right)\right)+\boldsymbol{w}_{h}^{n+1}\right\|^{2}=0 .
\end{aligned}
$$

Now, if we add (50) and (51) and use

$c\left(\boldsymbol{u}_{h}^{n+1}, \boldsymbol{d}_{h}^{n}, \boldsymbol{f}_{\varepsilon}\left(\boldsymbol{d}_{h}^{n}\right)\right)+b_{u}\left(F_{\varepsilon}\left(\boldsymbol{d}_{h}^{n}\right), \boldsymbol{u}_{h}^{n+1}\right)=0$,

we have

$$
\begin{aligned}
& \left(\left\|\boldsymbol{u}_{h}^{n+1}\right\|_{L^{2}(\Omega)}^{2}+\lambda\left\|\nabla \boldsymbol{d}_{h}^{n+1}\right\|_{L^{2}(\Omega)}^{2}\right) \\
& \quad-\left(\left\|\boldsymbol{u}_{h}^{n}\right\|_{L^{2}(\Omega)}^{2}+\lambda\left\|\nabla \boldsymbol{d}_{h}^{n}\right\|_{L^{2}(\Omega)}^{2}\right) \\
& \quad+\left(\left\|\boldsymbol{u}_{h}^{n+1}-\boldsymbol{u}_{h}^{n}\right\|_{L^{2}(\Omega)}^{2}+\lambda\left\|\nabla\left(\boldsymbol{d}_{h}^{n+1}-\boldsymbol{d}_{h}^{n}\right)\right\|_{L^{2}(\Omega)}^{2}\right. \\
& \left.\quad+2 \lambda\left\|\boldsymbol{d}_{h}^{n+1}-\boldsymbol{d}_{h}^{n}\right\|_{L^{2}(\Omega)}^{2}\right) \\
& \quad+2 k\left(v\left\|\nabla \boldsymbol{u}_{h}^{n+1}\right\|_{L^{2}(\Omega)}^{2}+\gamma \lambda \| \boldsymbol{w}_{h}^{n+1}\right. \\
& \left.\quad+P_{W_{h}}\left(\boldsymbol{f}_{\varepsilon}\left(\boldsymbol{d}_{h}^{n}\right)\right) \|_{L^{2}(\Omega)}^{2}\right) \\
& \quad+2 \lambda\left(\boldsymbol{d}_{h}^{n+1}-\boldsymbol{d}_{h}^{n}, \boldsymbol{f}_{\varepsilon}\left(\boldsymbol{d}_{h}^{n}\right)\right) \leq 0
\end{aligned}
$$


Next, we decompose the last term on the left-hand side of (52) as follows:

$$
\begin{aligned}
2 \lambda & \left(\boldsymbol{d}_{h}^{n+1}-\boldsymbol{d}_{h}^{n}, \boldsymbol{f}_{\varepsilon}\left(\boldsymbol{d}_{h}^{n}\right)\right) \\
= & \frac{2 \lambda}{\varepsilon^{2}}\left(\boldsymbol{d}_{h}^{n+1}-\boldsymbol{d}_{h}^{n},\left(\left|\boldsymbol{d}_{h}^{n+1}\right|^{2}-1\right) \boldsymbol{d}_{h}^{n}\right) \\
& \quad+\frac{2 \lambda}{\varepsilon^{2}}\left(\boldsymbol{d}_{h}^{n+1}-\boldsymbol{d}_{h}^{n},\left(\left|\boldsymbol{d}_{h}^{n}\right|^{2}-\left|\boldsymbol{d}_{h}^{n+1}\right|^{2}\right) \boldsymbol{d}_{h}^{n}\right) \\
:= & I_{1}-I_{2} .
\end{aligned}
$$

Rewriting $I_{1}$ as

$$
\begin{aligned}
I_{1}= & \frac{\lambda}{\varepsilon^{2}} \int_{\Omega}\left(\left|\boldsymbol{d}_{h}^{n+1}\right|^{2}-1\right)\left(\left|\boldsymbol{d}_{h}^{n+1}\right|^{2}-\left|\boldsymbol{d}_{h}^{n}\right|^{2}-\left|\boldsymbol{d}_{h}^{n+1}-\boldsymbol{d}_{h}^{n}\right|^{2}\right) \\
= & \frac{\lambda}{2 \varepsilon^{2}} \int_{\Omega}\left(\left(F_{\varepsilon}\left(\boldsymbol{d}_{h}^{n+1}\right)-F_{\varepsilon}\left(\boldsymbol{d}_{h}^{n}\right)+\left(\left|\boldsymbol{d}_{h}^{n+1}\right|^{2}-\left|\boldsymbol{d}_{h}^{n}\right|^{2}\right)^{2}\right)\right. \\
& +\frac{\lambda}{\varepsilon^{2}} \int_{\Omega}\left(1-\left|\boldsymbol{d}_{h}^{n+1}\right|^{2}\right)\left|\boldsymbol{d}_{h}^{n+1}-\boldsymbol{d}_{h}^{n}\right|^{2}
\end{aligned}
$$

and bounding $I_{2}$ as

$$
\begin{aligned}
I_{2} \leq & \frac{C}{\varepsilon^{2}}\left\|\boldsymbol{d}_{h}^{n}\right\|_{L^{\infty}(\Omega)}^{2}\left\|\boldsymbol{d}_{h}^{n+1}-\boldsymbol{d}_{h}^{n}\right\|_{L^{2}(\Omega)}^{2} \\
& +\frac{\lambda}{4 \varepsilon^{2}} \int_{\Omega}\left(\left|\boldsymbol{d}_{h}^{n+1}\right|^{2}-\left|\boldsymbol{d}_{h}^{n}\right|^{2}\right)^{2}
\end{aligned}
$$

we arrive at

$$
\left\{\begin{array}{l}
\left(\left\|\boldsymbol{u}_{h}^{n+1}\right\|_{L^{2}(\Omega)}^{2}+\lambda\left\|\nabla \boldsymbol{d}_{h}^{n+1}\right\|_{L^{2}(\Omega)}^{2}+2 \lambda \int_{\Omega} F_{\varepsilon}\left(\boldsymbol{d}_{h}^{n+1}\right)\right) \\
-\left(\left\|\boldsymbol{u}_{h}^{n}\right\|_{L^{2}(\Omega)}^{2}+\lambda\left\|\nabla \boldsymbol{d}_{h}^{n}\right\|_{L^{2}(\Omega)}^{2}+2 \lambda \int_{\Omega} F_{\varepsilon}\left(\boldsymbol{d}_{h}^{n}\right)\right) \\
+\left\|\boldsymbol{u}_{h}^{n+1}-\boldsymbol{u}_{h}^{n}\right\|_{L^{2}(\Omega)}^{2}+\lambda\left\|\nabla\left(\boldsymbol{d}_{h}^{n+1}-\boldsymbol{d}_{h}^{n}\right)\right\|_{L^{2}(\Omega)}^{2} \\
+2 \lambda\left\|\boldsymbol{d}_{h}^{n+1}-\boldsymbol{d}_{h}^{n}\right\|_{L^{2}(\Omega)}^{2} \\
\left.\quad+\frac{\lambda}{\varepsilon^{2}} \int_{\Omega}\left(\frac{1}{4}\left(\left|\boldsymbol{d}_{h}^{n+1}\right|^{2}-\left|\boldsymbol{d}_{h}^{n}\right|^{2}\right)^{2}\right)+\left|\boldsymbol{d}_{h}^{n+1}-\boldsymbol{d}_{h}^{n}\right|^{2}\right) \\
\quad+2 k\left(v\left\|\nabla \boldsymbol{u}_{h}^{n+1}\right\|_{L^{2}(\Omega)}^{2}+\lambda \gamma \| P_{W_{h}}\left(\boldsymbol{f}_{\varepsilon}\left(\boldsymbol{d}_{h}^{n}\right)\right)\right. \\
\left.\quad+\boldsymbol{w}_{h}^{n+1} \|_{L^{2}(\Omega)}^{2}\right) \\
\leq \\
\frac{C}{\varepsilon^{2}}\left(\left\|\boldsymbol{d}_{h}^{n}\right\|_{L^{\infty}(\Omega)}^{2}+\left\|\boldsymbol{d}_{h}^{n+1}\right\|_{L^{\infty}(\Omega)}^{2}\right) \\
\quad \times\left\|\boldsymbol{d}_{h}^{n+1}-\boldsymbol{d}_{h}^{n}\right\|_{L^{2}(\Omega)}^{2}:=I_{3} .
\end{array}\right.
$$

We next want to bound the term $\left\|\boldsymbol{d}_{h}^{n+1}-\boldsymbol{d}_{h}^{n}\right\|_{L^{2}(\Omega)}^{2}$ of $I_{3}$. Take as a test function $\overline{\boldsymbol{w}}_{h}=P_{W_{h}}(\overline{\boldsymbol{w}})$ with $\overline{\boldsymbol{w}} \in \boldsymbol{L}^{3}(\Omega)$ into $(47)_{3}$. Then, by a duality argument, we have

$$
\begin{aligned}
\left\|\delta_{t} \boldsymbol{d}_{h}^{n+1}\right\|_{L^{3 / 2}(\Omega)} \leq & \left\|\boldsymbol{u}_{h}^{n+1}\right\|_{L^{6}(\Omega)}\left\|\nabla \boldsymbol{d}_{h}^{n}\right\|_{L^{2}(\Omega)} \\
& +\gamma\left\|P_{W_{h}}\left(\boldsymbol{f}_{\varepsilon}\left(\boldsymbol{d}_{h}^{n}\right)\right)+\boldsymbol{w}_{h}^{n+1}\right\|_{L^{2}(\Omega)} . \\
\leq & C\left(\nu\left\|\nabla \boldsymbol{u}_{h}^{n+1}\right\|_{L^{2}(\Omega)}\right. \\
& \left.+\gamma \lambda\left\|P_{W_{h}}\left(\boldsymbol{f}_{\varepsilon}\left(\boldsymbol{d}_{h}^{n}\right)\right)+\boldsymbol{w}_{h}^{n+1}\right\|_{L^{2}(\Omega)}\right)
\end{aligned}
$$

where we have used in the last line hypothesis (48). Next, the term $I_{3}$ can be handled as

$$
\begin{aligned}
I_{3} & \leq \frac{C k^{2}}{\varepsilon^{2}}\left(\left\|\boldsymbol{d}_{h}^{n}\right\|_{L^{\infty}(\Omega)}^{2}+\left\|\boldsymbol{d}_{h}^{n+1}\right\|_{L^{\infty}(\Omega)}^{2}\right)\left\|\delta_{t} \boldsymbol{d}_{h}^{n+1}\right\|_{L^{2}(\Omega)}^{2} \\
& \leq \frac{C k^{2}}{h^{2} \varepsilon^{2}}\left(\left\|\boldsymbol{d}_{h}^{n}\right\|_{H^{1}(\Omega)}^{2}+\left\|\boldsymbol{d}_{h}^{n+1}\right\|_{H^{1}(\Omega)}^{2}\right)\left\|\delta_{t} \boldsymbol{d}_{h}^{n+1}\right\|_{L^{3 / 2}(\Omega)}^{2}
\end{aligned}
$$

where the inverse inequalities

$$
\left\|\overline{\boldsymbol{d}}_{h}\right\|_{L^{2}(\Omega)} \leq C h^{-1 / 2}\left\|\overline{\boldsymbol{d}}_{h}\right\|_{L^{3 / 2}(\Omega)}
$$

and

$\left\|\overline{\boldsymbol{d}}_{h}\right\|_{L^{\infty}(\Omega)} \leq C h^{-1 / 2}\left\|\overline{\boldsymbol{d}}_{h}\right\|_{H^{1}(\Omega)} \quad$ for all $\overline{\boldsymbol{d}}_{h} \in \boldsymbol{D}_{h}$

have been used. Then the bound of $I_{3}$ remains as

$$
\begin{aligned}
I_{3} \leq & \frac{C k}{h^{2} \varepsilon^{2}}\left(\left\|\boldsymbol{d}_{h}^{n+1}\right\|_{H^{1}(\Omega)}^{2}+\left\|\boldsymbol{d}_{h}^{n}\right\|_{H^{1}(\Omega)}^{2}\right) \\
& \times\left(k v\left\|\nabla \boldsymbol{u}_{h}^{n+1}\right\|_{L^{2}(\Omega)}^{2}+\lambda \gamma k \| P_{W_{h}}\left(\boldsymbol{f}_{\varepsilon}\left(\boldsymbol{d}_{h}^{n}\right)\right)\right. \\
& \left.+\boldsymbol{w}_{h}^{n+1} \|_{L^{2}(\Omega)}^{2}\right) .
\end{aligned}
$$

Our next goal is to bound $\left\|\boldsymbol{d}_{h}^{n+1}\right\|_{H^{1}(\Omega)}$ in terms of $\left\|\boldsymbol{d}_{h}^{n}\right\|_{H^{1}(\Omega)}$ and $\left\|\boldsymbol{u}_{h}^{n}\right\|_{L^{2}(\Omega)}$. We consider (52) rewritten as

$$
\begin{aligned}
\left(\| \boldsymbol{u}_{h}^{n+1}\right. & \left.\left\|_{L^{2}(\Omega)}^{2}+\lambda\right\| \boldsymbol{d}_{h}^{n+1} \|_{H^{1}(\Omega)}^{2}\right) \\
& +\left(\left\|\boldsymbol{u}_{h}^{n+1}-\boldsymbol{u}_{h}^{n}\right\|_{L^{2}(\Omega)}^{2}+\lambda\left\|\boldsymbol{d}_{h}^{n+1}-\boldsymbol{d}_{h}^{n}\right\|_{H^{1}(\Omega)}^{2}\right) \\
& +2 k\left(v\left\|\nabla \boldsymbol{u}_{h}^{n+1}\right\|_{L^{2}(\Omega)}^{2}+\gamma \lambda \| \boldsymbol{w}_{h}^{n+1}\right. \\
& \left.+P_{W_{h}}\left(\boldsymbol{f}_{\varepsilon}\left(\boldsymbol{d}_{h}^{n}\right)\right) \|_{L^{2}(\Omega)}^{2}\right) \\
= & \left(\left\|\boldsymbol{u}_{h}^{n}\right\|_{L^{2}(\Omega)}^{2}+\lambda\left\|\boldsymbol{d}_{h}^{n}\right\|_{H^{1}(\Omega)}^{2}\right) \\
& \quad-2 \lambda\left(\boldsymbol{d}_{h}^{n+1}-\boldsymbol{d}_{h}^{n}, \boldsymbol{f}_{\varepsilon}\left(\boldsymbol{d}_{h}^{n}\right)\right)+2 \lambda\left(\boldsymbol{d}_{h}^{n}, \boldsymbol{d}_{h}^{n+1}-\boldsymbol{d}_{h}^{n}\right)
\end{aligned}
$$

The right-hand side of (55) can be estimated as

$$
\begin{aligned}
2 \lambda & \left(\boldsymbol{d}_{h}^{n+1}-\boldsymbol{d}_{h}^{n}, \boldsymbol{f}_{\varepsilon}\left(\boldsymbol{d}_{h}^{n}\right)\right) \\
\leq & C k\left\|\delta_{t} \boldsymbol{d}_{h}^{n+1}\right\|_{L^{3 / 2}(\Omega)} \frac{1}{h^{1 / 2}}\left\|\boldsymbol{f}_{\varepsilon}\left(\boldsymbol{d}_{h}^{n}\right)\right\|_{L^{2}(\Omega)} \\
\leq & \delta k\left\|\delta \boldsymbol{d}_{h}^{n+1}\right\|_{L^{3 / 2}(\Omega)}^{2}+C_{\delta} \frac{1}{h}\left\|\boldsymbol{f}_{\varepsilon}\left(\boldsymbol{d}_{h}^{n}\right)\right\|_{L^{2}(\Omega)}^{2} \\
\leq & \delta k\left\|\delta_{t} \boldsymbol{d}_{h}^{n+1}\right\|_{L^{3 / 2}(\Omega)}^{2}+C_{\delta} \frac{1}{h \varepsilon^{2}}\left\|\boldsymbol{d}_{h}^{n}\right\|_{L^{\infty}(\Omega)}^{2} F_{\varepsilon}\left(\boldsymbol{d}_{h}^{n}\right) \\
\leq & \delta k\left\|\delta_{t} \boldsymbol{d}_{h}^{n+1}\right\|_{L^{3 / 2}(\Omega)}^{2}+C_{\delta} \frac{1}{h^{2} \varepsilon^{2}} F_{\varepsilon}\left(\boldsymbol{d}_{h}^{n}\right)\left\|\boldsymbol{d}_{h}^{n}\right\|_{H^{1}(\Omega)}^{2} \\
\leq & C \delta k\left(v\left\|\nabla \boldsymbol{u}_{h}^{n+1}\right\|_{L^{2}(\Omega)}^{2}+\gamma \lambda \| \boldsymbol{w}_{h}^{n+1}\right. \\
& \left.+P_{W_{h}}\left(\boldsymbol{f}_{\varepsilon}\left(\boldsymbol{d}_{h}^{n}\right)\right) \|_{L^{2}(\Omega)}^{2}\right)+\frac{C_{\delta} k}{h^{2} \varepsilon^{2}} .
\end{aligned}
$$


The control of $\left\|\boldsymbol{d}_{h}^{n}\right\|_{H^{1}(\Omega)}$ in the fourth line of (56) comes from the inverse triangle inequality applied to $\int_{\Omega} F_{\varepsilon}\left(d_{h}^{n}\right) \leq$ $C_{d}$ to give

$$
\begin{aligned}
\left.\left.\left|\left\|\boldsymbol{d}_{h}^{n}\right\|_{L^{4}(\Omega)}^{2}-\right| \Omega\right|^{1 / 2}\right|^{2} & \leq\left\|\left|\boldsymbol{d}_{h}^{n}\right|^{2}-1\right\|_{L^{2}(\Omega)}^{2} \\
& =4 \varepsilon^{2} \int_{\Omega} F_{\varepsilon}\left(\boldsymbol{d}_{h}^{n}\right) \leq C_{d} \varepsilon^{2} .
\end{aligned}
$$

Hence, we have

$\left\|\boldsymbol{d}_{h}^{n}\right\|_{L^{4}(\Omega)}^{2} \leq C_{d}\left(\varepsilon+|\Omega|^{1 / 2}\right)$.

Thus, we complete the seminorm $\left\|\nabla \boldsymbol{d}_{h}^{n}\right\|^{2} \leq C_{d}$ in (48) to $\left\|\boldsymbol{d}_{h}^{n}\right\|_{H^{1}(\Omega)}^{2} \leq C$, where $C$ is independent of $h, k$, and $\varepsilon$. Therefore, from (56), we get, for $\delta$ small enough,

$$
\begin{aligned}
2 \lambda & \left(\boldsymbol{d}_{h}^{n+1}-\boldsymbol{d}_{h}^{n}, \boldsymbol{f}_{\varepsilon}\left(\boldsymbol{d}_{h}^{n}\right)\right) \\
\leq & k\left(v\left\|\nabla \boldsymbol{u}_{h}^{n+1}\right\|_{L^{2}(\Omega)}^{2}+\gamma \lambda \| \boldsymbol{w}_{h}^{n+1}\right. \\
& \left.+P_{W_{h}}\left(\boldsymbol{f}_{\varepsilon}\left(\boldsymbol{d}_{h}^{n}\right)\right) \|_{L^{2}(\Omega)}^{2}\right)+\frac{C k}{h^{2} \varepsilon^{2}} .
\end{aligned}
$$

It is easy to bound the last term on the right-hand side of (55) in a similar way:

$$
\begin{aligned}
2 \lambda & \left(\boldsymbol{d}_{h}^{n}, \boldsymbol{d}_{h}^{n+1}-\boldsymbol{d}_{h}^{n}\right) \\
& \leq 2 \lambda k\left\|\boldsymbol{d}_{h}^{n}\right\|_{L^{3}(\Omega)}\left\|\delta_{t} \boldsymbol{d}_{h}^{n+1}\right\|_{L^{3 / 2}(\Omega)} \\
& \leq \delta k\left\|\delta_{t} \boldsymbol{d}_{h}^{n+1}\right\|_{L^{3 / 2}(\Omega)}^{2}+C_{\delta} k .
\end{aligned}
$$

Thus, incorporating the bounds (57) and (58) to (55), one obtains

$$
\left\|\boldsymbol{d}_{h}^{n+1}\right\|_{H^{1}(\Omega)}^{2} \leq C\left(\left\|\boldsymbol{d}_{h}^{n}\right\|_{H^{1}(\Omega)}^{2}+\left\|\boldsymbol{u}_{h}^{n}\right\|_{L^{2}(\Omega)}^{2}+\frac{C k}{h^{2} \varepsilon^{2}}+C k\right)
$$

Therefore, using hypothesis (48) and $(S)$, we get the bound

$$
\left\|\boldsymbol{d}_{h}^{n+1}\right\|_{H^{1}(\Omega)}^{2} \leq C .
$$

Finally, applying (59) in (54), the term $I_{3}$ is bounded as

$$
\begin{aligned}
I_{3} \leq & C \frac{k}{h^{2} \varepsilon^{2}}\left(k \nu\left|\nabla \boldsymbol{u}_{h}^{n+1}\right|^{2}+\lambda \gamma k \mid P_{W_{h}}\left(\boldsymbol{f}_{\varepsilon}\left(\boldsymbol{d}_{h}^{n}\right)\right)\right. \\
& \left.+\left.\boldsymbol{w}_{h}^{n+1}\right|^{2}\right) .
\end{aligned}
$$

Using hypothesis $(S)$, we can select $\left(k_{0}, h_{0}, \varepsilon_{0}\right)$ such that for all $k \leq k_{0}, h \leq h_{0}$ and $\varepsilon \leq \varepsilon_{0}$,

$C \frac{k}{h^{2} \varepsilon^{2}} \leq 1$

and we arrive at

$$
\begin{aligned}
I_{3} \leq & k\left(v\left\|\nabla \boldsymbol{u}_{h}^{n+1}\right\|_{L^{2}(\Omega)}^{2}+\lambda \gamma \| P_{W_{h}}\left(\boldsymbol{f}_{\varepsilon}\left(\boldsymbol{d}_{h}^{n}\right)\right)\right. \\
& \left.+\boldsymbol{w}_{h}^{n+1} \|_{L^{2}(\Omega)}^{2}\right) .
\end{aligned}
$$

Therefore, we obtain inequality (49) using this estimate for $I_{3}$ in (53). It ends the proof.

In order to get stability estimates for scheme (47), we will need to assume the following estimates over the initial condition approximations $\boldsymbol{d}_{h}^{0}$ and $\boldsymbol{u}_{h}^{0}$ :

$\lambda\left\|\nabla \boldsymbol{d}_{h}^{0}\right\|_{L^{2}(\Omega)}^{2} \leq K_{1}, \quad\left\|\boldsymbol{u}_{h}^{0}\right\|_{L^{2}(\Omega)}^{2} \leq K_{2}$,

$2 \lambda \int_{\Omega} F_{\varepsilon}\left(d_{h}^{0}\right) d x \leq K_{3}$

where $K_{i}>0(i=1,2,3)$ are constants independent of $h$ and $k$.

The two first properties can be guaranteed e.g. considering $\boldsymbol{d}_{h}^{0}=I_{h}\left(\boldsymbol{d}_{0}\right)$ and $\boldsymbol{u}_{h}^{0}=J_{h}\left(\boldsymbol{u}_{0}\right)$ where $I_{h}$ and $J_{h}$ are interpolation operators being stable in $\boldsymbol{H}^{1}(\Omega) \cap \boldsymbol{L}^{\infty}(\Omega)$ and $L^{2}(\Omega)$, respectively, and having optimal error properties; for instance, the Scott-Zhang [52] or Clement [20] interpolant. It is not an easy task to construct initial approximations satisfying $\left|\boldsymbol{d}_{h}^{0}\right|=1$ (only constant linear finite element functions satisfy the restriction pointwise), so the only thing we can prove is a uniform bound with respect to $\varepsilon$ for $\int_{\Omega} F_{\varepsilon}\left(\boldsymbol{d}_{h}^{0}\right)$. Indeed, we have that the initial orientation of the liquid crystal molecules verifies the constraint $\left|\boldsymbol{d}_{0}\right|=1$. Therefore we can write

$$
\begin{aligned}
\int_{\Omega} F_{\varepsilon}\left(\boldsymbol{d}_{h}^{0}\right) & =\frac{1}{\varepsilon^{2}} \int_{\Omega}\left(\left|\boldsymbol{d}_{h}^{0}\right|^{2}-\left|\boldsymbol{d}_{0}\right|^{2}\right)^{2} \\
& =\frac{2}{\varepsilon^{2}} \int_{\Omega}\left(\boldsymbol{d}_{0}+\boldsymbol{d}_{h}^{0}, \boldsymbol{d}_{0}-\boldsymbol{d}_{h}^{0}\right)^{2} \\
& \leq \frac{1}{\varepsilon^{2}}\left\|\boldsymbol{d}_{0}+\boldsymbol{d}_{h}^{0}\right\|_{L^{\infty}(\Omega)}^{2}\left\|\boldsymbol{d}_{0}-\boldsymbol{d}_{h}^{0}\right\|_{L^{2}(\Omega)}^{2}
\end{aligned}
$$

Now, an optimal interpolation error implies that there is a positive constant $K_{3}$ such that

$2 \lambda \int_{\Omega} F_{\varepsilon}\left(\boldsymbol{d}_{h}^{0}\right) \leq C \frac{h^{2}}{\varepsilon^{2}} \leq K_{3}$,

which involves the new restriction for the parameters $h / \varepsilon \leq$ $K_{3}$ announced in $(C)$.

We now state the new results about the global-in-time stability for scheme (47):

Theorem 4 There exist $h_{0}, k_{0}$ and $\varepsilon_{0}$ so that for any $h \leq h_{0}$, $k \leq k_{0}$ and $\varepsilon \leq \varepsilon_{0}$ satisfying the stability condition $(\mathrm{S})$, the corresponding solutions of the discrete problem (44) satisfies the estimates:

$$
\begin{aligned}
& \text { (i) } \max _{0 \leq n \leq N}\left\|\boldsymbol{u}_{h}^{n}\right\|_{L^{2}(\Omega)} \leq C, \\
& \text { (ii) } k \sum_{n=0}^{N-1}\left\|\nabla \boldsymbol{u}_{h}^{n+1}\right\|_{L^{2}(\Omega)}^{2} \leq C,
\end{aligned}
$$


(iv) $\max _{0 \leq n \leq N}\left\|\boldsymbol{d}_{h}^{n}\right\|_{H^{1}(\Omega)} \leq C$,

(v) $k \sum_{n=0}^{N-1}\left\|P_{W_{h}}\left(\boldsymbol{f}_{\varepsilon}\left(\boldsymbol{d}_{h}^{n}\right)\right)+\boldsymbol{w}_{h}^{n+1}\right\|_{L^{2}(\Omega)}^{2} \leq C$,

(vii) $\max _{0 \leq n \leq N} \int_{\Omega} F_{\varepsilon}\left(\boldsymbol{d}_{h}^{n}\right) \leq C$,

where $C>0$ is independent of $(h, k, \varepsilon)$.

Proof It suffices to prove (49) for all $n=0, \ldots, N-1$. For this, we argue by induction on $n$. Let us define $C_{d}=$ $K_{1}+K_{2}+K_{3}$ with $K_{i}$ the bounds for the initial data. Then, in particular, $\left(\boldsymbol{u}_{h}^{0}, \boldsymbol{d}_{h}^{0}\right)$ satisfies the hypothesis of Lemma 3 for $n=0:\left\|\boldsymbol{u}_{h}^{0}\right\|_{L^{2}(\Omega)}^{2}+\lambda\left\|\nabla \boldsymbol{d}_{h}^{0}\right\|_{L^{2}(\Omega)}^{2}+2 \lambda \int_{\Omega} F_{\varepsilon}\left(\boldsymbol{d}_{h}^{0}\right) \leq C_{d}$, then (49) holds for $n=0$ (and, in particular, $\left\|\boldsymbol{u}_{h}^{1}\right\|_{L^{2}(\Omega)}^{2}+$ $\left.\lambda\left\|\nabla \boldsymbol{d}_{h}^{1}\right\|_{L^{2}(\Omega)}^{2}+2 \lambda \int_{\Omega} F_{\varepsilon}\left(\boldsymbol{d}_{h}^{1}\right) \leq C_{d}\right)$.

Now, we assume that $\left(\boldsymbol{u}_{h}^{s}, \boldsymbol{d}_{h}^{s}\right)$ holds (49) for $s=$ $1, \ldots, n-1$. Adding (49) for $s=1, \ldots, n-1$, one has

$$
\begin{aligned}
& \left\|\boldsymbol{u}_{h}^{n}\right\|_{L^{2}(\Omega)}^{2}+\lambda\left\|\nabla \boldsymbol{d}_{h}^{n}\right\|_{L^{2}(\Omega)}^{2}+2 \lambda \int_{\Omega} F_{\varepsilon}\left(\boldsymbol{d}_{h}^{n}\right) \\
& \quad \leq\left\|\boldsymbol{u}_{h}^{0}\right\|_{L^{2}(\Omega)}^{2}+\lambda\left\|\nabla \boldsymbol{d}_{h}^{0}\right\|_{L^{2}(\Omega)}^{2}+2 \lambda \int_{\Omega} F_{\varepsilon}\left(\boldsymbol{d}_{h}^{0}\right) \\
& \quad \leq K_{1}+K_{2}+K_{3}=C_{d},
\end{aligned}
$$

which implies from Lemma 3 that (49) holds for $n$.

The convergence for scheme (44) is demonstrated by two different ways: compactness and error estimates. The compactness for the discrete velocity is attained by estimating a discrete fractional time estimate for the velocity and director field. The line of argument is as follows. Add (44) 1 from $n=m$ to $m+r-1$ and take $\overline{\boldsymbol{u}}=\boldsymbol{u}_{h}^{m+r}-\boldsymbol{u}_{h}^{m}$ as a test function. Then, use adequately equation (44) ${ }_{2}$ and add from $m=1$ to $N-r$ to get

$$
\begin{aligned}
& k \sum_{m=1}^{N-r}\left\|\boldsymbol{u}_{h}^{m+r}-\boldsymbol{u}_{h}^{m}\right\|_{L^{2}(\Omega)}^{2} \\
& =-v k^{2} \sum_{m=1}^{N-r} \sum_{n=m}^{m+r} a\left(\boldsymbol{u}_{h}^{n+1}, \boldsymbol{u}_{h}^{m+r}-\boldsymbol{u}_{h}^{m}\right) \\
& \quad-k^{2} \sum_{m=1}^{N-r} \sum_{n=m}^{m+r} c_{h}\left(\boldsymbol{u}_{h}^{n}, \boldsymbol{u}_{h}^{n+1}, \boldsymbol{u}_{h}^{m+r}-\boldsymbol{u}_{h}^{m}\right) \\
& \quad-\lambda k^{2} \sum_{m=1}^{N-r} \sum_{n=m}^{m+r} c\left(\boldsymbol{u}_{h}^{m+r}-\boldsymbol{u}_{h}^{m}, \boldsymbol{d}_{h}^{n}, \boldsymbol{w}_{h}^{n+1}\right) .
\end{aligned}
$$

Let us now focus on how to control the last term. A discrete integration by parts and Sobolev's inequality leads to

$$
\begin{aligned}
\lambda k^{2} \sum_{m=1}^{N-r} \sum_{n=m}^{m+r} c\left(\boldsymbol{u}_{h}^{m+r}-\boldsymbol{u}_{h}^{m}, \boldsymbol{d}_{h}^{n}, \boldsymbol{w}_{h}^{n+1}\right) \\
\leq C(k r)^{1 / 2}\left(\sum_{n=1}^{N-1} k\left\|\boldsymbol{w}_{h}^{n+1}\right\|_{L^{2}(\Omega)}^{2}\right)^{1 / 2} \\
\quad \times\left(\sum_{n=1}^{N-1} k\left\|\nabla \boldsymbol{d}_{h}^{n}\right\|_{L^{3}(\Omega)}^{2}\right)^{1 / 2} \\
\quad \times\left(\sum_{m=1}^{N-r} k\left\|\nabla\left(\boldsymbol{u}_{h}^{m+r}-\boldsymbol{u}_{h}^{m}\right)\right\|_{L^{2}(\Omega)}^{2}\right)^{1 / 2} \\
\leq C(k r)^{1 / 2}
\end{aligned}
$$

Obviously, we need the control $k \sum_{n=1}^{N-1}\left\|\boldsymbol{d}_{h}^{n}\right\|_{W^{1,3}(\Omega)}^{2} \leq C$ to obtain (61), which takes advantage of $k \sum_{n=1}^{N-1}\left\|\boldsymbol{w}_{h}^{n}\right\|_{L^{2}(\Omega)}^{2} \leq$ $C$, proved in [23] by assuming $\partial \Omega$ to be only Lipschitz. Therefore, we arrive at

$$
\begin{aligned}
& \sum_{m=0}^{N-r}\left\|\boldsymbol{u}_{h}^{m}-\boldsymbol{u}_{h}^{m+r}\right\|_{L^{2}(\Omega)}^{2} d t \leq C(r k)^{1 / 2} \\
& \quad \text { for all } r=1, \ldots, N-1 .
\end{aligned}
$$

A similar bound for the discrete director can be obtained:

$\sum_{m=0}^{N-r}\left\|\boldsymbol{d}_{h}^{m+r}-\boldsymbol{d}_{h}^{m}\right\|_{H^{1}(\Omega)}^{2} d t \leq C(r k)^{1 / 2}$

$$
\text { for all } r=1, \ldots, N-1 \text {. }
$$

The expressions above, in term of time interpolations associated with the sequences $\left\{\boldsymbol{u}_{h}^{n}\right\}$ and $\left\{\boldsymbol{d}_{h}^{n}\right\}$, are written as

$\int_{0}^{T-\delta}\left\|\boldsymbol{u}_{h, k}^{r}(t+\delta)-\boldsymbol{u}_{h, k}^{r}(t)\right\|_{L^{2}(\Omega)}^{2} d t \leq C \delta^{1 / 2}$

for all $\delta \in(0, T)$,

$\int_{0}^{T-\delta}\left\|\boldsymbol{d}_{h k}^{r}(t+\delta)-\boldsymbol{d}_{h k}^{r}(t)\right\|_{H^{1}(\Omega)}^{2} d t \leq C \delta^{1 / 2}$

for all $\delta \in(0, T)$.

Next, a compactness result from [54] provides that the sequences $\left\{\boldsymbol{d}_{h, k}^{r}\right\}$ and $\left\{\boldsymbol{u}_{h, k}^{r}\right\}$ are compact in $L^{2}\left(0, T ; \boldsymbol{L}^{2}(\Omega)\right)$, which is extensible to $\left\{\boldsymbol{d}_{h, k}^{l}\right\}$ and $\left\{\boldsymbol{u}_{h, k}^{l}\right\}$. This strong convergence for $\left\{\boldsymbol{d}_{h, k}^{r}\right\}$ is not enough to pass to the limit in the elastic stress tensor of (44) 1 . Testing (44) 4 with $\overline{\boldsymbol{d}}=\boldsymbol{d}_{h}^{n+1}$ one can prove the strong convergence of $\left\{\nabla \boldsymbol{d}_{h, k}^{r}\right\}$ and $\left\{\nabla \boldsymbol{d}_{h, k}^{l}\right\}$ towards $\nabla \boldsymbol{d}$ in $L^{2}\left(0, T ; \boldsymbol{L}^{2}(\Omega)\right)$ as obtained in [23]. Therefore, one gets the existence of a global-in-time weak solution to (11) (see Definition 2). 
On the other hand, error estimates are obtained in [23]. Indeed, if the solution $(\boldsymbol{u}, p, \boldsymbol{d})$ to (11) has the following regularity:

$$
\begin{aligned}
& (\boldsymbol{u}, \boldsymbol{d}, \boldsymbol{w}) \in L^{2}\left(\boldsymbol{H}^{2}(\Omega) \times \boldsymbol{W}^{2,3}(\Omega) \times \boldsymbol{H}^{1}(\Omega)\right), \\
& \boldsymbol{u} \in L^{\infty}\left(\boldsymbol{W}^{1,3}(\Omega) \cap \boldsymbol{L}^{\infty}(\Omega)\right), \\
& p \in L^{2}\left(H^{1}(\Omega)\right),
\end{aligned}
$$$$
\left(\partial_{t} \boldsymbol{u}, \partial_{t} \boldsymbol{d}, \partial_{t} \boldsymbol{w}\right) \in L^{2}\left(\boldsymbol{H}^{1}(\Omega) \times \boldsymbol{H}^{2}(\Omega) \times \boldsymbol{W}^{1,6 / 5}(\Omega)\right),
$$

then scheme (44) satisfies the error estimates:

$$
\begin{aligned}
& \left\|\boldsymbol{e}_{\boldsymbol{u}}^{n}\right\|_{L^{2}(\Omega)}^{2}+\left\|\nabla \boldsymbol{e}_{\boldsymbol{d}}^{n}\right\|_{L^{2}(\Omega)}^{2} \\
& \quad+k \sum_{m=1}^{n+1}\left(\left\|\nabla \boldsymbol{e}_{\boldsymbol{u}}^{m}\right\|_{L^{2}(\Omega)}^{2}+\left\|\boldsymbol{e}_{\boldsymbol{w}}^{m}\right\|_{L^{2}(\Omega)}^{2}\right) \\
& \leq C\left(h^{2}+k^{2}\right) \quad \text { for all } n,
\end{aligned}
$$

where we recall that $e_{\boldsymbol{u}}^{n}:=\boldsymbol{u}_{h}^{n}-\boldsymbol{u}\left(t_{n}\right), e_{\boldsymbol{d}}^{n}:=\boldsymbol{u}_{h}^{n}-\boldsymbol{d}\left(t_{n}\right)$, and $e_{\boldsymbol{w}}^{n}:=\boldsymbol{w}_{h}^{n}-\boldsymbol{w}\left(t_{n}\right)$. These error estimates are derived by using an integral formulation for problem (11) which avoids to assume nonlocal compatibility conditions on the initial data [31]. Instead, the integral formulation requires more regularity for the time derivatives of the solution to be approximated.

Three iterative methods to decouple $\left(\boldsymbol{u}_{h}^{n+1}, p_{h}^{n+1}\right)$ from $\left(\boldsymbol{d}_{h}^{n+1}, \boldsymbol{w}_{h}^{n+1}\right)$ at each time step are also obtained for scheme (44) in [23]. For any $n \geq 0$, given $\boldsymbol{w}_{h}^{n}, \boldsymbol{d}_{h}^{n}, \boldsymbol{u}_{h}^{n}$ and $p_{h}^{n}$, these schemes compute $\boldsymbol{w}_{h}^{n+1}, \boldsymbol{d}_{h}^{n+1}, \boldsymbol{u}_{h}^{n+1}$ and $p_{h}^{n+1}$ as follows:

1. (a) Given $\boldsymbol{w}_{0}=\boldsymbol{w}_{h}^{n}$, if $n>1$, or $\boldsymbol{w}_{0}=-P_{W_{h}}\left(\Delta \boldsymbol{d}_{0}\right)$, if $n=1$, where $P_{W_{h}}$ is the $L^{2}$ orthogonal projection onto $\boldsymbol{W}_{h}$.

(b) For $i \geq 1$, known $\boldsymbol{w}_{i-1}$, compute $\left(\boldsymbol{u}_{i}, p_{i}\right) \in \boldsymbol{V}_{h} \times P_{h}$ such that

$$
\begin{aligned}
& \frac{1}{k}\left(\boldsymbol{u}_{i}-\boldsymbol{u}_{h}^{n}, \overline{\boldsymbol{u}}\right)+v a\left(\nabla \boldsymbol{u}_{i}, \nabla \boldsymbol{v}\right)-b_{u}\left(p_{i}, \nabla \cdot \boldsymbol{v}\right) \\
& \quad+c_{h}\left(\boldsymbol{u}_{h}^{n}, \boldsymbol{u}_{i}, \overline{\boldsymbol{u}}_{h}\right)=c\left(\overline{\boldsymbol{u}}_{h}, \boldsymbol{d}_{h}^{n} \boldsymbol{w}_{i-1}\right), \\
& b_{u}\left(\bar{p}_{h}, \boldsymbol{u}_{i}\right)=0 .
\end{aligned}
$$

(c) Next, compute $\left(\boldsymbol{w}_{i}, \boldsymbol{d}_{i}\right) \in \boldsymbol{W}_{h} \times \boldsymbol{D}_{h}$ the solution of:

$$
\begin{aligned}
& \frac{1}{k}\left(\boldsymbol{d}_{i}-\boldsymbol{d}_{h}^{n}, \overline{\boldsymbol{w}}_{h}\right)+\gamma\left(\boldsymbol{w}_{i}, \boldsymbol{w}_{h}\right) \\
& \quad=-c\left(\boldsymbol{u}_{i}, \boldsymbol{d}_{h}^{n}, \overline{\boldsymbol{w}}_{h}\right)-\gamma\left(\tilde{\boldsymbol{f}}_{\varepsilon}\left(\boldsymbol{d}_{h}^{n}\right), \overline{\boldsymbol{w}}_{h}\right), \\
& a\left(\boldsymbol{d}_{i}, \overline{\boldsymbol{d}}_{h}\right)-\left(\boldsymbol{w}_{i}, \overline{\boldsymbol{w}}_{h}\right)=0 .
\end{aligned}
$$

2. (a) Let $\boldsymbol{u}_{0}=\boldsymbol{u}_{h}^{n}$.

(b) Known $\boldsymbol{u}_{i-1}$, compute $\left(\boldsymbol{w}_{i}, \boldsymbol{d}_{i}\right) \in \boldsymbol{W}_{h} \times \boldsymbol{D}_{h}$, solution of:

$$
\begin{aligned}
& \frac{1}{k}\left(\boldsymbol{d}_{i}-\boldsymbol{d}_{h}^{n}, \overline{\boldsymbol{w}}_{h}\right)+\gamma\left(\boldsymbol{w}_{i}, \overline{\boldsymbol{w}}_{h}\right) \\
& \quad=-c\left(\boldsymbol{u}_{i-1}, \boldsymbol{d}_{h}^{n}, \overline{\boldsymbol{w}}_{h}\right)-\gamma\left(\widetilde{\boldsymbol{f}}_{\varepsilon}\left(\boldsymbol{d}_{h}^{n}\right), \overline{\boldsymbol{w}}_{h}\right), \\
& \left(\boldsymbol{d}_{i}, \overline{\boldsymbol{d}}_{h}\right)-\left(\boldsymbol{w}_{i}, \overline{\boldsymbol{d}}_{h}\right)=0 .
\end{aligned}
$$

(c) Next, compute $\left(\boldsymbol{u}_{i}, p_{i}\right) \in \boldsymbol{V}_{h} \times P_{h}$ such that

$$
\begin{aligned}
& \frac{1}{k}\left(\boldsymbol{u}_{i}-\boldsymbol{u}_{h}^{n}, \overline{\boldsymbol{u}}_{h}\right)+v a\left(\boldsymbol{u}_{i}, \overline{\boldsymbol{u}}_{h}\right)-b_{u}\left(p_{i}, \overline{\boldsymbol{u}}_{h}\right) \\
& \quad+c_{h}\left(\boldsymbol{u}_{h}^{n}, \boldsymbol{u}_{i}, \overline{\boldsymbol{u}}_{h}\right)=c\left(\overline{\boldsymbol{u}}_{h}, \boldsymbol{d}_{h}^{n}, \boldsymbol{w}_{i}\right) \\
& b_{u}\left(\bar{p}_{h}, \boldsymbol{u}_{i}\right)=0 .
\end{aligned}
$$

3. (a) Let $\boldsymbol{u}_{0}=\boldsymbol{u}_{h}^{n}$ and $\boldsymbol{w}_{0}=\boldsymbol{w}_{h}^{n}$, if $n>0$, or $\boldsymbol{w}_{0}=$ $-P_{W_{h}}\left(\Delta \boldsymbol{d}_{0}\right)$, if $n=1$.

(b) Known $\boldsymbol{u}_{i-1}$ and $\boldsymbol{w}_{i-1}$, compute in a parallel way

- $\left(\boldsymbol{w}_{i}, \boldsymbol{d}_{i}\right) \in \boldsymbol{W}_{h} \times \boldsymbol{D}_{h}$ such that

$$
\begin{aligned}
& \frac{1}{k}\left(\boldsymbol{d}_{i}-\boldsymbol{d}_{h}^{n}, \overline{\boldsymbol{w}}_{h}\right)+\gamma\left(\boldsymbol{w}_{i}, \overline{\boldsymbol{d}}_{h}\right) \\
& \quad=-c\left(\boldsymbol{u}_{i-1}, \boldsymbol{d}_{h}^{n}, \overline{\boldsymbol{w}}_{h}\right)-\gamma\left(\tilde{\boldsymbol{f}}\left(\boldsymbol{d}_{h}^{n}\right), \overline{\boldsymbol{w}}_{h}\right), \\
& a\left(\boldsymbol{d}_{i}, \overline{\boldsymbol{d}}_{h}\right)-\left(\boldsymbol{w}_{i}, \boldsymbol{d}_{h}\right)=0,
\end{aligned}
$$

- and $\left(\boldsymbol{u}_{i}, p_{i}\right) \in \boldsymbol{V}_{h} \times P_{h}$ such that

$$
\begin{aligned}
& \frac{1}{k}\left(\boldsymbol{u}_{i}-\boldsymbol{u}_{h}^{n}, \overline{\boldsymbol{u}}_{h}\right)+v a\left(\boldsymbol{u}_{i}, \overline{\boldsymbol{u}}_{h}\right)-b_{u}\left(p_{i}, \boldsymbol{u}_{h}\right) \\
& \quad=-c_{h}\left(\boldsymbol{u}_{h}^{n}, \boldsymbol{u}_{i-1}, \overline{\boldsymbol{u}}_{h}\right)+c\left(\overline{\boldsymbol{u}}_{h}, \boldsymbol{d}_{h}^{n}, \boldsymbol{w}_{i-1}\right), \\
& b_{u}\left(\bar{p}_{h}, \boldsymbol{u}_{i}\right)=0 .
\end{aligned}
$$

The convergence of these iterative methods is established under the condition $h^{2} \leq C k$, where $C>0$ is a constant depending on $\varepsilon$.

\subsubsection{Using $\boldsymbol{w}=-\Delta \boldsymbol{d}+\boldsymbol{f}_{\varepsilon}(\boldsymbol{d})$ as an Auxiliary Variable}

So far, the finite element schemes that have been presented to approximate a solution to the Ericksen-Leslie equations (3) by means of the Ginzburg-Landau equations (11) do not preserve a discrete version of the energy law (12). In fact, the only exception is the novel modification of (44), i.e. system (47). This is basically due to the fact that the nonlinear function $\boldsymbol{f}_{\varepsilon}(\boldsymbol{d})$ does not belong to the discrete space $\boldsymbol{D}_{h}$. In order to get such an energy law one must write the elastic tensor $\lambda \nabla \cdot\left((\nabla \boldsymbol{d})^{t} \nabla \boldsymbol{d}\right)$ in terms of the critical point equation $-\Delta \boldsymbol{d}+\boldsymbol{f}_{\varepsilon}(\boldsymbol{d})$ like

$$
\begin{aligned}
\lambda \nabla \cdot\left((\nabla \boldsymbol{d})^{t} \nabla \boldsymbol{d}\right)= & \lambda \nabla\left(\frac{1}{2}|\nabla \boldsymbol{d}|^{2}+F_{\varepsilon}(\boldsymbol{d})\right) \\
& -\lambda(\nabla \boldsymbol{d})^{t}\left(-\Delta \boldsymbol{d}+\boldsymbol{f}_{\varepsilon}(\boldsymbol{d})\right),
\end{aligned}
$$


as in $[8,29]$. Then, if one defines the variable $\boldsymbol{w}=$ $-\Delta d+f_{\varepsilon}(d)$, which represents the Euler-Lagrange equation related to the Ginzburg-Landau free energy functional $\int_{\Omega} \frac{1}{2}|\nabla \boldsymbol{d}|^{2}+F_{\varepsilon}(\boldsymbol{d})$, model (11) can be reformulated as

$$
\begin{cases}\partial_{t} \boldsymbol{u}+\boldsymbol{u} \cdot \nabla \boldsymbol{u}-v \Delta \boldsymbol{u}+\nabla p-\lambda(\nabla \boldsymbol{d})^{t} \boldsymbol{w}=\mathbf{0} & \text { in } Q, \\ \nabla \cdot \boldsymbol{u}=0 & \text { in } Q, \\ |\boldsymbol{d}| \leq 1, \partial_{t} \boldsymbol{d}+\boldsymbol{u} \cdot \nabla \boldsymbol{d}+\gamma \boldsymbol{w}=\mathbf{0} & \text { in } Q, \\ -\Delta \boldsymbol{d}+\boldsymbol{f}_{\varepsilon}(\boldsymbol{d})-\boldsymbol{w}=\mathbf{0} & \text { in } Q,\end{cases}
$$

where the pressure $p$ is modified by the potential function $p+\frac{\lambda}{2}|\nabla \boldsymbol{d}|^{2}+\lambda F_{\varepsilon}(\boldsymbol{d})$ (which is called again $p$ for simplicity).

The vector spaces where the weak formulation of problem (65) is well-posed are as follows: find $(\boldsymbol{u}(t), p(t)$, $\boldsymbol{d}(t), \boldsymbol{w}(t)) \in \boldsymbol{H}_{0}^{1}(\Omega) \times L_{0}^{2}(\Omega) \times \boldsymbol{H}^{1}(\Omega) \times \boldsymbol{L}^{2}(\Omega)$ such that

$$
\begin{aligned}
& \left(\partial_{t} \boldsymbol{u}, \overline{\boldsymbol{u}}\right)+c(\boldsymbol{u}, \boldsymbol{u}, \overline{\boldsymbol{u}})+v a(\boldsymbol{u}, \overline{\boldsymbol{u}}) \\
& \quad-b_{u}(p, \overline{\boldsymbol{u}})-\lambda c(\overline{\boldsymbol{u}}, \boldsymbol{d}, \boldsymbol{w})=0, \\
& b_{u}(\bar{p}, \boldsymbol{u})=0, \\
& \left(\partial_{t} \boldsymbol{d}, \overline{\boldsymbol{w}}\right)+c(\boldsymbol{u}, \boldsymbol{d}, \overline{\boldsymbol{w}})+\gamma(\boldsymbol{w}, \overline{\boldsymbol{w}})=0, \\
& a(\boldsymbol{d}, \overline{\boldsymbol{d}})+\left(\boldsymbol{f}_{\varepsilon}(\boldsymbol{d}), \overline{\boldsymbol{d}}\right)-(\boldsymbol{w}, \overline{\boldsymbol{d}})=0,
\end{aligned}
$$

for all $(\overline{\boldsymbol{u}}, \bar{p}, \overline{\boldsymbol{w}}, \overline{\boldsymbol{d}}) \in \boldsymbol{H}_{0}^{1}(\Omega) \times L_{0}^{2}(\Omega) \times \boldsymbol{L}^{2}(\Omega) \times \boldsymbol{H}^{1}(\Omega)$.

Two finite-element Euler time-stepping schemes [8, 29] have been developed to approximate (66), being both implicit for the linear terms and semi-implicit for the nonlinear ones. The main difference among them lies in the way of treating the time integration of $\boldsymbol{f}_{\varepsilon}$. Becker, Feng and Prohl proposed in [8] a fully implicit approximation while Guillén-González and Gutiérrez-Santacreu suggested a fully explicit one.

Thus, the scheme developed in [8] is expressed as follows. Let $\left(\boldsymbol{u}_{h}^{n}, \boldsymbol{d}_{h}^{n}\right) \in\left(\boldsymbol{V}_{h}, \boldsymbol{D}_{h}\right)$ be given, then find the solution $\left(\boldsymbol{u}_{h}^{n+1}, p_{h}^{n+1}, \boldsymbol{d}_{h}^{n+1}, \boldsymbol{w}_{h}^{n+1}\right) \in \boldsymbol{V}_{h} \times P_{h} \times \boldsymbol{D}_{h} \times \boldsymbol{W}_{h}$ of the nonlinear system:

$$
\left\{\begin{array}{l}
\left(\delta_{t} \boldsymbol{u}_{h}^{n+1}, \overline{\boldsymbol{u}}_{h}\right)+\widetilde{c}\left(\boldsymbol{u}_{h}^{n}, \boldsymbol{u}_{h}^{n+1}, \overline{\boldsymbol{u}}_{h}\right)+v a\left(\boldsymbol{u}_{h}^{n+1}, \overline{\boldsymbol{u}}_{h}\right) \\
\quad-b_{u}\left(p_{h}^{n+1}, \overline{\boldsymbol{u}}_{h}\right)-\lambda c\left(\overline{\boldsymbol{u}}_{h}, \boldsymbol{d}_{h}^{n}, \boldsymbol{w}_{h}^{n+1}\right)=0 \\
b_{u}\left(\bar{p}_{h}, \boldsymbol{u}_{h}^{n+1}\right)=0 \\
\left(\delta_{t} \boldsymbol{d}_{h}^{n+1}, \overline{\boldsymbol{w}}_{h}\right)+c\left(\boldsymbol{u}_{h}^{n+1}, \boldsymbol{d}_{h}^{n}, \overline{\boldsymbol{w}}_{h}\right)+\gamma\left(\boldsymbol{w}_{h}^{n+1}, \overline{\boldsymbol{w}}_{h}\right)=0 \\
a\left(\boldsymbol{d}_{h}^{n+1}, \overline{\boldsymbol{d}}_{h}\right)+\left(\boldsymbol{f}_{\varepsilon}\left(\boldsymbol{d}_{h}^{n+1}, \boldsymbol{d}_{h}^{n}\right), \overline{\boldsymbol{d}}_{h}\right)_{h}-\left(\boldsymbol{w}_{h}^{n+1}, \overline{\boldsymbol{d}}_{h}\right)=0
\end{array}\right.
$$

for all $\left(\overline{\boldsymbol{u}}_{h}, \bar{p}_{h}, \overline{\boldsymbol{w}}_{h}, \overline{\boldsymbol{d}}_{h}\right) \in \boldsymbol{V}_{h} \times P_{h} \times \boldsymbol{W}_{h} \times \boldsymbol{D}_{h}$, where $\boldsymbol{f}_{\varepsilon}\left(\boldsymbol{d}_{h}^{n+1}, \boldsymbol{d}_{h}^{n}\right)=\frac{1}{2 \varepsilon^{2}}\left|\boldsymbol{d}_{h}^{n+1}\right|^{2} \boldsymbol{d}_{h}^{n+1}-\boldsymbol{d}_{h}^{n}$. Here, a discrete inner product is used $(\cdot, \cdot)_{h}$, which is defined as follows. Let $\left\{\boldsymbol{\phi}_{a}: \boldsymbol{a} \in N_{h}\right\}$ denote the nodal basis associated the set of all nodes $\mathcal{N}_{h}=\left\{\boldsymbol{a}_{l}\right\}_{l \in L}$ of $\mathcal{T}_{h}$. Thus, the nodal interpolation operator $I_{D_{h}}: C(\bar{\Omega}) \rightarrow D_{h}$ is such that $I_{D_{h}} \boldsymbol{\psi}:=$ $\sum_{\boldsymbol{a} \in \mathcal{N}_{h}} \boldsymbol{\psi}(\boldsymbol{a}) \boldsymbol{\phi}_{\boldsymbol{a}}$. Then the discrete inner product $(\cdot, \cdot)_{h}$ is defined in the following way: for all $\Psi, \psi \in C(\bar{\Omega})$, one has

$$
(\boldsymbol{\Psi}, \boldsymbol{\psi})_{h}:=\int_{\Omega} I_{h}(\boldsymbol{\Psi} \cdot \boldsymbol{\psi})=\sum_{\boldsymbol{a} \in \mathcal{N}_{h}} \boldsymbol{\Psi}(\boldsymbol{a}) \cdot \boldsymbol{\psi}(\boldsymbol{a}) \int_{\Omega} \boldsymbol{\phi}_{\boldsymbol{a}}
$$

This discrete inner-product applied to the potential term $\left(\boldsymbol{f}_{\varepsilon}\left(\boldsymbol{d}_{h}^{n+1}, \boldsymbol{d}_{h}^{n}\right), \overline{\boldsymbol{d}}_{h}\right)_{h}$ produces a $1 / \varepsilon^{2}$-diagonal lumped mass matrix. A Newton method is considered in [8] to linearize the problem.

Scheme (67) provides a discrete energy law which mimics the continuous energy law (12):

$$
\begin{aligned}
& \frac{1}{2}\left\|\boldsymbol{u}_{h}^{n+1}\right\|_{L^{2}(\Omega)}^{2}+\frac{\lambda}{2}\left\|\nabla \boldsymbol{d}_{h}^{n+1}\right\|_{L^{2}(\Omega)}^{2}+\lambda \int_{\Omega} F_{\varepsilon}\left(\boldsymbol{d}_{h}^{n+1}\right) \\
& +k \sum_{m=1}^{n+1}\left(v\left\|\nabla \boldsymbol{u}_{h}^{m}\right\|_{L^{2}(\Omega)}^{2}+\lambda \gamma\left\|\boldsymbol{w}_{h}^{m}\right\|_{L^{2}(\Omega)}^{2}\right) \\
& \leq\left(\frac{1}{2}\left\|\boldsymbol{u}_{h}^{0}\right\|_{L^{2}(\Omega)}^{2}+\frac{\lambda}{2}\left\|\nabla \boldsymbol{d}_{h}^{0}\right\|_{L^{2}(\Omega)}^{2}\right. \\
& \left.+\int_{\Omega} F_{\varepsilon}\left(\boldsymbol{d}_{h}^{0}\right)\right) \text { for all } n .
\end{aligned}
$$

This scheme is unconditionally stable and convergent towards a measured-valued solution of (3). This convergence is attained in two steps; firstly, when the time and space discretization parameters go to zero, the convergence towards a weak solution of the penalized problem (65) is proved, and afterwards, when the penalty parameter $\varepsilon$ goes to zero, one arrives at a measure-valued solution of problem (3), where the elastic tensor $(\nabla \boldsymbol{d})^{t} \nabla \boldsymbol{d}$ only tends to a certain measure (see [39]).

The scheme presented in [29] was designed for nonhomogeneous Dirichlet boundary conditions. This scheme should be redesigned appropriately in order to guarantee stability for homogeneous Neumann boundary conditions. The renewed scheme preserves the time discretization of the penalty function in a fully explicit way. Then, the scheme becomes: given $\left(\boldsymbol{u}_{h}^{n}, \boldsymbol{d}_{h}^{n}\right) \in\left(\boldsymbol{V}_{h}, \boldsymbol{D}_{h}\right)$, find $\left(\boldsymbol{u}_{h}^{n+1}, p_{h}^{n+1}, \boldsymbol{d}_{h}^{n+1}, \boldsymbol{w}_{h}^{n+1}\right) \in \boldsymbol{V}_{h} \times P_{h} \times \boldsymbol{D}_{h} \times \boldsymbol{W}_{h}$ solving the finite element linear system:

$$
\left\{\begin{array}{l}
\left(\delta_{t} \boldsymbol{u}_{h}^{n+1}, \overline{\boldsymbol{u}}_{h}\right)+\widetilde{c}\left(\boldsymbol{u}_{h}^{n}, \boldsymbol{u}_{h}^{n+1}, \overline{\boldsymbol{u}}_{h}\right)+v a\left(\boldsymbol{u}_{h}^{n+1}, \overline{\boldsymbol{u}}_{h}\right) \\
\quad-b_{u}\left(p_{h}^{n+1}, \overline{\boldsymbol{u}}_{h}\right)-\lambda c\left(\overline{\boldsymbol{u}}_{h}, \boldsymbol{d}_{h}^{n}, \boldsymbol{w}_{h}^{n+1}\right)=0 \\
b_{u}\left(\bar{p}_{h}, \boldsymbol{u}_{h}^{n+1}\right)=0 \\
\left(\delta_{t} \boldsymbol{d}_{h}^{n+1}, \overline{\boldsymbol{w}}_{h}\right)+c\left(\boldsymbol{u}_{h}^{n+1}, \boldsymbol{d}_{h}^{n}, \overline{\boldsymbol{w}}_{h}\right)+\gamma\left(\boldsymbol{w}_{h}^{n+1}, \overline{\boldsymbol{w}}_{h}\right)=0 \\
a\left(\boldsymbol{d}_{h}^{n+1}, \overline{\boldsymbol{d}}_{h}\right)+\left(\boldsymbol{f}_{\varepsilon}\left(\boldsymbol{d}_{h}^{n}\right), \overline{\boldsymbol{d}}_{h}\right)_{h}-\left(\boldsymbol{w}_{h}^{n+1}, \overline{\boldsymbol{d}}_{h}\right)=0
\end{array}\right.
$$

for all $\left(\overline{\boldsymbol{u}}_{h}, \bar{p}_{h}, \overline{\boldsymbol{w}}_{h}, \overline{\boldsymbol{d}}_{h}\right) \in \boldsymbol{V}_{h} \times P_{h} \times \boldsymbol{W}_{h} \times \boldsymbol{D}_{h}$. 
Scheme (69) is conditionally stable in the terms of scheme (47) (assuming (S)) and convergent (assuming (C)) to a measure-valued solution, but this time the convergence is attained by making the mesh parameter $(h, k)$ and the penalty parameter $\varepsilon$ go to zero at the same time, what could be done for scheme (67) as well. Since the approximation of $\boldsymbol{f}_{\varepsilon}$ is explicit, the linear algebraic problem (69) does not depend on $\varepsilon$, which assures that the conditional number of the system at every time step is not affected by $\varepsilon$. However, the stability condition (S) implies that the time step $k$ must be quite small if the size of $\varepsilon$ is proportional to the space parameter $h$, but numerical experiences in [29] have demonstrated to be optimal.

The convergence of schemes (67) and (69) is obtained by means of compactness results. For the discrete director vector, it is easy to check that

$$
k \sum_{n=0}^{N-1}\left\|\delta_{t} \boldsymbol{d}_{h}^{n+1}\right\|_{L^{3 / 2}(\Omega)}^{2} \leq C,
$$

by choosing as a test function $\overline{\boldsymbol{w}}_{h}=P_{W_{h}} \overline{\boldsymbol{w}}$ in (67) or (69); we have also used the fact that $\boldsymbol{w} \in \boldsymbol{L}^{3}(\Omega)$ and the $L^{3}(\Omega)$ stability of the $L^{2}$ projection operator $P_{W_{h}}$ (see [15]). As a consequence of the energy estimates and a compactness result in [54], one gets the compactness of the sequence $\left\{\boldsymbol{d}_{h, k, \varepsilon}\right\}$ in $L^{q}\left(0, T ; \boldsymbol{L}^{r}(\Omega)\right)$ with $1 \leq r<6$ and $1 \leq q<\infty$, where $\boldsymbol{d}_{h, k, \varepsilon}$ is the linear piecewise continuous function such that $\boldsymbol{d}_{h, k, \varepsilon}\left(t_{n}\right)=\boldsymbol{d}_{h}^{n}$.

We think the way how the compactness for the discrete velocity in $L^{2}\left(0, T ; \boldsymbol{L}^{2}(\Omega)\right)$ is proved in [8] is not clear. From (68), the sequence of discrete velocities is bounded in $L^{\infty}\left(0, T ; \boldsymbol{L}^{2}(\Omega)\right) \cap L^{2}\left(0, T ; \boldsymbol{H}_{0}^{1}(\Omega)\right)$. Afterwards, by a duality argument, the discrete time derivative for the velocity is bounded in the dual space of $\boldsymbol{V} \cap \boldsymbol{H}^{2}(\Omega)$. Then the AubinLions compactness lemma is used. To apply this compactness result, one needs the embeddings $\boldsymbol{H}_{0}^{1}(\Omega) \stackrel{\text { compact }}{\mapsto}$ $\boldsymbol{L}^{2}(\Omega) \hookrightarrow\left(\boldsymbol{V} \cap \boldsymbol{H}^{2}(\Omega)\right)^{\prime}$, but the embedding from $\boldsymbol{L}^{2}(\Omega)$ into $\left(\boldsymbol{V} \cap \boldsymbol{H}^{2}(\Omega)\right)^{\prime}$ is not injective. Obtaining a compactness result for the discrete velocity turns out to be harder than for the Ginzburg-Landau problem (11) for a fixed $\varepsilon$, even for problems with a similar structure such as the densitydependent Navier-Stokes equations ([27, 46] and [28]).

Now we cannot prove the time fractional estimate

$$
\sum_{m=0}^{N-r}\left\|\boldsymbol{u}_{h}^{m}-\boldsymbol{u}_{h}^{m+r}\right\|_{L^{2}(\Omega)}^{2} d t \leq C(r k)^{1 / 2}
$$

for all $r=1, \ldots, N-1$

obtained in [23], since no control over $k \sum_{n=1}^{N-1}\left\|\boldsymbol{d}_{h}^{n}\right\|_{W^{1,3}(\Omega)}^{2}$ $\leq C$ is available with $C$ being independent of $\varepsilon$. Let us sketch the way of getting compactness for the discrete velocity in an appropriate way. Let

$\boldsymbol{X}_{h}=\left\{\overline{\boldsymbol{u}}_{h} \in \boldsymbol{V}_{h}: b_{u}\left(\bar{p}_{h}, \overline{\boldsymbol{u}}_{h}\right)=0 \forall \bar{p}_{h} \in P_{h}\right\}$ be the discrete divergence-free space associated with $\boldsymbol{V}_{h}$ and consider $A_{h}^{-1}: \boldsymbol{V}_{h} \rightarrow \boldsymbol{X}_{h}$ the inverse discrete Stokes operator defined as

$\left(\nabla A_{h}^{-1} \boldsymbol{u}_{h}, \nabla \boldsymbol{v}_{h}\right)=\left(\boldsymbol{u}_{h}, \boldsymbol{v}_{h}\right) \quad \forall \boldsymbol{v}_{h} \in \boldsymbol{X}_{h}$

Notice that (70) is well-defined owing to the inf-sup condition (30).

By multiplying (67) ${ }_{1}$ by $k^{2}$, summing for $n=m, \ldots$, $m-1+r$, setting $\overline{\boldsymbol{u}}_{h}=A_{h}^{-1}\left(\boldsymbol{u}_{h}^{m+r}-\boldsymbol{u}_{h}^{m}\right)$ as a test function in $(67)_{1}$, and summing for $m=0, \ldots, N-r$, we get

$$
\begin{aligned}
& k \sum_{m=0}^{N-r}\left|\nabla A_{h}^{-1}\left(\boldsymbol{u}_{h}^{m+r}-\boldsymbol{u}_{h}^{m}\right)\right|^{2} \\
& =-k^{2} \sum_{m=0}^{N-r} \sum_{n=m}^{m-1+r} c\left(\boldsymbol{u}_{h}^{n}, \boldsymbol{u}_{h}^{n+1}, A_{h}^{-1}\left(\boldsymbol{u}_{h}^{m+r}-\boldsymbol{u}_{h}^{m}\right)\right) \\
& \quad+\nu k^{2} \sum_{m=0}^{N-r} \sum_{n=m}^{m-1+r}\left(\nabla \boldsymbol{u}^{n+1}, \nabla A_{h}^{-1}\left(\boldsymbol{u}_{h}^{m+r}-\boldsymbol{u}_{h}^{m}\right)\right) \\
& \quad+\lambda k^{2} \sum_{m=0}^{N-r} \sum_{n=m}^{m-1+r}\left(\left(\nabla \boldsymbol{d}_{h}^{n}\right)^{t} \boldsymbol{w}_{h}^{n+1}, A_{h}^{-1}\left(\boldsymbol{u}_{h}^{m+r}-\boldsymbol{u}_{h}^{m}\right)\right) \\
& :=J_{1}+J_{2}+J_{3} .
\end{aligned}
$$

Let us only focus on how to estimate $J_{3}$

$$
\begin{aligned}
J_{3} & \leq C k^{2} \sum_{m=0}^{N-r} \sum_{n=m}^{m-1+r}\left|\nabla \boldsymbol{d}_{h}^{n}\right|\left|\boldsymbol{w}_{h}^{n+1}\right|\left\|A_{h}^{-1}\left(\boldsymbol{u}_{h}^{m+r}-\boldsymbol{u}_{h}^{m}\right)\right\|_{L^{\infty}(\Omega)} \\
& \leq C k^{2} \sum_{m=0}^{N-r} \sum_{n=m}^{m-1+r}\left|\boldsymbol{w}_{h}^{n+1}\right|\left\|A_{h}^{-1}\left(\boldsymbol{u}_{h}^{m+r}-\boldsymbol{u}_{h}^{m}\right)\right\|_{L^{\infty}(\Omega)} .
\end{aligned}
$$

Sobolev's inequality shows that

$\left\|A_{h}^{-1}\left(\boldsymbol{u}_{h}^{m+r}-\boldsymbol{u}_{h}^{m}\right)\right\|_{L^{\infty}(\Omega)} \leq C\left\|A^{-1}\left(\boldsymbol{u}_{h}^{m+r}-\boldsymbol{u}_{h}^{m}\right)\right\|_{W^{1, r}(\Omega)}$

with $r>d, d$ being the dimension of $\Omega$. The following bound

$$
\begin{aligned}
& \left\|A_{h}^{-1}\left(\boldsymbol{u}_{h}^{m+r}-\boldsymbol{u}_{h}^{m}\right)\right\|_{W^{1, r}(\Omega)} \\
& \quad \leq C\left\|A^{-1}\left(\boldsymbol{u}_{h}^{m+r}-\boldsymbol{u}_{h}^{m}\right)\right\|_{W^{1, r}(\Omega)},
\end{aligned}
$$

is proved in [25], under the regularity (32) with $r>d$ for $A^{-1}$ the Stokes operator (31). Thus, applying Sobolev's inequality, $H^{2}(\Omega) \hookrightarrow W^{1, r}(\Omega)$, with $r \leq 6$, gives

$$
\begin{aligned}
\left\|A^{-1}\left(\boldsymbol{u}_{h}^{m+r}-\boldsymbol{u}_{h}^{m}\right)\right\|_{L^{\infty}(\Omega)} & \leq C\left\|A^{-1}\left(\boldsymbol{u}_{h}^{m+r}-\boldsymbol{u}_{h}^{m}\right)\right\|_{H^{2}(\Omega)} \\
& \leq C\left\|\boldsymbol{u}_{h}^{m+r}-\boldsymbol{u}_{h}^{m}\right\|_{L^{2}(\Omega)}
\end{aligned}
$$


where we have used the regularity result (32) for $r=2$. Therefore,

$$
\begin{aligned}
J_{3} \leq & C k^{2} \sum_{m=0}^{N-r} \sum_{n=m}^{m-1+r}\left\|\boldsymbol{w}_{h}^{n+1}\right\|_{L^{2}(\Omega)}\left\|\boldsymbol{u}_{h}^{m+r}-\boldsymbol{u}_{h}^{m}\right\|_{L^{2}(\Omega)} \\
\leq & k \sum_{m=0}^{N-r}\left\|\boldsymbol{u}_{h}^{m+r}-\boldsymbol{u}_{h}^{m}\right\|_{L^{2}(\Omega)} \\
& \times\left(k \sum_{n=m}^{m-1+r}\left\|\boldsymbol{w}_{h}^{n+1}\right\|_{L^{2}(\Omega)}^{2}\right)^{1 / 2}(r k)^{1 / 2} \leq C(r k)^{1 / 2} .
\end{aligned}
$$

Finally, we conclude that

$k \sum_{m=0}^{N-r}\left\|\nabla A_{h}^{-1}\left(\boldsymbol{u}_{h}^{m+r}-\boldsymbol{u}_{h}^{m}\right)\right\|_{L^{2}(\Omega)}^{2} \leq C(r k)^{1 / 2}$,

which is equivalent to

$$
\begin{aligned}
& \int_{0}^{T-\delta}\left\|\boldsymbol{u}_{h, k, \varepsilon}(t+\delta)-\boldsymbol{u}_{h, k, \varepsilon}(t)\right\|_{X_{h}^{\prime}}^{2} d t \leq C \delta^{1 / 2} \\
& \quad \forall \delta: 0<\delta<T
\end{aligned}
$$

due to the fact that $\left\|\nabla A_{h}^{-1} \boldsymbol{u}_{h}\right\|_{L^{2}(\Omega)}$ and $\left\|\boldsymbol{u}_{h}\right\|_{X_{h}^{\prime}}$ are equivalent norms.

Note that the time fractional time estimate for the discrete velocity is bounded in the $\boldsymbol{V}_{h}^{\prime}$ norm, which depends on the space parameter $h$; therefore we cannot apply the compactness results given by Simon in [54]. Then the idea will be to encounter a fractional time norm being independent of the mesh parameters. Consider the space $\boldsymbol{V}=\{\boldsymbol{u} \in$ $\left.\boldsymbol{H}_{0}^{1}(\Omega): \nabla \cdot \boldsymbol{u}=0\right\}$ and the orthogonal projection $R_{h}$ : $\boldsymbol{V}_{h} \rightarrow \boldsymbol{V}$ such that $\left(\nabla\left(R_{h} \boldsymbol{u}_{h}-\boldsymbol{u}_{h}\right), \nabla \boldsymbol{v}\right)=0, \forall \boldsymbol{v} \in \boldsymbol{V}$. One knows from [29] that $\left\|R_{h} \boldsymbol{u}_{h}\right\|_{\boldsymbol{V}^{\prime}} \leq C\left(h\left|\nabla \cdot \boldsymbol{u}_{h}\right|+\left\|\boldsymbol{u}_{h}\right\|_{\boldsymbol{V}_{h}^{\prime}}\right)$ and

$$
\int_{0}^{T-\delta}\left\|R_{h} \boldsymbol{u}_{h, k, \varepsilon}(t+\delta)-R_{h} \boldsymbol{u}_{h, k, \varepsilon}(t)\right\|_{V^{\prime}}^{2} d t \leq C \delta^{1 / 2}+C h .
$$

Finally, the compactness of $\left\{R_{h} \boldsymbol{u}_{h, k, \varepsilon}^{l}\right\}$ in $L^{2}\left(0, T ; L^{2}(\Omega)\right)$ follows by a perturbed compactness result due to Azérad and Guillén-González in [3]. To conclude with the strong convergence, one uses the external approximation from $\boldsymbol{X}_{h}$ to $\boldsymbol{V}$, in order to prove that the sequence $\left\{\boldsymbol{u}_{h, k, \varepsilon}^{l}\right\}$ is compact in $L^{2}\left(0, T ; \boldsymbol{L}^{2}(\Omega)\right)$. To complete with the convergence we must pass to the limit. In [29], the authors needed to impose the additional hypothesis $(\mathrm{C})$ in the process. Although, scheme (67) is unconditionally stable, it may need such a condition.

An interesting issue is to prove compactness under the minimum assumptions over the boundary of $\Omega$. At the continuous level, one would need the Neumann problem to have the regularity (34) for $r=2$ when a semi-Galerkin method is used, while the discrete compactness result needs the Stokes problem to have the regularity (32) for $r>d$, with $d$ the space dimension. For the Ginzburg-Landau problem (11), it was attained in [23] for fixed $\varepsilon$.

\subsubsection{A Second-Order Scheme for the Ginzburg-Landau Problem}

We next discuss the work of Lin, Liu and Zhang in [41]. They presented the first numerical work preserving an exact discrete energy law for (38), i.e. without introducing numerical diffusion provided by the time-stepping schemes. It relies on a modified Crank-Nicolson or midpoint scheme: given $\left(\boldsymbol{u}_{h}^{n}, \boldsymbol{d}_{h}^{n}\right) \in\left(\boldsymbol{V}_{h}, \boldsymbol{D}_{h}\right)$, find $\left(\boldsymbol{u}_{h}^{n+1}, \boldsymbol{w}_{h}^{n+1}\right) \in \boldsymbol{V}_{h} \times \boldsymbol{W}_{h}$ and $\left(p_{h}^{n+1}, \boldsymbol{d}_{h}^{n+1}\right) \in P_{h} \times \boldsymbol{D}_{h}$ solving the finite element nonlinear system:

$$
\left\{\begin{array}{l}
\left(\delta_{t} \boldsymbol{u}_{h}^{n+1}, \overline{\boldsymbol{u}}_{h}\right)+\widetilde{c}\left(\boldsymbol{u}_{h}^{n+1 / 2}, \boldsymbol{u}_{h}^{n+1 / 2}, \overline{\boldsymbol{u}}_{h}\right) \\
\quad+v a\left(\boldsymbol{u}_{h}^{n+1 / 2}, \overline{\boldsymbol{u}}_{h}\right)-b_{u}\left(p_{h}^{n+1 / 2}, \overline{\boldsymbol{u}}_{h}\right) \\
\quad-\frac{\lambda}{\gamma} c\left(\overline{\boldsymbol{u}}_{h}, \boldsymbol{d}_{h}^{n+1 / 2}, \delta_{t} \boldsymbol{d}_{h}^{n+1}+\left(\boldsymbol{u}_{h}^{n+1 / 2} \cdot \nabla\right) \boldsymbol{d}_{h}^{n+1 / 2}\right)=0 \\
b_{u}\left(\bar{p}_{h}, \boldsymbol{u}_{h}^{n+1 / 2}\right)=0 \\
\left(\delta_{t} \boldsymbol{d}_{h}^{n+1}, \overline{\boldsymbol{d}}_{h}\right)+c\left(\boldsymbol{u}_{h}^{n+1 / 2}, \boldsymbol{d}_{h}^{n+1 / 2}, \overline{\boldsymbol{d}}_{h}\right) \\
\quad+\gamma a\left(\boldsymbol{d}_{h}^{n+1 / 2}, \overline{\boldsymbol{d}}_{h}\right)+\gamma\left(\boldsymbol{f}_{\varepsilon}\left(\boldsymbol{d}_{h}^{n+1}, \boldsymbol{d}_{h}^{n}\right), \overline{\boldsymbol{d}}_{h}\right)=0
\end{array}\right.
$$

where

$\boldsymbol{f}_{\varepsilon}\left(\boldsymbol{d}_{h}^{n}, \boldsymbol{d}_{h}^{n+1}\right)=\frac{1}{\varepsilon^{2}} \frac{\left(\left|\boldsymbol{d}_{h}^{n+1}\right|^{2}-1\right)+\left(\left|\boldsymbol{d}_{h}^{n}\right|^{2}-1\right)}{2} \boldsymbol{d}_{h}^{n+1 / 2}$.

Clearly, this approximation of $\boldsymbol{f}_{\varepsilon}(\boldsymbol{d})$ has second order of accuracy in time. The energy law given by (73) takes the form

$$
\begin{gathered}
\frac{1}{2}\left\|\boldsymbol{u}_{h}^{n+1}\right\|_{L^{2}(\Omega)}^{2}+\frac{\lambda}{2}\left\|\nabla \boldsymbol{d}_{h}^{n+1}\right\|_{L^{2}(\Omega)}^{2}+\lambda \int_{\Omega} F_{\varepsilon}\left(\boldsymbol{d}_{h}^{n+1}\right) \\
+v k \sum_{m=1}^{n+1}\left\|\nabla \boldsymbol{u}_{h}^{m+1 / 2}\right\|_{L^{2}(\Omega)}^{2} \\
+\frac{\lambda}{\gamma} k \sum_{m=1}^{n+1}\left\|\delta_{t} \boldsymbol{d}_{h}^{m+1}+\left(\boldsymbol{u}_{h}^{m+\frac{1}{2}} \cdot \nabla\right) \boldsymbol{d}_{h}^{m+\frac{1}{2}}\right\|_{L^{2}(\Omega)}^{2} \\
=\frac{1}{2}\left\|\boldsymbol{u}_{h}^{0}\right\|_{L^{2}(\Omega)}^{2}+\frac{\lambda}{2}\left\|\nabla \boldsymbol{d}_{h}^{0}\right\|_{L^{2}(\Omega)}^{2}+\int_{\Omega} F_{\varepsilon}\left(\boldsymbol{d}_{h}^{0}\right)
\end{gathered}
$$

for all $n$.

The fact that no auxiliary variable is introduced is a clear benefit of this approach. In contract, since scheme (73) is nonlinear, one needs to perform nonlinear iterations. The authors proposed a Picard type linearization together with 
a block Gauss-Seidel solver and a fully explicit treatment of the nonlinear terms, in order to have a time-independent time matrix and use an exact LU or Cholesky decomposition only once. Let us define $\phi_{s-1 / 2}=\frac{\phi_{h}^{n}+\phi_{h}^{s-1}}{2}$ and $\phi_{S+1 / 2}=$ $\frac{\phi_{h}^{s}+\phi_{h}^{n}}{2}$. Then, given $\left(\boldsymbol{u}_{h}^{n}, \boldsymbol{d}_{h}^{n}\right) \in\left(\boldsymbol{V}_{h}, \boldsymbol{D}_{h}\right)$, the methods reads as:

1. Known $\boldsymbol{u}_{s-1}$, find $\left(\boldsymbol{u}_{s}, p_{s}\right) \in \boldsymbol{V}_{h} \times P_{h}$ such that

$$
\begin{gathered}
\left(\frac{\boldsymbol{u}_{h}^{s}-\boldsymbol{u}_{h}^{n}}{k}, \overline{\boldsymbol{u}}_{h}\right)+\widetilde{c}\left(\boldsymbol{u}_{s-1 / 2}, \boldsymbol{u}_{s-1 / 2}, \overline{\boldsymbol{u}}_{h}\right) \\
+\nu a\left(\boldsymbol{u}_{s+1 / 2}, \overline{\boldsymbol{u}}_{h}\right)-b_{u}\left(p_{s+1 / 2}, \overline{\boldsymbol{u}}_{h}\right) \\
\quad-\lambda c\left(\overline{\boldsymbol{u}}_{h}, \boldsymbol{d}_{s-1 / 2}, \frac{\boldsymbol{d}_{h}^{s-1}-\boldsymbol{d}_{h}^{n}}{k}\right. \\
\left.\quad+\left(\boldsymbol{u}_{s-1 / 2} \cdot \nabla\right) \boldsymbol{d}_{s-1 / 2}\right)=0, \\
b_{u}\left(\bar{p}_{h}, \boldsymbol{u}_{s+1 / 2}\right)=0 .
\end{gathered}
$$

2. Next, find $\boldsymbol{d}_{s} \in \boldsymbol{D}_{h}$ such that

$$
\begin{aligned}
& \left(\frac{\boldsymbol{d}_{h}^{s}-\boldsymbol{d}_{h}^{n}}{k}, \overline{\boldsymbol{d}}_{h}\right)+c\left(\boldsymbol{u}_{s-1 / 2}, \boldsymbol{d}_{s-1 / 2}, \overline{\boldsymbol{d}}_{h}\right) \\
& \quad+a\left(\boldsymbol{d}_{s+1 / 2}, \overline{\boldsymbol{d}}_{h}\right)+\left(\boldsymbol{f}_{\varepsilon}\left(\boldsymbol{d}_{h}^{n}, \boldsymbol{d}_{h}^{s-1}\right), \overline{\boldsymbol{d}}_{h}\right)=0 .
\end{aligned}
$$

The use of direct solvers is restricted to very small problems. For real applications, iterative methods are the only choice, due to CPU and memory limitations. In those cases, this way to deal with coupling and nonlinearities is very rudimentary. More modern approaches to linearization and linear solvers, e.g. Newton-Krylov-type solvers, surely provide better results.

There is no numerical analysis for scheme (73). The convergence in the sense of error estimates or compactness is a interesting question that is open.

\section{On the Approximation of the Ericksen-Leslie Problem (3)}

This section is devoted to finite element schemes for the Ericksen-Leslie equations (3) that provide a discrete energy law. To obtain the energy law (7) we need the sphere constraint $|\boldsymbol{d}|=1$ to be fulfilled almost everywhere in $Q$ which is difficult to achieve at the discrete level due to the own nature of Lagrange finite elements. Therefore, constructing stable numerical approximations directly for (3) results a more difficult task than for the Ginzburg-Landau problem (11).

\subsection{A Direct Approximation}

The next scheme we will present is due to Becker, Feng, and Prohl in [8]. The numerical approximations are based on the ideas given in [7] for the unsteady harmonic map equation which utilizes the Galerkin method with Lagrange finite elements of order 1 . The vector identity $\boldsymbol{a} \times(\boldsymbol{b} \times \boldsymbol{c})=$ $(\boldsymbol{a} \cdot \boldsymbol{c}) \boldsymbol{b}-(\boldsymbol{a} \cdot \boldsymbol{b}) \boldsymbol{c}$ for all $\boldsymbol{a}, \boldsymbol{b}, \boldsymbol{c} \in \mathbb{R}^{3}$ and $|\boldsymbol{d}|=1$ lead to the identity

$\boldsymbol{d} \times(\boldsymbol{d} \times \Delta \boldsymbol{d})=-|\nabla \boldsymbol{d}|^{2} \boldsymbol{d}-\Delta \boldsymbol{d}$.

Therefore, problem (3) becomes

$\begin{cases}\partial_{t} \boldsymbol{u}+\boldsymbol{u} \cdot \nabla \boldsymbol{u}-v \Delta \boldsymbol{u} & \\ \quad+\nabla p+\lambda \nabla \cdot\left((\nabla \boldsymbol{d})^{t} \nabla \boldsymbol{d}\right)=\mathbf{0} & \text { in } Q, \\ \nabla \cdot \boldsymbol{u}=0 & \text { in } Q, \\ \partial_{t} \boldsymbol{d}+\boldsymbol{u} \cdot \nabla \boldsymbol{d}+\gamma \boldsymbol{d} \times(\boldsymbol{d} \times \Delta \boldsymbol{d})=\mathbf{0} & \text { in } Q, \\ |\boldsymbol{d}|=1 & \text { in } Q .\end{cases}$

From (74) 3 , it is not hard to prove that $|\boldsymbol{d}|=1$ holds almost everywhere in $Q$. Therefore, the set of (3) $3_{3-4}$ is equivalent to $(74)_{3}$. Based on that, we consider that system (74) is the best differential reformulation of (3). But it has its limitations when designing numerical schemes.

Let us first state the variational formulation by using the elastic stress expression (6). Find $(\boldsymbol{u}(t), p(t), \boldsymbol{d}(t)) \in$ $\boldsymbol{H}_{0}^{1}(\Omega) \times L_{0}^{2}(\Omega) \times \boldsymbol{H}^{2}(\Omega)$ such that

$$
\left\{\begin{array}{c}
\left(\partial_{t} \boldsymbol{u}, \overline{\boldsymbol{u}}\right)+c(\boldsymbol{u}, \boldsymbol{u}, \overline{\boldsymbol{u}})+v a(\boldsymbol{u}, \overline{\boldsymbol{u}}) \\
\quad-b_{u}(p, \overline{\boldsymbol{u}})+\lambda c(\overline{\boldsymbol{u}}, \boldsymbol{d}, \boldsymbol{w})=0, \\
b_{u}(\bar{p}, \boldsymbol{u})=0, \\
\left(\partial_{t} \boldsymbol{d}, \overline{\boldsymbol{d}}\right)+c(\boldsymbol{u}, \boldsymbol{d}, \overline{\boldsymbol{d}}) \\
\quad+\gamma(\boldsymbol{d} \times(\boldsymbol{d} \times \Delta \boldsymbol{d}), \overline{\boldsymbol{d}})=0,
\end{array}\right.
$$

for all $(\overline{\boldsymbol{u}}, \bar{p}, \overline{\boldsymbol{d}}) \in \boldsymbol{H}_{0}^{1}(\Omega) \times L_{0}^{2}(\Omega) \times \boldsymbol{L}^{2}(\Omega)$.

In order to reach the energy law that stems form (74) we multiply (74) 1 by $\boldsymbol{u}$ and (74) 3 by $-\Delta \boldsymbol{d}$ and integrate over $\Omega$. Then the identity $(\boldsymbol{a} \times \boldsymbol{b}) \cdot \boldsymbol{a}=-(\boldsymbol{a} \times \boldsymbol{c}) \cdot \boldsymbol{b}$ leads to

$$
\begin{aligned}
& \frac{d}{d t}\left(\frac{1}{2}\|\boldsymbol{u}\|_{L^{2}(\Omega)}^{2}+\frac{\lambda}{2}\|\nabla \boldsymbol{d}\|_{L^{2}(\Omega)}^{2}\right) \\
& \quad+v\|\nabla \boldsymbol{u}\|_{L^{2}(\Omega)}^{2}+\lambda \gamma\|\Delta \boldsymbol{d} \times \boldsymbol{d}\|_{L^{2}(\Omega)}^{2}=0 .
\end{aligned}
$$

The discrete problem for (75) proposed in [8] uses the finite element spaces $V_{h}=\left(\left(\mathcal{P}_{h}^{1}\right)^{d} \oplus\left(\mathcal{B}_{h}\right)^{d}\right) \cap \boldsymbol{H}_{0}^{1}(\Omega), P_{h}=$ $\mathcal{P}_{h}^{1} \cap L_{0}^{2}(\Omega)$, and $\boldsymbol{D}_{h}=\left(\mathcal{P}_{h}^{1}\right)^{d}$ for the discrete velocity, pressure and director, respectively. Moreover, the scheme uses an implicit time integration for the linear terms, semiimplicit time integration for the convective terms, a midpoint time integration for the trilinear term in $(75)_{3}$ and semimidpoint rule for the elastic stress tensor in $(75)_{1}$. Therefore, if we are given $\left(\boldsymbol{u}_{h}^{n}, \boldsymbol{d}_{h}^{n}\right) \in\left(\boldsymbol{V}_{h}, \boldsymbol{D}_{h}\right)$, we want to find a 
discrete solution $\left(\boldsymbol{u}_{h}^{n+1}, p_{h}^{n+1}, \boldsymbol{d}_{h}^{n+1}\right) \in \boldsymbol{V}_{h} \times P_{h} \times \boldsymbol{D}_{h}$ solving the finite nonlinear system:

$$
\left\{\begin{array}{l}
\left(\delta_{t} \boldsymbol{u}_{h}^{n+1}, \overline{\boldsymbol{u}}_{h}\right)+\widetilde{c}\left(\boldsymbol{u}_{h}^{n}, \boldsymbol{u}_{h}^{n+1}, \overline{\boldsymbol{u}}_{h}\right) \\
\quad+v a\left(\boldsymbol{u}_{h}^{n+1}, \overline{\boldsymbol{u}}_{h}\right)-b_{u}\left(p_{h}^{n+1}, \overline{\boldsymbol{u}}_{h}\right) \\
\quad+\lambda c\left(\overline{\boldsymbol{u}}_{h}, \boldsymbol{d}_{h}^{n+1}, \Delta_{h} \boldsymbol{d}_{h}^{n+1 / 2}\right)=0, \\
b_{u}\left(\bar{p}_{h}, \boldsymbol{u}_{h}^{n}\right)=0, \\
\left(\delta_{t} \boldsymbol{d}_{h}^{n+1}, \overline{\boldsymbol{d}}_{h}\right)+c\left(\boldsymbol{u}_{h}^{n+1}, \boldsymbol{d}_{h}^{n}, \overline{\boldsymbol{d}}_{h}\right) \\
\quad+\gamma\left(\boldsymbol{d}_{h}^{n+1 / 2} \times\left(\boldsymbol{d}_{h}^{n+1 / 2} \times \Delta_{h} \boldsymbol{d}_{h}^{n+1 / 2}\right), \overline{\boldsymbol{d}}_{h}\right)=0
\end{array}\right.
$$

for all $\left(\overline{\boldsymbol{u}}_{h}, \bar{p}_{h}, \overline{\boldsymbol{d}}_{h}\right) \in \boldsymbol{V}_{h} \times P_{h} \times \boldsymbol{D}_{h}$, where $\Delta_{h}: \boldsymbol{H}^{1}(\Omega) \rightarrow$ $\boldsymbol{D}_{h}$ is the discrete Laplace operator defined by

$-\left(\Delta_{h} \boldsymbol{d}, \overline{\boldsymbol{d}}_{h}\right)=\left(\nabla \boldsymbol{d}, \nabla \overline{\boldsymbol{d}}_{h}\right) \quad$ for all $\overline{\boldsymbol{d}}_{h} \in \boldsymbol{D}_{h}$.

It is not hard to prove the discrete analog to (76) from (77) without any extra condition for the parameters:

$$
\begin{aligned}
& \frac{1}{2}\left\|\boldsymbol{u}_{h}^{n+1}\right\|_{L^{2}(\Omega)}^{2}+\frac{\lambda}{2}\left\|\nabla \boldsymbol{d}_{h}^{n+1}\right\|_{L^{2}(\Omega)}^{2} \\
& \quad+v k \sum_{m=1}^{n+1}\left\|\nabla \boldsymbol{u}_{h}^{m+1 / 2}\right\|_{L^{2}(\Omega)}^{2} \\
& \quad+\lambda \gamma k \sum_{m=1}^{n+1}\left\|\boldsymbol{d}_{h}^{m+1 / 2} \times \Delta_{h} \boldsymbol{d}_{h}^{m+1 / 2}\right\|_{L^{2}(\Omega)}^{2} \\
& \leq \frac{1}{2}\left\|\boldsymbol{u}_{h}^{0}\right\|_{L^{2}(\Omega)}^{2}+\frac{\lambda}{2}\left\|\nabla \boldsymbol{d}_{h}^{0}\right\|_{L^{2}(\Omega)}^{2} .
\end{aligned}
$$

Since the Dirichlet seminorm $\left\|\nabla \boldsymbol{d}_{h}^{n+1}\right\|_{L^{2}(\Omega)}$ is not equivalent to the norm $\left\|\boldsymbol{d}_{h}^{n+1}\right\|_{H^{1}(\Omega)}$ in this case, the existence of $\left(\boldsymbol{u}_{h}^{n+1}, \boldsymbol{d}_{h}^{n+1}\right)$ to (77) is established for the $L^{2}(\Omega)$ norm under the restriction $k=\mathcal{O}\left(h^{2+d / 2}\right)$ by means of a fixed point argument; we refer the reader to Corollary 1.1 on p. 279 of [26] or Lemma 1.4 on page 164 of [55]. Of course, this problem does not appear for time-independent nonhomogeneous Dirichlet boundary conditions. Therefore, scheme (77) seems to be unconditionally solvable.

The following task is to know how the sphere constraint holds for the director field in the limit for the scheme (77). In [7] a sharp proof is given for two-dimensional domains. If the initial director field satisfied $\left|\boldsymbol{d}_{h}^{0}(\boldsymbol{z})\right|=1$ for all nodes $z \in \mathcal{N}_{h}$ and under the relation $k=\mathcal{O}\left(h^{3}\right)$, one gets

$$
\left\|\left|\boldsymbol{d}_{h}^{n+1}\right|^{2}-1\right\|_{L^{2}(\Omega)} \leq\left\|\left|\boldsymbol{d}_{h}^{0}\right|^{2}-1\right\|_{L^{2}(\Omega)}+g(h),
$$

where $g(h) \rightarrow 0$ when $h \rightarrow 0$. Such an estimate can be seen in term of the potential function associated to the Ginzburg-Landau penalty function as $F_{\varepsilon}\left(\boldsymbol{d}_{h}^{n+1}\right) \leq C$ for $\varepsilon=\left\|\left|\boldsymbol{d}_{h}^{0}\right|^{2}-1\right\|_{L^{2}(\Omega)}^{2}+g^{2}(h)$. While the first term on the right-hand side of (78) is of order $h$, the second term is of order $h^{\alpha}$ for some $\alpha<1$. Along the proof the authors used some sort of Poincaré inequality to be able to obtain $\left\|\boldsymbol{d}_{h}^{n}\right\|_{H^{1}(\Omega)} \leq C\left\|\nabla \boldsymbol{d}_{h}^{n}\right\|_{L^{2}(\Omega)}$, but it is not clear from the Neumann boundary condition imposed on the director vector. However, for time-independent Dirichlet boundary conditions, that Poincaré inequality is straightforwardly obtained by a lifting of the boundary condition. Note that the restriction $k=\mathcal{O}\left(h^{3}\right)$ is equivalent to hypothesis (S) for scheme (47) in two-dimensional domains.

The following estimate for the discrete time derivative of the discrete director field is needed to obtain (78):

$k \sum_{n=0}^{N-1}\left\|\delta_{t} \boldsymbol{d}_{h}^{n+1}\right\|_{L^{p}(\Omega)}^{2} \leq C$

for $p<2$ and $p=3 / 2$ in two- and three-dimensional domains, respectively. This estimate is attained by a duality argument. But again a Poincaré inequality is needed.

The convergence of scheme (77) is as an open problem even towards measure-valued solutions [38, 39].

\subsection{A Saddle-Point Formulation}

When expressed in the appropriate mathematical setting, one realizes that all the above numerical method described are connected. Lagrange multiplier methods allow one to introduce a unified formulation leading to a numerical algorithm for the Ginzburg-Landau equations (11) and the Ericksen-Leslie equations (3). The following variational form of (27) was proposed in [5]. Consider the term $\lambda \nabla$. $\left((\nabla \boldsymbol{d})^{t} \nabla \boldsymbol{d}\right)$ written in a similar way to (38) done in [40, 41] which saves to compute an extra variable as for schemes (43), (47), (67) and (69). Then the problem consists of finding $(\boldsymbol{u}(t), p(t), \boldsymbol{d}(t), q(t)) \in \boldsymbol{H}_{0}^{1}(\Omega) \times L_{0}^{2}(\Omega) \times \boldsymbol{W}^{1,3}(\Omega) \cap$ $L^{\infty}(\Omega) \times Q(\Omega)$ such that

$$
\left\{\begin{array}{l}
\left(\partial_{t} \boldsymbol{u}, \overline{\boldsymbol{u}}\right)+c(\boldsymbol{u}, \boldsymbol{u}, \overline{\boldsymbol{u}})+v a(\boldsymbol{u}, \overline{\boldsymbol{u}}) \\
\quad-b_{u}(p, \overline{\boldsymbol{u}})+\frac{\lambda}{\gamma} c\left(\overline{\boldsymbol{u}}, \boldsymbol{d}, \partial_{t} \boldsymbol{d}+(\boldsymbol{u} \cdot \nabla) \boldsymbol{d}\right)=0 \\
b_{u}(\bar{p}, \boldsymbol{u})=0, \\
\left(\partial_{t} \boldsymbol{d}, \overline{\boldsymbol{d}}\right)+c(\boldsymbol{u}, \boldsymbol{d}, \overline{\boldsymbol{d}})+\gamma a(\boldsymbol{d}, \overline{\boldsymbol{d}})+\gamma b_{d}(q, \boldsymbol{d}, \overline{\boldsymbol{d}})=0 \\
b_{d}(\bar{q}, \boldsymbol{d}, \boldsymbol{d})-\varepsilon^{2}(q, \bar{q})=\langle 1, \bar{q}\rangle
\end{array}\right.
$$

for all $(\boldsymbol{u}(t), p(t), \boldsymbol{d}(t), q(t)) \in \boldsymbol{H}_{0}^{1}(\Omega) \times L_{0}^{2}(\Omega) \times \boldsymbol{H}^{1}(\Omega) \times$ $Q(\Omega)^{\prime}$. The space $Q(\Omega)$ is $L^{2}(\Omega)$ when $\varepsilon>0$ or $Q(\Omega)$ is the dual space of $H^{1}(\Omega)$ denoted by $\left(H^{1}(\Omega)\right)^{\prime}$ when $\varepsilon=0$. It is easy to see that the weak formulation (79) is well-defined on the previous spaces. Note that for twodimensional domains $\boldsymbol{d}$ may only belong to $\boldsymbol{W}^{1,3}(\Omega)$ due to Sobolev's inequality $W^{1,3}(\Omega) \hookrightarrow L^{\infty}(\Omega)$. The idea to consider the Lagrange multiplier to belong to $\left(H^{1}(\Omega)\right)^{\prime}$ stemmed from the inf-sup condition (24). 
Another possible formulation studied in [5] consists in replacing the weak formulation

$b_{d}(\bar{q}, \boldsymbol{d}, \boldsymbol{d})-\varepsilon^{-2}(q, \bar{q})=\langle 1, \bar{q}\rangle$

of the sphere constraint by its derivative in time

$2 b_{d}\left(\bar{q}, \boldsymbol{d}, \partial_{t} \boldsymbol{d}\right)-\varepsilon^{2}\left(\partial_{t} q, \bar{q}\right)=0 \quad$ for all $\bar{q} \in Q(\Omega)$.

\section{An Implicit Algorithm}

A first attempt to discretize (79) is an implicit Euler scheme. So, let $\boldsymbol{d}_{h}^{n} \in \boldsymbol{D}_{h}$ and $\boldsymbol{u}_{h}^{n} \in \boldsymbol{V}_{h}$ be given. Then find $\left(\boldsymbol{u}_{h}^{n+1}, p_{h}^{n+1}, \boldsymbol{d}_{h}^{n+1}, q_{h}^{n+1},\right) \in \boldsymbol{V}_{h} \times P_{h} \times \boldsymbol{D}_{h} \times Q_{h}$ such that

$$
\left\{\begin{array}{l}
\left(\delta_{t} \boldsymbol{u}_{h}^{n+1}, \overline{\boldsymbol{u}}_{h}\right)+\widetilde{c}\left(\boldsymbol{u}_{h}^{n+1}, \boldsymbol{u}_{h}^{n+1}, \overline{\boldsymbol{u}}_{h}\right) \\
\quad+v a\left(\boldsymbol{u}_{h}^{n+1}, \overline{\boldsymbol{u}}_{h}\right)+b_{u}\left(p_{h}^{n+1}, \overline{\boldsymbol{u}}_{h}\right) \\
\quad+\frac{\lambda}{\gamma} c\left(\overline{\boldsymbol{u}}_{h}, \boldsymbol{d}_{h}^{n+1}, \delta_{t} \boldsymbol{d}_{h}^{n+1}+\left(\boldsymbol{u}_{h}^{n+1} \cdot \nabla\right) \boldsymbol{d}_{h}^{n+1}\right)=0 \\
b_{u}\left(\bar{q}_{h}, \boldsymbol{u}_{h}^{n+1}\right)=0 \\
\left(\delta_{t} \boldsymbol{d}_{h}^{n+1}, \overline{\boldsymbol{d}}_{h}\right)+\gamma a\left(\boldsymbol{d}_{h}^{n+1}, \overline{\boldsymbol{d}}_{h}\right) \\
\quad+c\left(\boldsymbol{u}_{h}^{n+1}, \boldsymbol{d}_{h}^{n+1}, \overline{\boldsymbol{d}}_{h}\right)+\gamma b_{d}\left(q_{h}^{n+1}, \boldsymbol{d}_{h}^{n+1}, \overline{\boldsymbol{d}}_{h}\right)=0 \\
2 b_{d}\left(\bar{q}_{h}, \boldsymbol{d}_{h}^{n+1}, \delta_{t} \boldsymbol{d}_{h}^{n+1}\right)-\varepsilon^{2}\left(\delta_{t} q_{h}^{n+1}, \bar{q}_{h}\right)=0
\end{array}\right.
$$

for all

$\left(\overline{\boldsymbol{u}}_{h}, \bar{p}_{h}, \overline{\boldsymbol{d}}_{h}, \bar{q}_{h}\right) \in \boldsymbol{V}_{h} \times P_{h} \times \boldsymbol{D}_{h} \times Q_{h}$

where the finite element spaces are $V_{h}=\left(\left(\mathcal{P}_{h}^{1}\right)^{d} \oplus\left(\mathcal{B}_{h}\right)^{d}\right) \cap$ $\boldsymbol{H}_{0}^{1}(\Omega), P_{h}=\mathcal{P}_{h}^{1} \cap L_{0}^{2}\left(\Omega, \boldsymbol{D}_{h}=\left(\mathcal{P}_{h}^{1}\right)^{d}\right.$, and $Q_{h}=\mathcal{P}_{h}^{1}$. Observe that $(81)_{4}$ is the discrete version of the alternative equation (80) since if we considered

$b_{d}\left(\bar{q}_{h}, \boldsymbol{d}_{h}^{n+1}, \boldsymbol{d}_{h}^{n+1}\right)-\varepsilon^{-2}\left(q_{h}^{n+1}, \bar{q}_{h}\right)=\left\langle 1, \bar{q}_{h}\right\rangle$

an energy estimate would not be clear.

The following energy inequality holds for scheme (81):

$$
\begin{aligned}
& \frac{1}{2}\left\|\boldsymbol{u}_{h}^{n+1}\right\|_{L^{2}(\Omega)}^{2}+\frac{\lambda}{2}\left\|\nabla \boldsymbol{d}_{h}^{n+1}\right\|_{L^{2}(\Omega)}^{2}+\frac{\lambda \epsilon^{2}}{4}\left\|q_{h}^{n+1}\right\|_{L^{2}(\Omega)}^{2} \\
& +\nu k \sum_{m=0}^{n}\left\|\nabla \boldsymbol{u}_{h}^{m+1}\right\|_{L^{2}(\Omega)}^{2} \\
& \quad+\frac{\lambda}{\gamma} k \sum_{m=0}^{n}\left\|\delta_{t} \boldsymbol{d}_{h}^{m+1}+\left(\boldsymbol{u}_{h}^{m+1} \cdot \nabla\right) \boldsymbol{d}_{h}^{m+1}\right\|_{L^{2}(\Omega)}^{2} \\
& \leq \frac{1}{2}\left\|\boldsymbol{u}_{h}^{0}\right\|_{L^{2}(\Omega)}^{2}+\frac{\lambda}{2}\left\|\nabla \boldsymbol{d}_{h}^{0}\right\|_{L^{2}(\Omega)}^{2} \\
& +\frac{\lambda \varepsilon^{2}}{4}\left\|q_{h}^{0}\right\|_{L^{2}(\Omega)}^{2} \quad \text { for all } n .
\end{aligned}
$$

A Crank-Nicolson Algorithm

If we want to get an unconditionally stable scheme preserving the energy law (7) for $\varepsilon=0$ or (12) for $\varepsilon>0$, we should consider a Crank-Nicolson time integration. Thus, given $\left(\boldsymbol{u}_{h}^{n}, \boldsymbol{d}_{h}^{n}\right) \in \boldsymbol{V}_{h} \times D_{h}$, we seek the discrete solution $\left(\boldsymbol{u}_{h}^{n+1}, p_{h}^{n+1}, \boldsymbol{d}_{h}^{n+1}, q_{h}^{n+1}\right) \in \boldsymbol{V}_{h} \times P_{h} \times \boldsymbol{D}_{h} \times Q_{h}$ such that

$$
\left\{\begin{array}{l}
\left(\delta_{t} \boldsymbol{u}_{h}^{n+1}, \overline{\boldsymbol{u}}_{h}\right)+\widetilde{c}\left(\boldsymbol{u}_{h}^{n+1 / 2}, \boldsymbol{u}_{h}^{n+1 / 2}, \overline{\boldsymbol{u}}_{h}\right)+v a\left(\boldsymbol{u}_{h}^{n+1 / 2}, \overline{\boldsymbol{u}}_{h}\right) \\
\quad+b_{u}\left(p_{h}^{n+1 / 2}, \overline{\boldsymbol{u}}_{h}\right)+\frac{\lambda}{\gamma} c\left(\overline{\boldsymbol{u}}_{h}, \boldsymbol{d}_{h}^{n+1 / 2}, \delta_{t} \boldsymbol{d}_{h}^{n+1}\right. \\
\left.\quad+\left(\boldsymbol{u}_{h}^{n+1 / 2} \cdot \nabla\right) \boldsymbol{d}_{h}^{n+1 / 2}\right)=0, \\
b_{u}\left(\bar{q}_{h}, \boldsymbol{u}_{h}^{n+1 / 2}\right)=0, \\
\quad\left(\delta_{t} \boldsymbol{d}_{h}^{n+1 / 2}, \overline{\boldsymbol{d}}_{h}\right)+\gamma a\left(\boldsymbol{d}_{h}^{n+1 / 2}, \overline{\boldsymbol{d}}_{h}\right) \\
\quad+c\left(\boldsymbol{u}_{h}^{n+1 / 2}, \boldsymbol{d}_{h}^{n+1 / 2}, \overline{\boldsymbol{d}}_{h}\right) \\
\quad+\gamma b_{d}\left(q_{h}^{n+1 / 2}, \boldsymbol{d}_{h}^{n+1 / 2}, \overline{\boldsymbol{d}}_{h}\right)=0, \\
b_{d}\left(\bar{q}_{h}, \boldsymbol{d}_{h}^{n+1}, \boldsymbol{d}_{h}^{n+1}\right)-\varepsilon^{2}\left(q_{h}^{n+1}, \bar{q}_{h}\right)=\left\langle 1, \bar{q}_{h}\right\rangle
\end{array}\right.
$$

for all

$\left(\overline{\boldsymbol{u}}_{h}, \bar{p}_{h}, \overline{\boldsymbol{d}}_{h}, \bar{q}_{h}\right) \in \boldsymbol{V}_{h} \times P_{h} \times \boldsymbol{D}_{h} \times Q_{h}$.

Note that the restriction over $\boldsymbol{d}_{h}$ has been discretized by using an implicit time integration.

The following a priori energy equality holds for scheme (83):

$$
\begin{aligned}
& \frac{1}{2}\left\|\boldsymbol{u}_{h}^{n+1}\right\|_{L^{2}(\Omega)}^{2}+\frac{\lambda}{2}\left\|\nabla \boldsymbol{d}_{h}^{n+1}\right\|_{L^{2}(\Omega)}^{2}+\frac{\lambda \varepsilon^{2}}{4}\left\|q_{h}^{n+1}\right\|_{L^{2}(\Omega)}^{2} \\
& +v k \sum_{m=0}^{n}\left\|\nabla \boldsymbol{u}_{h}^{m+\frac{1}{2}}\right\|_{L^{2}(\Omega)}^{2} \\
& \quad+k \sum_{m=0}^{n} \frac{\beta}{\gamma}\left\|\delta_{t} \boldsymbol{d}_{h}^{m+\frac{1}{2}}+\left(\boldsymbol{u}_{h}^{m+\frac{1}{2}} \cdot \nabla\right) \boldsymbol{d}_{h}^{m+\frac{1}{2}}\right\|_{L^{2}(\Omega)}^{2} \\
& =\frac{1}{2}\left\|\boldsymbol{u}_{h}^{0}\right\|_{L^{2}(\Omega)}^{2}+\frac{\lambda}{2}\left\|\nabla \boldsymbol{d}_{h}^{0}\right\|_{L^{2}(\Omega)}^{2} \\
& +\frac{\lambda \varepsilon^{2}}{4}\left\|q_{h}^{0}\right\|_{L^{2}(\Omega)}^{2} \quad \text { for all } n .
\end{aligned}
$$

Note that an equality is now obtained, that is, the energy law is exactly conserved, since scheme (83) introduces no numerical dissipation.

\section{A Semi-Implicit Algorithm}

We next show a linear algorithm developed in [5], which is implicit for the linear terms and semi-implicit for the nonlinear terms.

Given $\left(\boldsymbol{u}_{h}^{n}, \boldsymbol{d}_{h}^{n}\right) \in \boldsymbol{V}_{h} \times \boldsymbol{D}_{h}$, we want to find finite element functions $\left(\boldsymbol{u}_{h}^{n+1}, p_{h}^{n+1}, \boldsymbol{d}_{h}^{n+1}, q_{h}^{n+1}\right) \in \boldsymbol{V}_{h} \times P_{h} \times \boldsymbol{D}_{h} \times Q_{h}$ 
such that

$$
\left\{\begin{array}{l}
\left(\delta_{t} \boldsymbol{u}_{h}^{n+1}, \overline{\boldsymbol{u}}_{h}\right)+\widetilde{c}\left(\boldsymbol{u}_{h}^{n}, \boldsymbol{u}_{h}^{n+1}, \overline{\boldsymbol{u}}_{h}\right) \\
\quad+v a\left(\boldsymbol{u}_{h}^{n+1}, \overline{\boldsymbol{u}}_{h}\right) \\
\quad+b_{u}\left(p_{h}^{n+1}, \overline{\boldsymbol{u}}_{h}\right)+\frac{\lambda}{\gamma} c\left(\overline{\boldsymbol{u}}_{h}, \boldsymbol{d}_{h}^{n}, \delta_{t} \boldsymbol{d}_{h}^{n+1}\right. \\
\left.\quad+\left(\boldsymbol{u}_{h}^{n+1} \cdot \nabla\right) \boldsymbol{d}_{h}^{n}\right)=0 \\
b_{u}\left(\bar{q}_{h}, \boldsymbol{u}_{h}^{n+1}\right)=0, \\
\left(\delta_{t} \boldsymbol{d}_{h}^{n+1}, \overline{\boldsymbol{d}}_{h}\right)+\gamma a\left(\boldsymbol{d}_{h}^{n+1}, \overline{\boldsymbol{d}}_{h}\right) \\
\quad+c\left(\boldsymbol{u}_{h}^{n+1}, \boldsymbol{d}_{h}^{n}, \overline{\boldsymbol{d}}_{h}\right)+\gamma b_{d}\left(q_{h}^{n}, \boldsymbol{d}_{h}^{n+1}, \overline{\boldsymbol{d}}_{h}\right)=0 \\
2 b_{d}\left(\bar{q}_{h}, \boldsymbol{d}_{h}^{n}, \delta_{t} \boldsymbol{d}_{h}^{n+1}\right)-\varepsilon^{2}\left(\delta_{t} q_{h}^{n+1}, \bar{q}_{h}\right)=0
\end{array}\right.
$$

for all $\left(\overline{\boldsymbol{u}}_{h}, \bar{p}_{h}, \overline{\boldsymbol{d}}_{h}, \bar{q}_{h}\right) \in \boldsymbol{V}_{h} \times P_{h} \times \boldsymbol{D}_{h} \times Q_{h}$. The discrete energy inequality that provides scheme (84) is as follows:

$$
\begin{aligned}
& \frac{1}{2}\left\|\boldsymbol{u}_{h}^{n+1}\right\|_{L^{2}(\Omega)}^{2}+\frac{\lambda}{2}\left\|\nabla \boldsymbol{d}_{h}^{n+1}\right\|_{L^{2}(\Omega)}^{2}+\frac{\lambda \epsilon^{2}}{4}\left\|q_{h}^{n+1}\right\|_{L^{2}(\Omega)}^{2} \\
& +\nu k \sum_{m=0}^{n}\left\|\nabla \boldsymbol{u}_{h}^{m+1}\right\|_{L^{2}(\Omega)}^{2} \\
& \quad+\frac{\lambda}{\gamma} k \sum_{m=0}^{n}\left\|\delta_{t} \boldsymbol{d}_{h}^{m+1}+\left(\boldsymbol{u}_{h}^{m+1} \cdot \nabla\right) \boldsymbol{d}_{h}^{m}\right\|_{L^{2}(\Omega)}^{2} \\
& \leq \frac{1}{2}\left\|\boldsymbol{u}_{h}^{0}\right\|_{L^{2}(\Omega)}^{2}+\frac{\lambda}{2}\left\|\nabla \boldsymbol{d}_{h}^{0}\right\|_{L^{2}(\Omega)}^{2} \\
& +\frac{\lambda \varepsilon^{2}}{4}\left\|q_{h}^{0}\right\|_{L^{2}(\Omega)}^{2} \quad \text { for all } n .
\end{aligned}
$$

Schemes (81), (83) and (84) turn out to be unconditionally long-time stable with decreasing discrete energy. In particular, scheme (84) is the first linear scheme for both the Ginzburg-Landau problem and the Ericksen-Leslie problem which is unconditionally stable.

If we want to prove existence of discrete solutions for scheme (81) we will find the following problems. For $\varepsilon>0$, scheme (81) is conditionally solvable for the same reasons explained for scheme (77). In order to solve that problem one may consider

$a\left(\boldsymbol{d}_{h}, \overline{\boldsymbol{d}}_{h}\right)=\left(\nabla \boldsymbol{d}_{h}, \nabla \overline{\boldsymbol{d}}_{h}\right)+\varepsilon^{2}\left(\boldsymbol{d}_{h}, \overline{\boldsymbol{d}}_{h}\right)$

to complete the $H^{1}(\Omega)$ norm. Therefore, the energy estimate remains

$$
\begin{aligned}
& \frac{1}{2}\left\|\boldsymbol{u}_{h}^{n+1}\right\|_{L^{2}(\Omega)}^{2}+\frac{\lambda}{2}\left\|\nabla \boldsymbol{d}_{h}^{n+1}\right\|_{L^{2}(\Omega)}^{2} \\
& +\frac{\lambda \varepsilon^{2}}{4}\left\|\boldsymbol{d}_{h}^{n+1}\right\|_{L^{2}(\Omega)}^{2}+\frac{\lambda \epsilon^{2}}{4}\left\|q_{h}^{n+1}\right\|_{L^{2}(\Omega)}^{2} \\
& +v k \sum_{m=0}^{n}\left\|\nabla \boldsymbol{u}_{h}^{m+1}\right\|_{L^{2}(\Omega)}^{2}
\end{aligned}
$$

$$
\begin{aligned}
& +\frac{\lambda}{\gamma} k \sum_{m=0}^{n}\left\|\delta_{t} \boldsymbol{d}_{h}^{m+1}+\left(\boldsymbol{u}_{h}^{m+1} \cdot \nabla\right) \boldsymbol{d}_{h}^{m+1}\right\|_{L^{2}(\Omega)}^{2} \\
& \leq \frac{1}{2}\left\|\boldsymbol{u}_{h}^{0}\right\|_{L^{2}(\Omega)}^{2}+\frac{\lambda}{2}\left\|\nabla \boldsymbol{d}_{h}^{0}\right\|_{L^{2}(\Omega)}^{2}+\frac{\lambda \varepsilon^{2}}{2}\left\|\boldsymbol{d}_{h}^{0}\right\|_{L^{2}(\Omega)}^{2} \\
& +\frac{\lambda \varepsilon^{2}}{4}\left\|q_{h}^{0}\right\|_{L^{2}(\Omega)}^{2} \quad \text { for all } n .
\end{aligned}
$$

Nevertheless, for $\varepsilon=0$, the existence of the discrete Lagrange multiplier would be established by making use of a discrete version of the inf-sup condition (26) which is far away to be understood. So far, it is known [32] the following discrete version of the inf-sup condition (24)

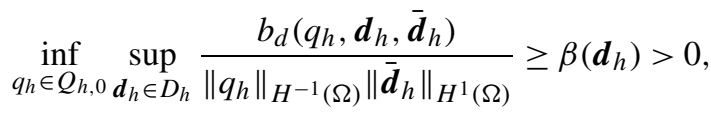

where now the constraint equation is

$b_{d}\left(q_{h}, \boldsymbol{d}_{h}, \overline{\boldsymbol{d}}_{h}\right)=\int_{\Omega} q_{h} I_{Q_{h, 0}}\left(\boldsymbol{d}_{h} \cdot \overline{\boldsymbol{d}}_{h}\right)$

and $Q_{h, 0}=\mathcal{P}_{h}^{1} \cap H_{0}^{1}(\Omega)$. We recall that $I_{Q_{h, 0}}$ is the nodal projection operator into $Q_{h, 0}$. The inf-sup condition (85) has been used in [32] to numerically study the steady-state harmonic map problem whose solution is characterized by a nonlinear saddle-point problem. The discrete solutions are computed by a full Newton linealization which is well-posed if it starts close to a local minimum regular enough. To prove the inf-sup condition (85), the nodal projection operator $I_{Q_{h, 0}}$ played an important role. This projection can be considered without spoiling the stability of schemes (81), (83), and (84). For nodes on the Dirichlet boundaries, it is natural to set the Lagrange multiplier to zero, since the sphere constraint is (hopefully) satisfied by the boundary conditions. However, it is nonsense on Neumann boundaries, since we also need to enforce the sphere constraint there. As was point out in [32], the inf-sup condition (85) is not clear for Neumann boundaries, but numerical experiences showed in [5] indicate that the inf-sup condition also holds in these cases, and subsequently, schemes (81), (83) and (84) are well-posed.

Schemes (81) and (83) enforce the sphere constraint in a discrete sense, as the incompressibility condition for the Navier-Stokes equations. If we consider the modification of the constraint equation (86) proposed in [32] for the projection operator $I_{Q_{h}}$, then one can prove that $\left|\boldsymbol{d}_{h}^{n+1}(\boldsymbol{a})\right|^{2}-$ $\left|\boldsymbol{d}_{h}^{n}(\boldsymbol{a})\right|^{2}+\left|\boldsymbol{d}_{h}^{n+1}(\boldsymbol{a})-\boldsymbol{d}_{h}^{n}(\boldsymbol{a})\right|^{2}=\varepsilon^{2}\left(q_{h}^{n+1}(\boldsymbol{a})-q_{h}^{n}(\boldsymbol{a})\right)$ for scheme (81) and $\varepsilon^{2} q_{h}^{n+1}(\boldsymbol{a})=\left|\boldsymbol{d}_{h}^{n+1}(\boldsymbol{a})\right|^{2}-1$ for scheme (83) at every node $\boldsymbol{a} \in \mathcal{N}_{h}$. To be more precise, we have $q_{h}^{n+1}(\boldsymbol{a})=\varepsilon^{-2}\left(\left|\boldsymbol{d}_{h}^{n+1}(\boldsymbol{a})\right|^{2}-1\right)+\sum_{j=1}^{n}\left|\boldsymbol{d}_{h}^{j+1}(\boldsymbol{a})-\boldsymbol{d}_{h}^{j}(\boldsymbol{a})\right|^{2}$ if we choose $q_{h}^{0}=0$ and $\left|\boldsymbol{d}_{h}^{0}(\boldsymbol{a})\right|=1$ and $q_{h}^{n+1}(\boldsymbol{a})=$ $\varepsilon^{-2}\left(\left|\boldsymbol{d}_{h}^{n+1}(\boldsymbol{a})\right|^{2}-1\right)$ for all nodes $\boldsymbol{a} \in \mathcal{N}_{h}$, respectively. 
Clearly, computing the time derivative of the sphere constraint provides a weaker enforcement at the nodes.

On the other hand, for $\varepsilon=0$, we have that $\left|\boldsymbol{d}_{h}^{n+1}(\boldsymbol{a})\right|^{2}+$ $\sum_{j=1}^{n}\left|\boldsymbol{d}_{h}^{j+1}(\boldsymbol{a})-\boldsymbol{d}_{h}^{j}(\boldsymbol{a})\right|^{2}=1$ and $\left|\boldsymbol{d}_{h}^{n+1}(\boldsymbol{a})\right|^{2}=1$ for schemes (81) and (83), respectively.

It was pointed out in [5] that the closed integration of the constraint trilinear form

$b_{d}\left(q_{h}, \boldsymbol{d}_{h}, \overline{\boldsymbol{d}}_{h}\right) \simeq \sum_{\boldsymbol{a} \in \mathcal{N}_{h}} q(\boldsymbol{a}) \boldsymbol{d}(\boldsymbol{a}) \cdot \overline{\boldsymbol{d}}(\boldsymbol{a}) \int_{\Omega} \boldsymbol{\phi}(\boldsymbol{a})$,

provides the same statement than for the constraint equation (86). Moreover, the $\boldsymbol{d}_{h}-\boldsymbol{q}_{h}$ block matrices in the corresponding linear system are diagonal matrices and hence computationally more appealing.

Iterative algorithms for the implicit schemes (81) and (83) have been developed. For scheme (84), a modified Newton method is designed in order to decouple the computation of the velocity-pressure pair from the director vector and its Lagrange multiplier. For scheme (81) with (82) taking $\varepsilon=0$, a Gauss-Seidel method combined jointly with the projection method due to Alouges $[2,6]$ is also performed. The idea is to consider a Picard linearization of (81) with (82) together with a normalization of the director field approximation.

Let $\left(\boldsymbol{u}_{h}^{n+1, k}, p_{h}^{n+1, k}, \boldsymbol{d}_{h}^{n+1, k}, q_{h}^{n+1, k},\right) \in \boldsymbol{V}_{h} \times P_{h} \times \boldsymbol{D}_{h} \times$ $Q_{h}$ be known.

1. Compute $\left(\tilde{\boldsymbol{d}}_{h}^{n+1, k+1}, q_{h}^{n+1, k+1}\right) \in \boldsymbol{D}_{h} \times Q_{h}$, solution of

$$
\begin{aligned}
& \left(\delta_{t} \tilde{\boldsymbol{d}}_{h}^{n+1, k+1}, \overline{\boldsymbol{d}}_{h}\right)+c\left(\boldsymbol{u}_{h}^{n+1, k}, \tilde{\boldsymbol{d}}_{h}^{n+1, k+1}, \overline{\boldsymbol{d}}_{h}\right) \\
& \quad+\gamma a\left(\tilde{\boldsymbol{d}}_{h}^{n+1, k+1}, \overline{\boldsymbol{d}}_{h}\right) \\
& \quad+\gamma b_{d}\left(q_{h}^{n+1, k+1}, \boldsymbol{d}_{h}^{n+1, k}, \overline{\boldsymbol{d}}_{h}\right)=0, \\
& b_{d}\left(\bar{q}_{h}, \boldsymbol{d}_{h}^{n+1, k}, \tilde{\boldsymbol{d}}_{h}^{n+1, k+1}\right)=\left\langle 1, \bar{q}_{h}\right\rangle .
\end{aligned}
$$

2. Compute $\boldsymbol{d}_{h}^{n+1, k+1} \in \boldsymbol{D}_{h}$ as

$$
\boldsymbol{d}_{h}^{n+1, k+1}(\boldsymbol{a})=\frac{\tilde{\boldsymbol{d}}_{h}^{n+1, k+1}(\boldsymbol{a})}{\mid\left(\tilde{\boldsymbol{d}}_{h}^{n+1, k+1}(\boldsymbol{a}) \mid\right.}
$$

for all $\boldsymbol{a} \in \mathcal{N}_{h}$.

3. Known $\boldsymbol{d}_{h}^{n+1, k+1} \in \boldsymbol{D}_{h}$, compute $\left(\boldsymbol{u}_{h}^{n+1, k+1}, p_{h}^{n+1, k+1}\right) \in$ $V_{h} \times P_{h}$ by a Picard linearization:

$$
\begin{aligned}
& \left(\delta_{t} \boldsymbol{u}_{h}^{n+1, k+1}, \overline{\boldsymbol{u}}_{h}\right)+\widetilde{c}\left(\boldsymbol{u}_{h}^{n+1, k}, \boldsymbol{u}_{h}^{n+1, k+1}, \overline{\boldsymbol{u}}_{h}\right) \\
& \quad+v a\left(\boldsymbol{u}_{h}^{n+1, k+1}, \overline{\boldsymbol{u}}_{h}\right)+b_{u}\left(p_{h}^{n+1, k+1}, \boldsymbol{v}_{h}\right) \\
& \quad+\frac{\lambda}{\gamma} c\left(\overline{\boldsymbol{u}}_{h}, \boldsymbol{d}_{h}^{n+1, k+1}, \partial_{t} \boldsymbol{d}_{h}^{n+1, k+1}\right. \\
& \left.\quad+\left(\boldsymbol{u}_{h}^{n+1, k+1} \cdot \nabla\right) \boldsymbol{d}_{h}^{n+1, k+1}\right)=0, \\
& b_{u}\left(\bar{p}_{h}, \boldsymbol{u}_{h}^{n+1, k+1}\right)=0 .
\end{aligned}
$$

Obviously, $\boldsymbol{d}_{h}^{n+1, k+1}$ satisfies the nonlinear constraint (87) since Step 2 enforces the sphere constraint on the nodes.

With regard to CPU cost, the saddle-point structure needs eight degrees of freedom per node, only beated by schemes (39), (73), and (77) which do not introduced any auxiliary variable. The numerical analysis of schemes (81), (83), and (84) is still open.

\section{Conclusions}

In this work, we have analyzed the existing numerical schemes in the literature to approximate the Ericksen-Leslie equations (3) by means of low-order finite elements. We have distinguished between implicit and semi-implicit methods. Existence of discrete solutions have been detailed for the implicit schemes, as well as their linearization. The convergence of these algorithms is presented by two different ways: compactness and error estimates.

Clearly, schemes (36) and (43) have been designed to satisfy the energy law (17). This is the reason why we think that there is no way to adapt them to hold an energy estimate independent of the penalty parameter $\varepsilon$. The large number of degrees of freedom make them somehow impracticable for large scale simulations.

Probably, scheme (39) is the most efficient algorithm among the linear methods presented herein since it does not compute any extra auxiliary variable. In contrast, there is no mean of finding an energy estimate independent of $\varepsilon$. Schemes (47), (67) and (69), designed for approximating the Ginzburg-Landau equation, need to compute extra variables in order to keep a discrete version of the energy law (7). They introduce between $d$ and $d^{2}$ additional degrees of freedom per node ( $d$ being the space dimension), which implies a too high computationally cost. In particular, although scheme (69) has such an energy estimate, there is no control over the auxiliary unknown $\boldsymbol{w}=-\Delta \boldsymbol{d}$ independent of $\varepsilon$, which could clearly deteriorate the resulting linear system. Another important drawback of schemes (47) and (69) is the fact that they are conditionally stable. The relation (45) is quite restrictive, in the sense that one needs a time step small for moderate values of $\varepsilon$ if $\varepsilon=\mathcal{O}(h)$. It is interesting to note that scheme (67) is unconditionally stable, but it requires the extra relation (46) to pass to the limit towards measure-valued solutions, as was pointed out in [29].

One of the main advantages of using the saddle-point approach is that it allows to approximate numerically both the Ericksen-Leslie problem and the Ginzburg-Landau problem by means of the same numerical approximation. Moreover, it allows to take $\varepsilon$ arbitrary small (or even zero) in comparison with the previous algorithms, since the condition number is independent of $\varepsilon$. However, the proof of the inf-sup condition that would allow to prove the well-posedness of 
the algorithm is still open. Numerical tests have shown an $\left(H^{1}\right)^{\prime}$ stability in [5]. We would also mention that the nonlinearity in the equation for the director vector is quadratic while for other alternatives it is cubic.

The best choice to approximate the Eriscksen-Leslie equations in terms of the number of degrees of freedom is scheme (77), since it does not introduce any extra variable. Nevertheless, the convergence is not clear even for twodimensional problems due to the fact the cubic nonlinearity involves second derivatives.

\section{References}

1. Adams RA (1975) Sobolev spaces. Academic Press, New York

2. Alouges F (1997) A new algorithm for computing liquid crystal stable configurations: the harmonic mapping case. SIAM J Numer Anal 34(5):1708-1726

3. Azérad P, Guillén-González F (2001) Mathematical justification of the hidrostatic approximation in the primitive equations of geophysical fluid dynamics. SIAM J Math Anal 33(4):847-859

4. Badia S, Codina R (2009) On a multiscale approach to the transient Stokes problem: dynamic subscales and anisotropic spacetime discretization. Appl Math Comput 207:415-433

5. Badia S, Guillén-González F, Gutiérrez-Santacreu JV (2011) Finite element approximation of nematic liquid crystal flows using a saddle-point structure. J Comput Phys 230(4):1686-1706

6. Bartels S (2005) Stability and convergence of finite-element approximation schemes for harmonic maps. SIAM J Numer Anal 43(1):220-238

7. Bartels S, Prohl A (2007) Constraint preserving implicit finite element discretization of harmonic map heat flow into spheres. Math Comput 76:1847-1859

8. Becker R, Feng X, Prohl A (2008) Finite element approximations of the Ericksen-Leslie model for nematic liquid crystal flow. SIAM J Numer Anal 46(4):1704-1731

9. Brezzi F, Fortin M (1991) Mixed and hybrid finite element methods. Computational mathematics, vol 15. Springer, Berlin

10. Chen YM (1989) The weak solutions to the evolution problems of harmonic maps. Math Z 201(1):69-74

11. Cuvelie C, Segal A, van Steenhoven A (1986) Finite element methods and Navier-Stokes equations. Reidel, Dordrecht

12. Dauge M (1989) Stationary Stokes and Navier-Stokes systems on two or three-dimensional domains with corners. SIAM J Math Anal 20(1):74-97

13. Dauge M (1989) Problèmes mixtes pour le laplacien dans des domaines polyédraux courbes. (French. English summary). C R Acad Sci Paris Sér I Math 309(8):553-558

14. Dauge M (1992) Neumann and mixed problems on curvilinear polyhedra. Integral Equ Oper Theory 15, 227-261

15. Douglas JJ, Dupont T, Wahlbin L (1974/75) The stability in $L^{q}$ of the $L^{2}$-projection into finite element function spaces. Numer Math 23:193-197

16. de Gennes PG (1974) The physics of liquid crystals. Oxford University Press, London

17. de Gennes PG, Prost J (1995) The physics of liquid crystals. Clarendon Press, Oxford

18. Ericksen J (1961) Conservation laws for liquid crystals. Trans Soc Rheol 5:22-34

19. Ericksen J (1987) Continuum theory of nematic liquid crystals. Res Mech 21, 381-392

20. Ern A, Guermond JL (2004) Theory and practice of finite elements. Springer, Berlin
21. Frank FC (1958) On the theory of liquid crystals. Discuss Faraday Soc 25:19-28

22. Feng X, Prohl A (2004) Analysis of a fully discrete finite element method for the phase field model and approximation of its sharp interface limits. Math Comput 73(246):541-567

23. Girault V, Guillén-González F (2011) Mixed formulation, approximation and decoupling algorithm for a nematic liquid crystals model. Math Comput 80:781-819

24. Girault V, Lions JL (2001) Two-grid finite-element schemes for the transient Navier-Stokes problem. Modél Math Anal Numér 35:945-980

25. Girault V, Nochetto N, Scott R (2004) Estimates of the finite element Stokes projection in $W^{1, \infty}$. C R Math Acad Sci Paris 338(12):957-996

26. Girault V, Raviart PA (1986) Finite element methods for NavierStokes equations: theory and algorithms. Springer, Berlin

27. Guillén-González F, Gutiérrez-Santacreu JV (2008) Unconditional stability and convergence of a fully discrete scheme for $2 D$ viscous fluids models with mass diffusion. Math Comput 77(63): 1495-1524

28. Guillén-González F, Gutiérrez-Santacreu JV (2008) Conditional stability and convergence of a fully discrete scheme for $3 D$ Navier-Stokes equations with mass diffusion. SIAM J Numer Anal 46(5):2276-2308

29. Guillén-González F, Gutiérrez-Santacreu JV (2011) A linear mixed finite element scheme for a nematic Ericksen-Leslie liquid crystal model. Submitted

30. Grisvard P (1985) Elliptic problems in nonsmooth domains. Pitman monographs and studies in mathematics, vol 24. Pitman, Boston

31. Heywood JG, Rannacher R (1982) Finite element approximation of the nonstationary Navier-Stokes problem. I. Regularity of solutions and second-order error estimates for spatial discretization. SIAM J Numer Anal 19(2), 275-311

32. Hu Q, Tai X-C, Winther R (2009) A saddle-point approach to the computation of harmonic maps. SIAM J Numer Anal 47(2), 1500 1523

33. Hughes TJR, Franca LP, Balestra M (1986) A new finite element formulation for computational fluid dynamics: V. Circumventing the Babuŝka-Brezzi condition: a stable Petrov-Galerkin formulation for the Stokes problem accommodating equal-order interpolations. Comput Methods Appl Mech Eng 59:85-99

34. Kellogg RB, Osborn JE (1976) A regularity for the Stokes problem in a convex polygon. J Funct Anal 21:397-431

35. Leslie F (1979) Theory of flow phenomenum in liquid crystals. In: Brown W (ed) The theory of liquid crystals, vol 4, pp 1-81. Academic Press, New York

36. Leslie F (1968) Some constitutive equations for liquid crystals. Arch Ration Mech Anal 28:265-283

37. Lin FH (1989) Nonlinear theory of defects in nematic liquid crystals: phase transition and flow phenomena. Commun Pure Appl Math 42:789-814

38. Lin FH, Liu C (1995) Non-parabolic dissipative systems modelling the flow of liquid crystals. Commun Pure Appl Math 48:501-537

39. Lin FH, Liu C (2000) Existence of solutions for the EricksenLeslie system. Arch Ration Mech Anal 154, 135-156

40. Lin P, Liu C (2006) Simulations of singularity dynamics in liquid crystal flows: A $C^{0}$ finite element approach. J Comput Phys 215:348-362

41. Lin P, Liu C, Zhang H (2007) An energy law preserving $C^{0}$ finite element scheme for simulating the kinematic effects in liquid crystal dynamics. J Comput Phys 227(2):1411-1427

42. Lions JL (1978) On some questions in boundary value problems of mathematics physics. In: Contempory developments in continuum mechanics and partial differential equations. North-Holland math stud, vol 30, pp 283-346. North-Holland, Amsterdam 
43. Lions JL, Magenes E (1972) Nonhomogeneous boundary value problems and applications, vol I. Die Grundlehren der Mathematischen Wissenschaften, vol 181. Springer, New York. Translated from the French by P Kenneth

44. Liu C, Walkington NJ (2000) Approximation of liquid crystal flows. SIAM J Numer Anal 37(3):725-741

45. Liu C, Walkington NJ (2002) Mixed methods for the approximation of liquid crystal flows. Modél Math Anal Numér 36(2):205222

46. Liu C, Walkington NJ (2007) Convergence of numerical approximations of the incompressible Navier-Stokes equations with variable density and viscosity. SIAM J Numer Anal 45(3):1287-1304

47. Malek J, Necas J, Rokyta M, Ruzicka M (1996) Weak and measure-valued solutions to evolutionary PDEs. Chapman \& Hall, New York

48. Necas J (1967) Les Méthodes directes en théorie des équations elliptiques. Masson, Paris

49. Oseen C (1933) Theory of liquid crystals. Trans Faraday Soc 29:883-899
50. Protter M, Weinberger H (1967) Maximum principles in differential equations. Prentice-Hall, Englewood Cliffs

51. Savaré G (1998) Regularity results for elliptic equations in Lipschitz domains. J Funct Anal 152(1):176-201

52. Scott LR, Zhang S (1990) Finite element interpolation of nonsmooth functions satisfying boundary conditions. Math Comput $54: 483-493$

53. Shatah J (1988) Weak solutions and development of singularities in $S U$ (2) $\sigma$-model. CPAM 41, 459-469

54. Simon J (1987) Compact sets in the space $L^{p}(0, T ; B)$. Ann Mat Pura Appl 146:65-97

55. Temam R (1984) Navier-Stokes equations. North-Holland, Amsterdam

56. Raviart PA, Thomas JM (1977) A mixed-finite element method for second order elliptic problems. In: Mathematical aspects of the finite element method. Lecture notes in mathematics. Springer, New York 